\title{
HPLC method development for the assessment of tryptophan metabolism and its application in a complete Freund's adjuvant model of orofacial pain
}

Cseh Edina Katalin

Ph.D. Thesis

Szeged

2020 


\section{HPLC method development for the assessment of tryptophan metabolism and its application in a complete Freund's adjuvant model of orofacial pain}

Ph.D. Thesis

Cseh Edina Katalin

Department of Neurology

Faculty of Medicine

Albert Szent-Györgyi Clinical Center

Doctoral School of Clinical Medicine

University of Szeged

Supervisor: Dénes Zádori M.D., Ph.D.

Szeged

2020 
Original publications directly related to the Ph.D. thesis:

I. Cseh E.K., Veres G., Szentirmai M., Nánási N., Szatmári I., Fülöp F., Vécsei L., Zádori D. HPLC method for the assessment of tryptophan metabolism utilizing separate internal standard for each detector. Anal. Biochem. 2019, 574:7 (original paper, IF: 2.877)

II. Cseh E.K., Veres G., Körtési T., Polyák H., Nánási N., Tajti J., Klivényi P., Vécsei L., Zádori D. Neurotransmitter and tryptophan metabolite concentration changes in the Complete Freund's adjuvant model of orofacial pain. J. Head. Pain. 2020, 21:35 (original paper, $\operatorname{IF}(\mathbf{2 0 1 9})$ : 4.797 )

Cumulative impact factor of the publications directly related to the thesis: $\mathbf{7 . 6 7 4}$ 


\section{Publications not directly related to the Ph.D. thesis:}

I. Cseh E.K, Nánási N., Veres G., Klivényi P., Danics K., Vécsei L., Kovács G.G., Zádori D. The assessment of concentrations of certain tryptophan metabolites in CreutzfeldtJakob disease. Proceeding of the 23rd International Symposium on Analytical and Environmental Problems. 2017. ISBN 978-963-306-563-1 (conference proceeding).

II. Nánási N., Cseh E.K., Szentirmai M., Veres G., Klivényi P., Vécsei L., Zádori D. Development and validation of high performance liquid chromatography methods for vitamin e measurements. Proceeding of the 23rd International Symposium on Analytical and Environmental Problems. 2017. ISBN 978-963-306-563-1 (conference proceeding).

III. Nánási N., Veres G., Cseh E.K., Szentirmai M., Martos D., Sümegi E., Hadady L., Klivényi P., Vécsei L., Zádori D. The detection of age-, gender-, and region-specific changes in mouse brain tocopherol levels via the application of different validated HPLC methods. Neurochem. Res. 2018, 43:2081 (original paper, IF: 2.782)

IV. Szalardy L., Molnar F.M., Zadori D., Cseh E.K., Veres G., Kovacs G.G., Vecsei L., Klivenyi P. Non-motor behavioral alterations of PGC1 $\alpha$-deficient mice - a peculiar phenotype with slight male preponderance and no apparent progression. Front. Behav. Neurosci. 2018, 12:180 (original paper IF: 3.104)

V. Cseh E.K., Veres G., Danics K., Szalárdy L., Nánási N., Klivényi P., Vécsei L., Zádori D. Additional value of tau protein measurement in the diagnosis of Creutzfeldt-Jakob disease. Ideggy. Sz. 2019, 72: 39 (original paper IF: 0.337)

VI. Nánási N., Hadady L., Cseh E., Veres G., Klivényi P., Vécsei L., Zádori D. Development and validation of high performance liquid chromatography method for the measurements of biogenic amines. Proceeding of the 24th International Symposium on Analytical and Environmental Problems. 2018. ISBN 978-963-306-623-2 (conference proceeding).

VII. Herédi J., Cseh E.K., Magyariné B.A, Veres G., Zádori D., Toldi J., Kis Zs., Vécsei L., Ono E., Gellért L. Investigating KYNA production and kynurenergic manipulation on 
acute mouse brain slice preparations. Brain Res. Bull. 2019, 146:185 (original paper IF: $3.370)$

VIII. Tömösi F., Kecskeméti G., Cseh E.K., Szabó E., Rajda C., Kormány R., Szabó Z., Vécsei L., Janáky T. A validated LC-MS method for tryptophan metabolites: Application in the diagnosis of multiple sclerosis. J. Pharm. Biomed. Anal. 2020.185:113246 (original paper, $\operatorname{IF}(\mathbf{2 0 1 9})$ : 3.209)

IX. Zádor F., Nagy-Grócz G., Dvorácskó S., Bohár Z., Cseh E.K., Zádori D., Párdutz Á., Szücs E., Tömböly C., Borsodi A., Benyhe S., Vécsei L. Long-term sytemic administration of kynurenic acid brain region specifically elevates the abundance of functional CB1 receptors in rats. Neurochem. Int. 2020 (IF(2019): 3.881).

Cumulative impact factor of publications not directly related to the thesis: $\mathbf{1 6 . 6 8 3}$

Total impact factor: $\mathbf{2 4 . 3 5 7}$ 


\section{Table of contents}

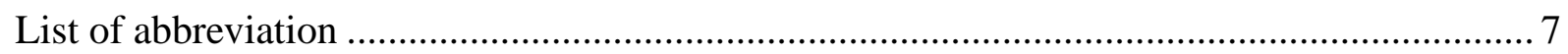

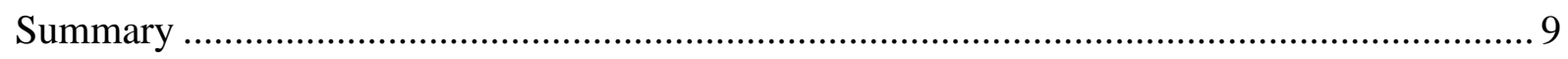

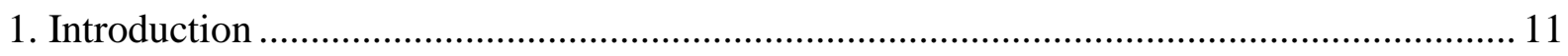

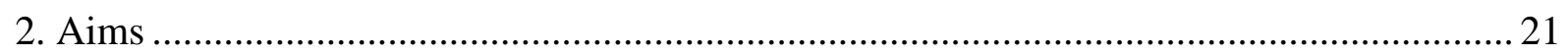

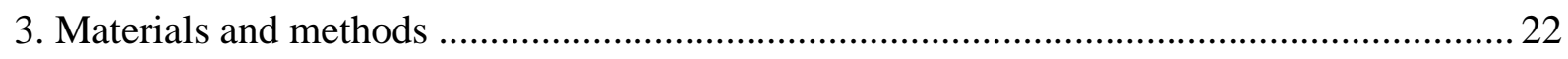

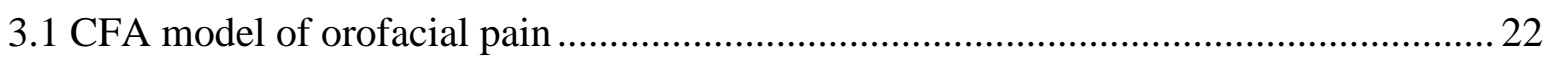

3.2 Quantification of TRP, 5-HT, KYN and KYNA from different biological samples ..... 24

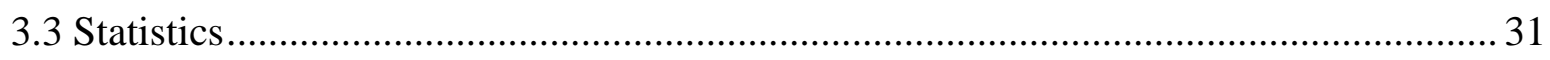

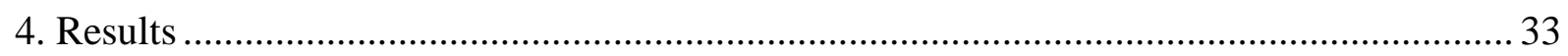

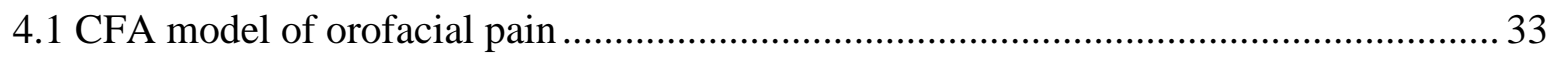

4.2 Quantification of TRP, 5-HT, KYN and KYNA from different biological samples ..... 38

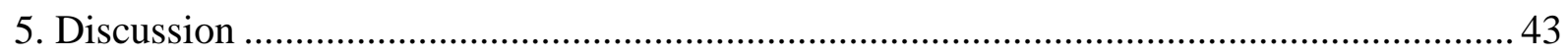

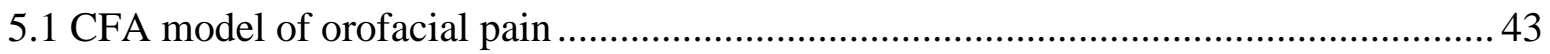

5.2 Quantification of TRP, 5-HT, KYN and KYNA from different biological samples ..... 45

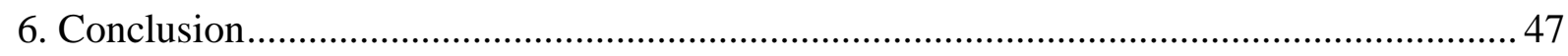

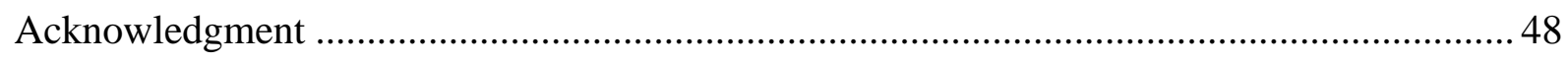

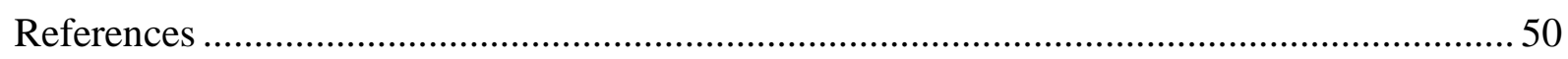




\section{List of abbreviation}

3HANA - 3-hydroxy anthranilic acid

3NLT - 3-nitro-L-tyrosine

3MP - 3-mercaptopropionic acid

5-HT - serotonin

ACC - anterior cingulate cortex

$\mathrm{ACN}$ - acetonitrile

$\mathrm{AD}$ - Alzheimer's disease

AMPA - $\alpha$-amino-3-hydroxy-5-methyl-4-isoxazolepropionic acid

BBB - blood brain barrier

C1-C2 - upper part of cervical spine

CGRP - calcitonin gene-related peptide

CNS - central nervous system

$\mathrm{CO}$ - control groups

CSF - cerebrospinal fluid

$\mathrm{CV}$ - coefficient of variation

DALY - disability-adjusted life years

DRG - dorsal root ganglion,

FLD - fluorescent detection

GABA $-\gamma$-aminobutyric acid

Glu - glutamate

HCA - 4-hydroxyquinolizone carboxylic acid

HPLC - high performance liquid chromatography

ICH - International Conference on Harmonization

IS - internal standard

KAT - kynurenine aminotransferase

$\mathrm{KMO}$ - kynurenine 3-monooxygenase

$\mathrm{KP}$ - kynurenine pathway

KYN - kynurenine 
KYNA - kynurenic acid

LC - locus coeruleus

LOD - limit of detection

LOQ - lower limit of quantification

$\mathrm{Na}_{2} \mathrm{EDTA}$ - disodium ethylenediaminetetraacetate

NMDA - N-methyl-D-aspartate

$\mathrm{NRM}$-nucleus raphe magnus

OPA - o-phthaldialdehyde

PACAP - pituitary adenylate cyclase activating polypeptide

PAG - periaqueductal grey matter,

PCA - perchloric acid

PD - Parkinson's disease

PfCx - prefrontal cortex

QC - quality controls

$\mathrm{RPM}$ - rotation per minute

S1/S2 - primary and secondary somatosensory cortex

ssCX - somatosensory cortex

TCC - trigeminocervical complex

TNC - trigeminal nucleus caudalis

$\mathrm{TG}$ - trigeminal ganglion

TRP - tryptophan

UVD - UV detector

V1 - primary visual cortex

WS - working solution

$\mathrm{ZnAc}$ - zinc acetate 


\section{Summary}

The pathomechanism of orofacial pain and headache disorders, including migraine is complex and need further elucidation. Nevertheless, the activation and sensitization of the trigeminovascular system has especially important role in the development of symptoms. For better understanding of the pathomechanism of these disorders, animal models with the activation of nociceptive pathways of the trigeminovascular system are used. Complete Freund's adjuvant (CFA)-induced orofacial pain model serves for the induction of peripheral inflammation. Therefore, the investigation of the neurochemical profile of this model could provide meaningful information regarding pain processing.

In the field of neuroscience, the information obtained via the measurement of biomarkers may aid the diagnosis, prevention and treatment of different neurological disorders. The main purpose of the bioanalytical assessments is to reveal whether there are changes on molecular levels during the development, course and treatment of a disease, either from a clinical or preclinical point of view.

Our aim was to investigate the concentration changes of some biomarkers, including glutamate (Glu), $\gamma$-aminobutyric acid (GABA), and serotonin, and in light of its influence on glutamatergic neurotransmission, we further expanded the investigation of the neurochemical profile with the measurement of the level of kynurenic acid (KYNA) and its precursors in the kynurenine $(\mathrm{KYN})$ pathway $(\mathrm{KP})$ of tryptophan metabolism. Furthermore, due to the importance of the KP, we also aimed at the method optimization and validation on six different biological matrices, including human plasma and cerebrospinal fluid (CSF), mouse brain and plasma, and rat central nervous system (CNS) and plasma.

The CFA model consisted of the CFA ( $1 \mathrm{mg} / \mathrm{ml}, 50 \mu \mathrm{l} /$ animal $)$ injection into the right whisker pad of male Sprague-Dawley rats. The samples were collected 24 and $48 \mathrm{~h}$ after injection, whereas the control group rats, injected with saline, were processed at $24 \mathrm{~h}$ after injection. Two important brain regions were chosen to determine the concentration of the above-mentioned metabolites in the trigeminal nucleus caudalis (TNC) and somatosensory cortex (ssCX), as both have an important role in the pain processing. We applied high-performance liquid chromatography (HPLC) coupled with UV and fluorescence detection, using separate internal standards for each detector: 3-nitro-L-tyrosine and the newly utilized 4-hydroxyquinazoline-2carboxylic, respectively, to determine the concentration changes of the metabolites. For further 
method validation process, carried out on the different biological matrices, the same HPLC method was used in each case, with slight modifications.

In the CFA model, our results demonstrated that $24 \mathrm{~h}$ after CFA treatment, the level of Glu, KYNA and that of its precursor, KYN, were still elevated in the TNC, all diminishing by $48 \mathrm{~h}$. In the ssCX, significant concentration increases of KYNA and serotonin were found. Regarding the assessment of some TRP metabolites, the method was successfully utilized for measurements from human plasma and CSF, mouse brain and plasma, and rat CNS and plasma. Regarding murine CNS samples, serotonin was successfully measured as well in one single run with TRP, KYN and KYNA. During the method validation, good intra- and inter-day precision values were obtained with coefficient of variation $<5 \%$, and bias $<6.5 \%$ (except the serotonin levels in murine CNS samples), respectively. The recoveries varied between $79.6 \%$ and $116 \%$, with all results being in line with the official guidelines.

The results from the CFA animal model confirm the dominant role of Glu in early pain processing and a compensatory elevation of KYNA with anti-glutamatergic properties. Furthermore, the current findings draw attention to the limited time interval where medications can target the glutamatergic pathways. During the validation process of the CNS samples, the high bias values of serotonin draws attention to the necessity of brain homogenization right before the measurement in line with our currently applied laboratory practice. The optimized and validated methods on six different biological matrices yield opportunities for the assessment of concentration changes in the TRP metabolism from a wide range samples related to neuroscience research; therefore, they may be utilized well in future clinical and preclinical studies. 


\section{Introduction}

Neurological disorders are recognized as the leading cause of disability and second leading cause of death worldwide (GBD 2015 DALYs and HALE Collaborators, 2016). Therefore, their contribution to the overall burden from all health conditions is increasing (GBD 2016 Neurology Collaborators, 2019). These diseases, including headache disorders (e.g. migraine, tension-type headache and medication overuse headache), multiple sclerosis, epilepsy or neurodegenerative conditions, such as Alzheimer's disease (AD) or Parkinson's disease (PD) represent three percent of the worldwide burden of disease (GBD 2016 Neurology Collaborators, 2019). Although it may seem to be a small rate, however, regarding disabilityadjusted life years (DALYs) the numbers are increasing: migraine, stroke, epilepsy and dementia rank in the top 50 causes of DALYs (Murray et al., 2012). Moreover, from all neurological disorders, headache disorders have a considerably high prevalence, with a $46 \%$ of population suffering from headache in general for 1-year prevalence and $64 \%$ for lifetime prevalence, from which the majority are diagnosed with primary headache. The prevalence of this type is very high, with $14.4 \%$ from migraine, $26.1 \%$ from tension-type headache (GBD 2016 Headache Collaborators, 2018) and 3\% to 5\% from chronic daily headache (Silberstein, 2005). Secondary headaches are resulting from the traction or inflammation of pain-sensitive structures (Rizzoli \& Mullally, 2018). In 2011, a report presented by World Health Organization (World Health Organization and Lifting The Burden, 2011) described that only 7\% of the headaches are diagnosed as a secondary headache. In neurological disorders, including headache disorders and neuropathies resulting from primary or secondary etiologies, the prevalence of pain, both central or peripheral, is very high (Borsook, 2012). Furthermore, patients suffering from neurodegenerative disorders complain about painful symptoms as well with a prevalence varying from 38 to $75 \%$ in $\mathrm{AD}$ and $40 \%$ to $86 \%$ in PD (de Tommaso et al., 2016). Although in AD the pain features have not been defined (Zwakhalen et al., 2009), in PD and amyotrophic lateral sclerosis a prevalent nonneuropathic origin of pain has been described (de Tommaso et al., 2016).

There are three neuronal levels involved in the pain signaling process (Figure 1). The first neurons, which have special receptors called nociceptors, have their cell bodies within the dorsal root or the sensory ganglia of the cranial nerves (e.g. trigeminal ganglia (TG)) and they are activated through various stimuli (chemical, thermal and mechanical), both external and internal (Garland, 2012). 


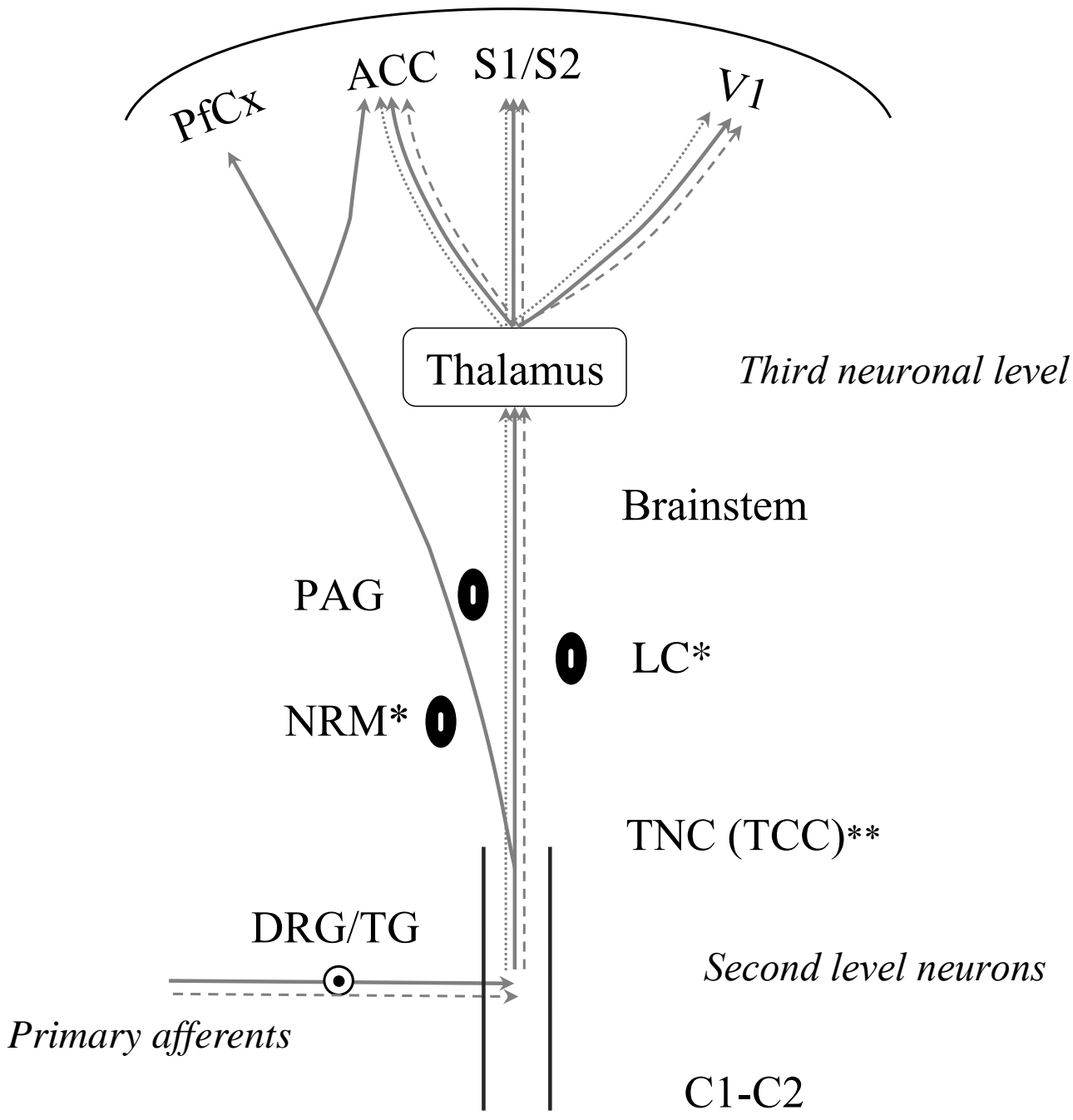

Figure 1. Schematic representation of relevant pain related projections of trigeminal system, including the glutamatergic and serotonergic projections. Straight arrows (-) represent the migraine and head pain relevant projections (Tajti et al., 2011; Goadsby et al., 2017), dashed (--) arrows the glutamatergic projections (Noseda \& Burstein, 2013; Goadsby et al., 2017), the dotted arrows ( $\cdots$ ) the serotonergic projections (Deen et al., 2017). *Both NRM and LC are implicated in the serotonergic and noradrenergic projections. ${ }^{*}$ Cross-projection are described in the TNC (TCC) area. ACC anterior cingulate cortex, $C 1-C 2$ upper part of cervical spine, $D R G$ dorsal root ganglion, $L C$ locus coeruleus, $N R M$ nucleus raphe magnus, $P A G$ periaqueductal grey matter, $P f C x$ prefrontal cortex, $S 1 / S 2$ primary and secondary somatosensory cortex, $T C C$ trigeminocervical complex, $T G$ trigeminal ganglion, $T N C$ trigeminal nucleus caudalis, $V 1$ primary visual cortex. The schematic representation is based on the work of Tajti and his colleagues (Tajti et al., 2011).

The signals are further conveyed to the dorsal horn of the spinal cord or to the trigeminal nucleus caudalis (TNC), recently also called as trigeminocervical complex (TCC), leading to the release 
of the neurotransmitters such as glutamate (Glu), calcitonin gene related peptide (CGRP), substance $\mathrm{P}$, neurokinin A and pituitary adenylate cyclase activating peptide (PACAP). The corelease of Glu and CGRP is controlled by calcium influx via the P/Q-type channels (Xiao et al., $2008 b$ ), the latter leading to the activation of CGRP receptors, further evoking the release of Glu and Substance P. Moreover, CGRP receptors were identified presynaptically in the dorsal spinal horn on nerve terminals of glutaminergic neurons and their activation sensitizes the $\alpha$ amino-3-hydroxy-5-methyl-4-isoxazole propionic acid (AMPA) and N-methyl-D-aspartate (NMDA) receptors (Benarroch, 2011), which may promote the release of Glu at this level too (Marvizón et al., 2007). The release of Glu subsequently increases NMDA receptor expression (Newcomer et al., 2000), further promoting and maintaining a sensitized state (Latremoliere \& Woolf, 2009). The ionotropic Glu receptors, namely the NMDA, AMPA and kainate receptors, along with the metabotropic Glu receptors, are localized within various parts of the TS and TNC as well (Tallaksen-Greene et al., 1992). High densities of the mentioned ionotropic receptors can be found in the superficial laminae I and II of the Sp5 subdivision of the TNC (Furuyama et al., 1993). NMDA receptor mRNA was found in the trigeminal ganglion cells as well (Watanabe et al., 1981 ). Substance P transmits nociceptive signals via primary afferent fibers to the spinal cord and brainstem second level neurons (Zieglgänsberger, 2019). CGRP and PACAP show similar pattern in both TG and TNC during the activation of TS (Aczél et al., 2018; Edvinsson et al., 2018; Körtési et al., 2019), and PACAP even shows a more obvious increasing trend during repetitive stimuli compared to CGRP (Edvinsson et al., 2018).

The axons of second level neurons, the cell bodies of which are generally located in the spinal cord or brainstem, ascend further to the thalamus, from where the third level neurons project to the primary somatosensory cortex (Bolay \& Moskowitz, 2002; Garland, 2012). At this level, there are several other neurotransmitters and neuromodulators, which are able to modulate the presented process of pain sensation, e.g. via the activation of $\gamma$-aminobutyric acid (GABA)ergic or glycinergic inhibitory neurons. The major inhibitory neurotransmitter, GABA is involved in the augmentation of the descending inhibition of spinal nociceptive neurons (Jasmin et al., 2003). GABA may be capable to restore the impaired inhibitory-excitatory balance (Wu \& Sun, 2015) and thereby has a role in the modulation of pain perception as well (Enna \& McCarson, 2006). Not only GABA, but both serotonergic and noradrenergic axons, originating from different brainstem regions, such as the nucleus raphe magnus (NRM) or locus coeruleus 
(LC), are involved in the descending inhibition that project to the spinal cord and brainstem (Beitz, 1982; Braz et al., 2009; Michael-Titus et al., 2010).

As partially mentioned above, during the process of orofacial pain and headache, the major mechanism is the activation and sensitization of the trigeminovascular system (TS) (TallaksenGreene et al., 1992; Sahara et al., 1997; Quartu et al., 2002; Pietrobon \& Moskowitz, 2013; Noseda \& Burstein, 2013; Brennan \& Pietrobon, 2018). Continuous activation of peripheral trigeminal afferents leads to peripheral sensitization (throbbing feature of headache and exercise and physical activity-induced headache) (Burstein et al., 1998), resulting in primary hyperalgesia, i.e., increased perception of the painful stimuli. This may result in the sensitization of the second and ultimately that of the third level neurons, a phenomenon called central sensitization (Goadsby et al., 2017), when non-painful stimuli are perceived as painful (allodynia - cephalic or extracephalic) and secondary hyperalgesia evolves. It was also demonstrated that if the central sensitization develops, the treatment becomes less effective (Burstein et al., 2004).

Taken together, Glu and both its ionotropic and metabotropic receptors have pivotal role in the pathophysiology of headache and pain (Soliman et al., 2005). The importance of the NMDA receptors culminates at the point where their activation becomes one of the most important steps in initiating and maintaining the central sensitization (Latremoliere \& Woolf, 2009). The increase of Glu level is demonstrated in different animal models of headache and pain (Oshinsky \& Luo, 2006). The stimulation of the trigeminal nerve resulted in elevated Glu levels in the spinal part of the TNC (Oshinsky \& Luo, 2006). The peripheral application of Glu to deep craniofacial tissue proved to activate and sensitize nociceptive afferents and neurons in the upper cervical cord (Lam et al., 2009). Data from human studies, regarding head pain, consistently showed elevated Glu levels in the cerebrospinal fluid (CSF) samples of patients with chronic migraine (Peres et al., 2004), or migraine with and without aura (Martínez et al., 1993), whereas in plasma samples the results were not consistent across studies (Ferrari et al., 1990; Cananzi et al., 1995; Campos et al., 2013). Nevertheless, the available data indicate the presence of hyperexcitability in headache-related disorders (Vécsei et al., 2015). The importance of NMDA receptors in pain processing, including migraine, is underlined by the fact that ketamine, as one of its antagonist, showed promising therapeutic effects in patients with severe or long lasting migraine with aura (Afridi et al., 2013). Substances, such as 
tezampanel, which can act at AMPA and kainate receptors, has been proved to have promising beneficial effects on migraine as well (Sang et al., 2004).

GABA receptor agonists as well as inhibitors of GABA uptake and metabolism display significant antinociceptive activity in animal models of different pain conditions (Levy \& Proudfit, 1977; Kendall et al., 1982; Malan et al., 2002; Polgár et al., 2003; Sands et al., 2004). The serotoninergic system is involved in the primary headaches, including migraine, in many ways. During migraine attack the concentration of the main metabolite of 5-HT, 5hydroxyindole acetic acid, increases in the urine (Sicuteri et al., 1961), whereas platelet 5-HT concentration decreases (Anthony et al., 1967). The reserpine- and fenfluramine-induced 5-HT release might lead to migraine attacks (Silberstein, 1994), whereas when applied intravenously, 5-HT injection will block these attacks (Kimball et al., 1960).

Kynurenic acid (KYNA), a product of the kynurenine (KYN) pathway (KP) of tryptophan (TRP) metabolism (Figure 2), is also capable of influencing the glutamatergic neurotransmission in a complex way (Zádori et al., 2011b). It acts as a competitive antagonist at the NMDA receptor (Kessler et al., 1989) and has weak antagonistic effects at the AMPA and kainate receptors as well (Birch et al., 1988). The KP of the essential amino acid TRP accounts for $95 \%$ of its degradation, whereas the remaining $5 \%$ is degraded through the 5-HT pathway. With its biologically active metabolites, including the above-mentioned KYNA with mostly neuroprotective properties (Kessler et al., 1989; Grant et al., 2009; Vécsei et al., 2013), the KP of TRP metabolism became of interest in different research field (Schwarcz et al., 2012; Vécsei et al., 2013). The antinociceptive properties of KYNA has been proved in different animal models of pain: in the study of chronic osteoarthritis-like joint pain (Tuboly et al., 2015), in carrageenan-induced thermal hyperalgesia (Kekesi et al., 2002) or in a model of inflamed joint (Mecs et al., 2009). Furthermore, some of the developed analogs also displayed promising results in different animal models of headache, including the formalin model of trigeminal pain as well (Knyihar-Csillik et al., 2008; Vámos et al., 2010; Park et al., 2011; Fejes-Szabó et al., 2014; Veres et al., 2017). Furthermore, TRP, KYN and KYNA have been related to migraine and other headache disorders (Curto et al., 2015a, 2015b), i.e., significant reductions in the serum levels of KYN and KYNA were demonstrated, whereas increased concentration levels of TRP were found in migraine and cluster headache. 


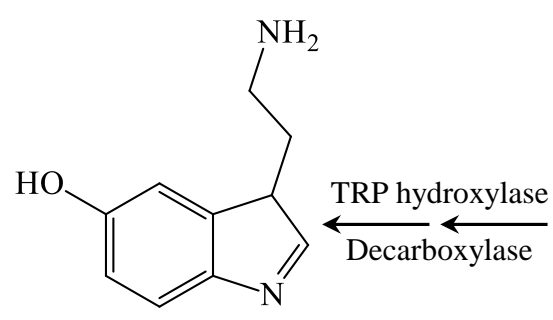

5-hydroxytryptamine (Serotonin)<smiles>N[C@@H](Cc1c[nH]c2ccccc12)C(=O)O</smiles>

$L$-tryptophan

IDO, TDO
L-kynurenine

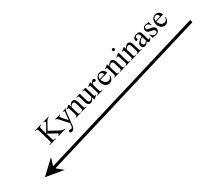

Anthranilic

acid

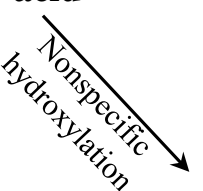

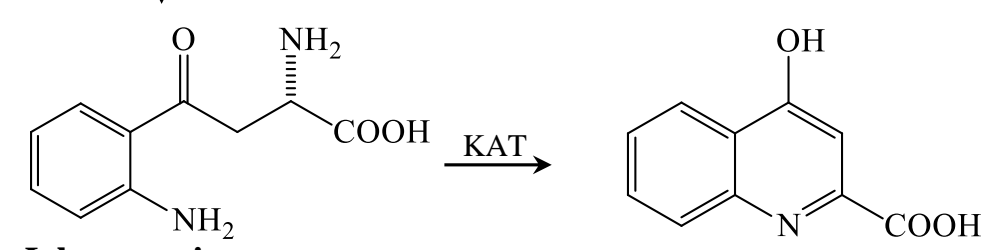

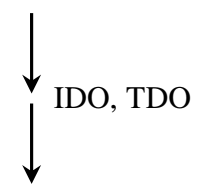

Kynurenic acid

3-hydroxyanthranilic

\section{3-hydroxy- $\boldsymbol{L}$ - $\stackrel{\mathrm{KAT}}{\longrightarrow}$ Xanthurenic kynurenine \\ acid}

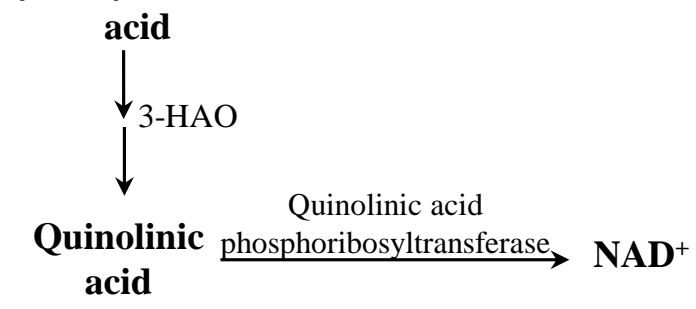

Figure 2. The partial kynurenine and serotonin pathway of the tryptophan metabolism. 3-HAO

3-hydroxyanthranilate oxidase, KAT kynurenine aminotransferase, KMO kynurenine

3-monooxygenase, IDO indoleamine 2,3-dioxygenase, $N A D$ nicotinamide adenine dinucleotide, TDO tryptophan 2,3-dioxygenase.

The concentration changes of neurotransmitters and the above-mentioned neuropeptides, including CGRP and PACAP has been studied deeply in experimental models of pain including that of migraine (Kendall et al., 1982; Ferrari et al., 1990; Cananzi et al., 1995; Polgár et al., 2003; Sands et al., 2004; Oshinsky \& Luo, 2006; Vámos et al., 2010; Markovics et al., 2012; Tuka et al., 2012, 2013; Syed et al., 2012; Campos et al., 2013; Körtési et al., 2019). However, 
no studies have been carried out aiming at finding a shift point between the concentration changes of small molecule neurotransmitters and neuropeptides, which data could yield substantial information for the selection between different therapeutic paradigms regarding different phases of disorders with the activation of the TS.

On the whole the measurement of these compounds of interest, which may be designated as biomarkers, is crucial, as they may have a potential role in the development, course, treatment and diagnosis of the diseases. Biomarkers, measured accurately and reproducibly from different biological fluids, including blood plasma or serum, CSF or tissues can predict the progression and outcome of a disease, and moreover, can be used to track any effect of applied and potential novel drugs either at cellular or molecular level (WHO, 2001; Strimbu \& Tavel, 2010). Biomarkers represent a very important part of the neuroscience research, which aims to assess the nervous system under physiological and pathological conditions.

Regarding the measurement of the main excitatory neurotransmitter, Glu, it is often detected alongside with GABA, to give a better picture on excitatory-inhibitory balance of the CNS. These neurotransmitters can be detected with different high-performance liquid chromatography (HPLC) techniques, however, each of them has its own drawback. Electrochemical detection represents the least applied method, as the use of such detectors can be very circumstantial, however, an advantage could be the high sensitivity, which can be further increased by using appropriate derivatizing agents (Polta \& Johnson, 1983; Clarke et al., 1999). Previously, the ion exchange chromatography method seemed to take its place, as it provides simplicity in sample preparation and high reproducibility, but the long running time was its main drawback (Fekkes et al., 2000). Nevertheless, in the recent years these methods are used less, as some derivatization agents seem to provide the same sensitivity via the application of fluorescent light detector (FLD), a detection method that is simpler and more widely applied in a reverse-phase HPLC. One of the mainly used derivatization agents is the ophthalaldehyde (OPA), in the presence of 3-mercaptopropionic acid ((3MP; (de Freitas Silva et al., 2009; Perucho et al., 2015; Stragierowicz et al., 2017; Veres et al., 2019)), as it furnishes fast reactions, its derivates can be obtained in aqueous solutions at ambient temperature and they are fluorescent compounds with high selectivity and sensitivity (Molnár-Perl, 2011). Furthermore, it does not break down or react further to form byproducts if it is added in excess (Cooper et al., 1984). Although its disadvantage may be its unstable character and sensitive reaction to the change of $\mathrm{pH}$ in sample preparation (Molnár-Perl, 2011), OPA can be used pre- 
column, yielding a relatively short running time resulting in a relatively easy simultaneous measurement of Glu and GABA (Veres et al., 2019).

The determination of the concentrations of various TRP metabolites, including KYNA, from biological matrices represents a great challenge due to their distinct chemical properties or their different concentrations in samples (Sadok et al., 2017). The main problem of method development was the determination of metabolites in one single run which mostly needed a multi-step sample preparation and/or a complex instrumental background, such as the gas chromatography-mass spectrometry (Sano et al., 2014), ultra- or HPLC mass spectrometry (Tömösi et al., 2020). The latter one is a costly method, and in each case requires a longer sample preparation time. However, even fluorescent detection sometimes necessitates a pre-, on- or post-column derivatization, which gives fluorescent metabolites at the end of the procedure. Therefore, the detection with FLD can yield lower limit of detection (LOD) value (Mawatari et al., 1989; Mitsuhashi et al., 2006; Xiao et al., 2008a), which may have a special importance especially in light of low sample amount in several cases (e.g. mouse CNS samples). Accordingly, the partial assessment of the KP is a widely applied approach using simple HPLC methods with different detection techniques, including the UV detector (UVD), diode array detector, FLD (Zhao et al., 2011; Veres et al., 2015; Sadok et al., 2017), or electrochemical detector (Zhang et al., 2009). The quantification of some TRP metabolites with HPLC was first described by Werner (Werner et al., 1987), who measured TRP, KYN, 3-hydroxy anthranilic acid (3HANA) and anthranilic acid using FLD and UVD, by changing the wavelengths in time, and later by Hervé (Hervé et al., 1996), who included the use of two different detectors simultaneously, and described a method suitable for the detection of TRP, KYN, KYNA, 3HANA and 3-hydroxy kynurenine from standard solution. However, in a real-life situation this latter method was only suitable for the detection of TRP, KYN, KYNA and 3HANA from human serum. The first method which assessed some KP metabolites from tissue samples and used internal standard (IS) was described by Werner (Werner-Felmayer et al., 1989), who applied 3-nitro-L-tyrosine (3NLT) regarding UVD, as its structure is very similar to that of KYN (Figure 3). 
<smiles>Nc1ccccc1C(=O)C[C@H](N)C(=O)O</smiles>

KYN<smiles>O=C(O)c1cc(O)c2ccccc2n1</smiles>

KYNA<smiles>N[C@@H](Cc1ccc(O)c([N+](=O)[O-])c1)C(=O)O</smiles>

3NLT<smiles>O=C(O)c1nc(O)c2ccccc2n1</smiles>

HCA

Figure 3. Similarities between the UVD IS, 3NLT and KYN, and FLD IS, HCA and KYNA. 3NLT 3-nitro-L-tyorisne, HCA 4-hydroxyquinolizone carboxylic acid, KYN kynurenine, KYNA kynurenic acid.

Accordingly, 3NLT remained one of the most widely applied ISs for the HPLC methods suitable for KP metabolite detection, as its use does not interfere with any other metabolite. Beside this, some researchers used norvaline (Myint et al., 2007), methyl-tryptophan (Dazzi et al., 2001; Vignau et al., 2004; Dario et al., 2017) and creatine (Zhao et al., 2011; Zhao, 2013). In case of biological samples, the application of ISs is essential as these yield the only way to calculate the sample loss during sample preparation and analysis. Nonetheless, only $37.7 \%$ of the articles of interest applied ISs at all (Cseh et al., 2019), and none of them utilized ISs separately for each detector. This may be due to that two requirements of the ISs are rarely taken into account: compatibility with the detector response and similarity in structure and properties with the analyzed compounds, beside the obviously necessary features of ISs (stability, pure form, absence in native sample, or no interference with another compound) (Dolan, 2012). Accordingly, the application of different ISs is required for each detector as the concentrations are calculated from a calibration plot where the concentration values are plotted against the response ratios. In case of the above-mentioned partial assessment of TRP metabolites, TRP, 5-HT and KYNA are detected by FLD, whereas KYN by UVD, and accordingly, at least 2 ISs should be applied during their detection. In light of these requirements, 3NLT is appropriate for the UVD, whereas a newly synthetized compound, 4hydroxyquinazoline-2-carboxylic acid (HCA) was utilized for FLD (Cseh et al., 2019) (Figure 3).

Regarding all the above-mentioned analytical procedures, a detailed validation process, including at least selectivity, linearity, LOD, limit of quantification (LOQ), precision and 
recovery, is essential to be able to determine the robustness of the developed method in harmonization with the official guidelines (International Conference on Harmonization, 2005). 


\section{Aims}

The aims of our study were as follows:

(i) To explore the neurochemical profile of CFA-induced orofacial pain in rats, including the assessment of Glu, GABA, TRP, 5-HT, KYN and KYNA, and finding the shift point regarding small molecule neurotransmitter concentration changes versus that of the previously described pain-related neuropeptides.

(ii) To optimize and validate a HPLC-UVD/FLD method for the determination of TRP, 5-HT, and that of the neuroprotective branch of the KP from several different biological matrices, including mouse and rat CNS and plasma, and human CSF and plasma, by using two ISs, one for each detector. 


\section{Materials and methods}

\subsection{CFA model of orofacial pain}

Twenty-seven young adult (10-12 weeks old, 250-300 g) male Sprague-Dawley rats (Charles River Laboratories, Wilmington, MA, USA), were used for the experiments. The animals were bred and maintained under standard laboratory conditions with $12 \mathrm{~h}-12 \mathrm{~h}$ light/dark cycle at 24 $\pm 1^{\circ} \mathrm{C}$ and $50 \%$ relative humidity, 3 animals per each home cage in the Laboratory Animal House of the Department of Neurology, University of Szeged. The rats had free access to standard rat chow and water. The experiment was not pre-registered. All experimental procedures performed in this study complied fully with the guidelines of Act 1998/XXVIII of the Hungarian Parliament on Animal Experiments (243/1988) and with the recommendations of the International Association for the Study of Pain and European Communities Council (86/609/ECC). The studies were in harmony with the Ethical Codex of Animal Experiments and were approved by the Ethics Committee of the Faculty of Medicine, University of Szeged, with a permission number of XI./1102/2018. Complete Freund adjuvant (CFA; killed mycobacteria suspended in paraffin oil, $1 \mathrm{mg} / \mathrm{ml}$ ) was obtained from Sigma-Aldrich (St. Louis, MO, USA), and $50 \mu \mathrm{l}$ was administered per animal. We tried to minimize the use of animals by adopting the key aspects of the 3Rs (Replacement, Reduction and Refinement). Therefore, the experimental groups were added in a sequential manner, starting from $24 \mathrm{~h}$ following CFA administration with $24 \mathrm{~h}$ steps till the time point where the proposed alterations diminish. Therefore, no randomization was performed to allocate subjects in the study. By the end of the experiments we had three groups, one control and two with CFA treatment (Figure 4).

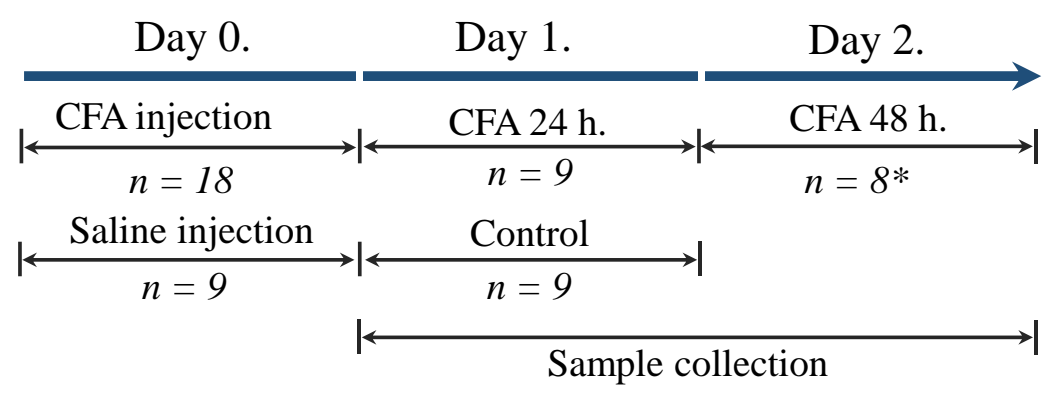

Figure 4. Time-line of the experimental procedure applied in this study. CFA Complete Freund's adjuvant, $n$ number of the animals per group. *One animal died in cage after CFA injection. 
The control (CO) group was chosen to be at $24 \mathrm{~h}$, as previous experiments demonstrated that there is no difference in the controls, when they do not receive PBS at all in the whisker pad vs. treated with PBS and perfused $24 \mathrm{~h}$ after treatment vs. treated with PBS and perfused $48 \mathrm{~h}$ after treatment ( $n=3$ animal/group; measured analytes: GABA and Glu, TRP metabolites).

The rats were anesthetized with intraperitoneal $4 \%$ chloral hydrate solution mainly based on its safe application (Sigma-Aldrich, St. Louis, MO, USA; $10 \mathrm{ml} / \mathrm{kg}$ body weight dose) in the morning and $50 \mu \mathrm{l}$ of CFA was injected into the right whisker pad. No other analgesic was applied, otherwise the activation/sensitization phenomena during pain processing, an essential characteristic of the CFA model as well, would have been influenced. Control rats were injected with an equal volume of saline. CSF was taken from the suboccipital cistern, including the control group $(n=9), 24(n=9)$ and 48 hours $(n=9$ initially, finally $n=8$ as one animal died during the experiment) applying the above-described anesthetic procedure after injection, and following that the animals were perfused transcardially with $200 \mathrm{ml}$ phosphate-buffered saline (PBS). The spinal tap procedures were unsuccessful in 5 occasions and 7 of the CSF samples were excluded from analysis due to contamination with blood. Accordingly, 5-5 samples remained in the control and CFA $24 \mathrm{~h}$ groups, and 4 in the CFA $48 \mathrm{~h}$ group for analysis. Therefore, this part of the study was only exploratory due to the low statistical power. Blood samples were taken from the left ventricle into ice-cold glass tubes containing disodium ethylenediaminetetraacetate dihydrate ( $\mathrm{Na}_{2}$ EDTA, Lach-Ner s.r.o, Neratovice, Chech Republic) and the plasma was separated by centrifugation ( 3500 RPM for $10 \mathrm{~min}$ at $4^{\circ} \mathrm{C}$ ). Following decapitation two different brain structures were dissected, the TNC and the somatosensory cortex ( $\mathrm{ssCX}$ ). Both right- and left-sided samples were separately removed on ice and stored at $-80^{\circ} \mathrm{C}$ until further use in each case. Prior to all measurements, during the tissue weighting or plasma/CSF precipitation process, all samples were relabeled, and a blind study was conducted, i.e., the experimenter was no aware of which samples were part of $\mathrm{CO}$ or $24 \mathrm{~h}$ groups. Therefore, in each case a randomization was applied as well. Validated HPLC measurements were performed during the experiment. First, the brain samples were homogenized in $0.5 \mathrm{M}$ perchloric acid (PCA), at 1:5 w/v containing the ISs (3NLT and HCA), applied in the measurement of TRP metabolites as detailed below. Then, supernatants were aliquoted and kept at $-80^{\circ} \mathrm{C}$ until the bioanalytical procedure. Regarding Glu and GABA measurements, $100 \mu 1$ of the brain supernatant was diluted to $1: 100 \mathrm{v} / \mathrm{v}$ with distilled water and $100 \mu 1$ of this dilution was derivatized with $100 \mu$ l solution ( 2 ml OPA (Sigma-Aldrich, Saint 
Louis, MO, USA), $7.94 \mathrm{ml} 0.2 \mathrm{M}$ borate puffer (pH = 9.9; Sigma-Aldrich, Saint Louis, MO, USA) and $60 \mu 1$ MP (Sigma-Aldrich, Saint Louis, MO, USA) and further diluted with $50 \mu 1$ distilled water containing the corresponding IS, the homoserine (Sigma-Aldrich, Saint Louis, MO, USA). For the separation, gradient elution was applied. Mobile phase 'A' was 95:5 v/v $0.05 \mathrm{M}$ sodium acetate $(\mathrm{pH}=5.5)$ :methanol, whereas mobile phase ' $\mathrm{B}$ ' was 45:45:10 v/v methanol:acetonitrile (ACN):water. ACN was purchased from Scharlau (Barcelona, Spain) and methanol from Sigma-Aldrich (Saint Louis, MO, USA). Chromatographic separations were performed on a Kinetex C18 150x4.6 i.d. $5 \mu \mathrm{m}$ particle size column (Phenomenex Inc., Torrance, CA, USA) after passage through a SecurityGuard pre-column C18, 4x3 mm i.d., 5 $\mu \mathrm{m}$ particle size (Phenomenex Inc., Torrance, CA, USA) applying gradient elution. The elution started with $95 \%$ ' $\mathrm{A}$ ' decreasing linearly to $50 \%$ then staying there for $2 \mathrm{~min}$ and reequilibrating to $95 \%$ in $1 \mathrm{~min}$ for a total $16 \mathrm{~min}$ runtime. The flow rate was $1 \mathrm{ml} / \mathrm{min}$, injection volume was $10 \mu \mathrm{l}$ and the FLD was set to $230 / 440 \mathrm{~nm}$ for excitation/emission wavelengths. The validation process was carried out as described previously (Veres et al., 2019). Regarding the Glu and GABA measurements from CFS samples, the initial amount of mobile phase 'A' applied for the brain samples was 95\%, but for CSF samples it was changed to 93\%, as coelution was observed under the initial circumstances. The ratios applied for the CSF sample preparation (1:1:0.5 = sample: derivatization solution: IS ) remained the same, similar to brain supernatants. For the TRP, 5-HT, KYN and KYNA measurements from brain samples, the mobile phase consisted of $200 \mathrm{mM}$ zinc-acetate (ZnAc) solution at $\mathrm{pH}$ 5.8, adjusted with acetic acid. The organic component (ACN) in the mobile phase was 5\%, and the solution was filtered through a cellulose membrane with $0.2 \mu \mathrm{m}$ pore size. The flow rate was $1.2 \mathrm{ml} / \mathrm{min}$ and the injection volume was $50 \mu 1$. During the measurement of CSF and plasma samples, TRP, KYN and KYNA was separated by a mobile phase similar to the one used for the CNS methods, except that $\mathrm{pH}$ was set at 6.2. The injection volume was $50 \mu \mathrm{l}$ and $20 \mu \mathrm{l}$, respectively.

\subsection{Quantification of TRP, 5-HT, KYN and KYNA from different biological samples}

Due to the importance of TRP and its metabolites in different neurological disorders and their animal model as well, paying a special attention to pain models, their analyses may be of interest, not only in rats, but in human and mouse samples too. Therefore, method optimization and validation processes are described below, regarding human plasma and CSF, rat plasma and CNS (brain and upper part of the cervical spinal cord), and mouse brain and plasma samples. 
All chromatographic analyses described below were performed using an Agilent 1100 HPLC system (Santa Clara, CA, USA) with Agilent G1314A UV detector (UVD) and G1321A fluorescent detector (FLD) attached. Chromatographic separations were performed on a Kinetex C18 150×4.6 i.d. $5 \mu \mathrm{m}$ particle size column (Phenomenex Inc., Torrance, CA, USA) after passage through a SecurityGuard pre-column $\mathrm{C} 18,4 \times 3 \mathrm{~mm}$ i.d., $5 \mu \mathrm{m}$ particle size (Phenomenex Inc., Torrance, CA, USA). The purity of all standards and solutions were analytical grade or HPLC grade. The IS used for the FLD, HCA, was synthesized at the Department of Pharmaceutical Chemistry, University of Szeged. Before the method optimization process, the spectral analyses of the UV-detected compounds were made with an Agilent 8453 UV-Vis Spectroscopy System (Santa Clara, CA, USA). The reference compounds, including the TRP, 5-HT, KYN, KYNA, 3NLT; PCA, ZnAc and phosphoric acid were purchased from Sigma-Aldrich (Saint Louis, MO, USA). ACN was obtained from Scharlau (Barcelona, Spain) and acetic acid from VWR International (Radnor, PA, USA). The di-sodium-hydrogen phosphate dihydrate was obtained from VWR International (Radnor, PA, USA) and potassium dihydrogen phosphate from Applichem Panreac (Darmstadt, Germany). Regarding the mobile phase, it consisted of $200 \mathrm{mM} \mathrm{ZnAc}$ solution at $\mathrm{pH}$ of 6.2 for human and murine plasma and CSF samples, and at $\mathrm{pH}$ of 5.8 for murine CNS samples, the $\mathrm{pH}$ value was adjusted with acetic acid. The organic component (ACN) in the mobile phase was 5\%, and the solution was filtered through a cellulose membrane with $0.2 \mu \mathrm{m}$ pore size. The flow rate was $1.2 \mathrm{ml} / \mathrm{min}$ and $20 \mu \mathrm{l}$ of the plasma supernatants were injected, whereas in case of CSF and CNS homogenate the injection volume was $50 \mu$ l. In each case, two ISs were applied: 3NLT for the UVD, and HCA for the FLD, both chosen by their similarities with one of the metabolites detected (Introduction; Figure 3).

We applied ZnAc at a relatively high concentration $(200 \mathrm{mM})$, which is in the middle of the 100-500 mM, a range widely used in previously described methods (Sadok et al., 2017), as $\mathrm{ZnAc}$ increases the fluorescence intensity of KYNA by creating a complex with the $\mathrm{Zn}^{2+}$ ions, which seems essential for its detection above LOQ (Figure 5). However, it is worth to mention that we focused on the prevention of precipitation as well and we did not observe any signs of precipitation and the lifespan of the applied column was not affected at all. 


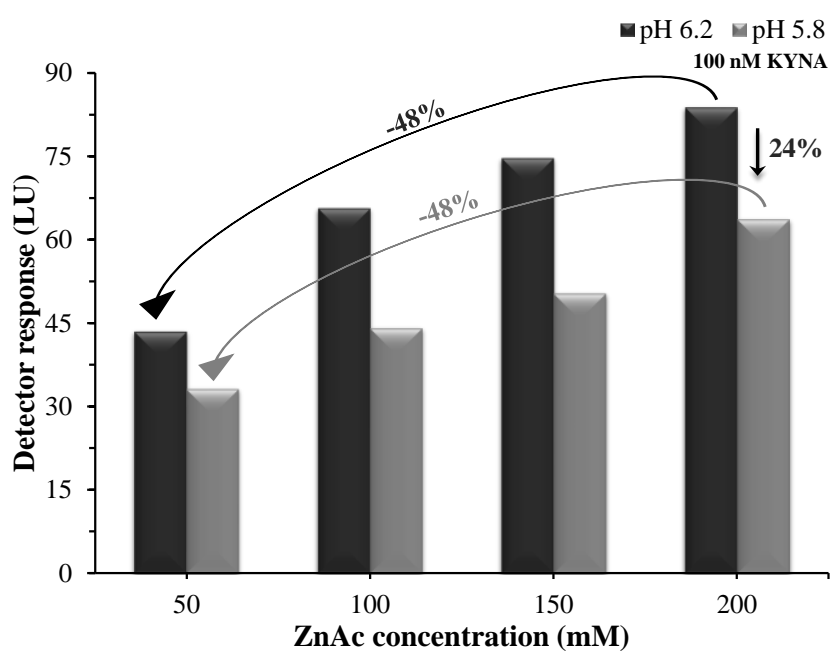

Figure 5. The effect of ZnAc on the achieved signal intensity for KYNA. KYNA kynurenic acid, $L U$ luminescence, $Z n A c$ zinc acetate.

Not only the $\mathrm{ZnAc}$, but the applied $\mathrm{pH}$ value seemed to have a large influence on the sensitivity of KYNA detection as well (Figure 6), as its reduction was necessary during the development of the method suitable for the brain samples.

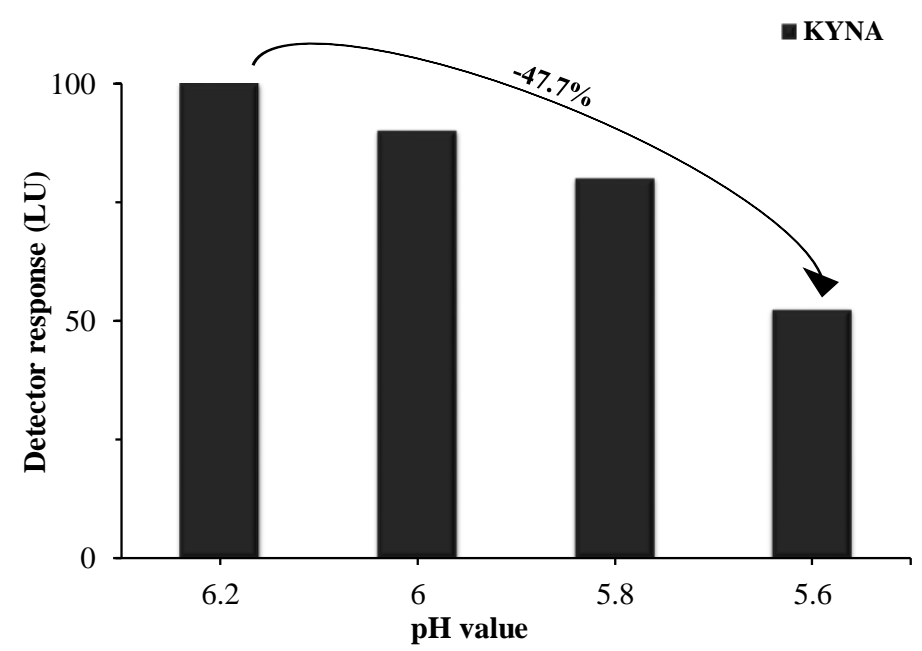

Figure 6. The effect of $\mathrm{pH}$ on the achieved signal intensity for KYNA. KYNA kynurenic acid, $L U$ luminescence, $Z n A c$ zinc acetate.

Furthermore, not only the $\mathrm{pH}$ value was changed during the method development, but we tested different water phase:organic phase ratios as well, therefore the effect of the ACN on the sensitivity of KYNA detection was also assessed (Figure 7). However, it did not have such a large impact as the $\mathrm{ZnAc}$ concentration and $\mathrm{pH}$ value on the sensitivity of the methods. 


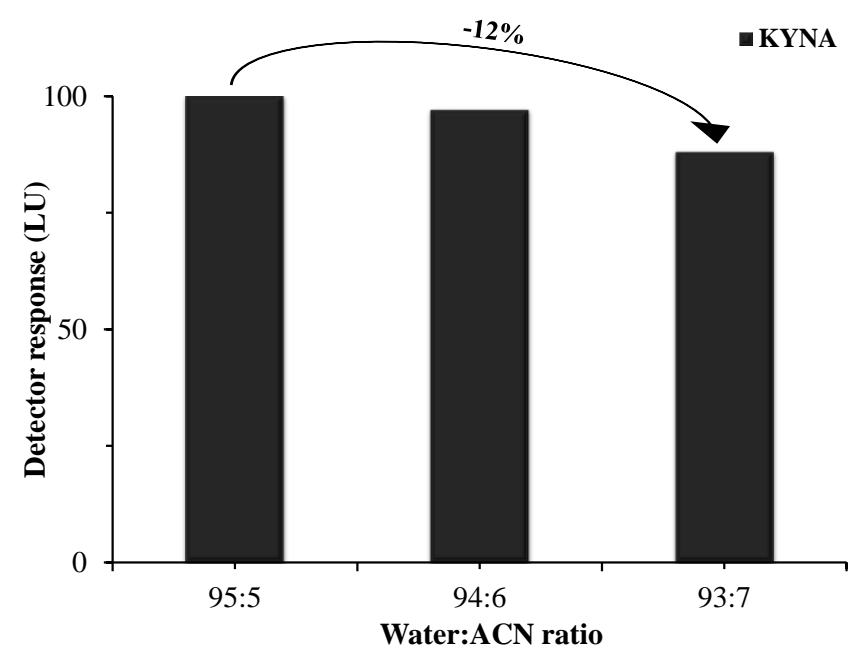

Figure 7. The effect of ACN on the achieved signal intensity for KYNA. ACN acetonitrile, KYNA kynurenic acid, $L U$ luminescence.

As the $\mathrm{pH}$ value was changed during the method optimization process, the UV-Vis spectra data were collected from $200-800 \mathrm{~nm}$ in cases of KYN and 3NLT to determine the optimal wavelengths for measurements (Figure 8A and B), whereas the determination of optimal wavelengths in case of FLD was carried out via the collection of spectral data in the ranges of 220-380 nm (excitation) and 300-495 nm (emission) for each fluorescent compound, i.e., TRP, 5-HT, KYNA and HCA (Figure 9). For the two different pH values, the metabolites showed different optimal detection wavelengths.
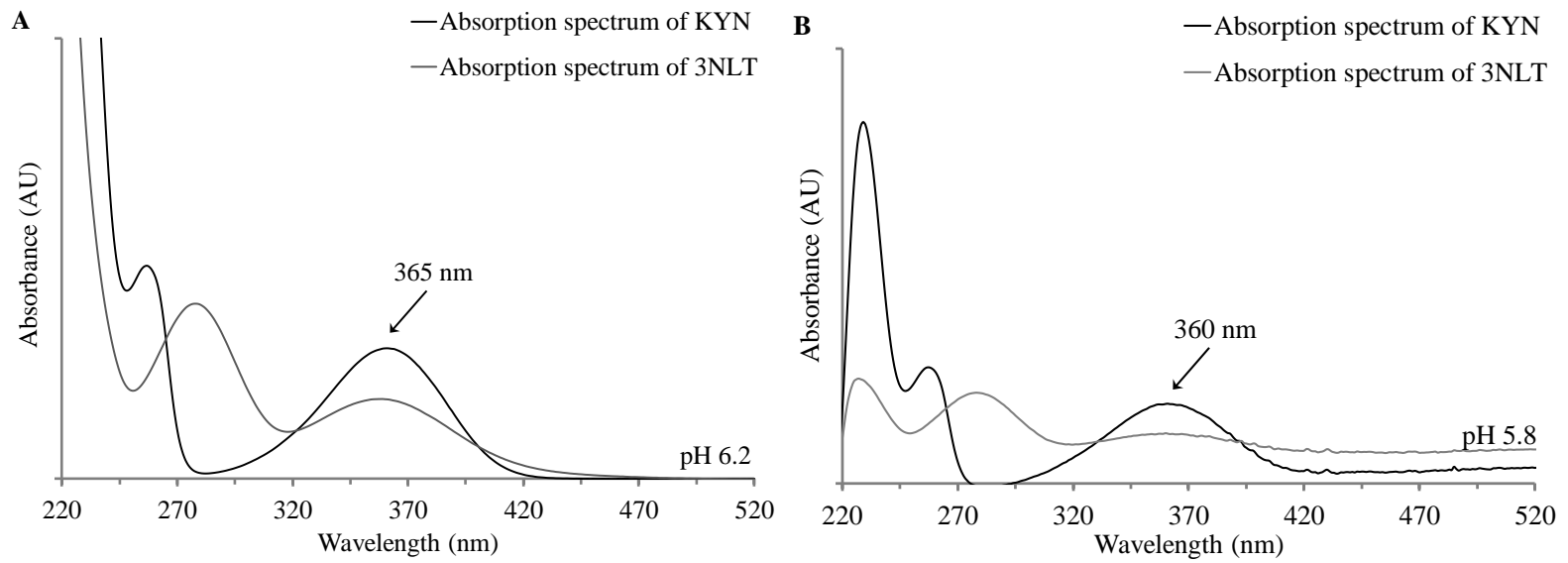

Figure 8. Absorption spectral analyses of KYN and 3NLT, with mobile phase pH set at 6.2 (A) and 5.8 (B). 3NLT 3-nitro- $L$-tyrosine, $A U$ absorbance unit, $K Y N$ kynurenine. 


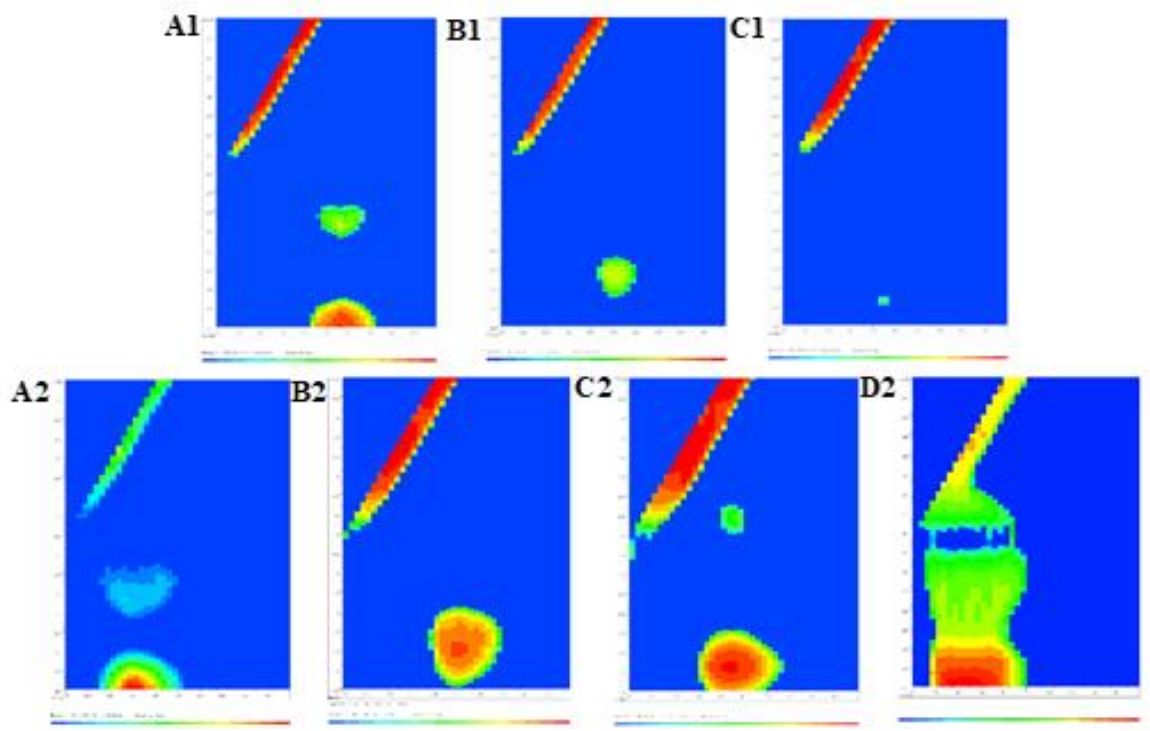

Figure 9. Fluorescence 3D spectral scan (for both mobile phases: $\mathrm{pH}$ 6.2: A1, B1, C1 and pH 5.8 A2, B2, C2, D2) of the quantified compounds (TRP, A; KYNA, B; HCA, C; 5-HT, D). X axis represents the emission from 300 to $480 \mathrm{~nm}$, whereas y axis the excitation from 220 to $380 \mathrm{~nm}$. Colors represent the intensity of luminescence from 300 (dark blue) to different intensity values (red). 5-HT serotonin,

HCA 4-hydroxyquinolizone carboxylic acid, KYNA kynurenic acid, TRP tryptophan

Following the assessment of all parameters, the most appropriate ones were chosen in case of the brain samples as well (Figure 10), and the validation process was completed.
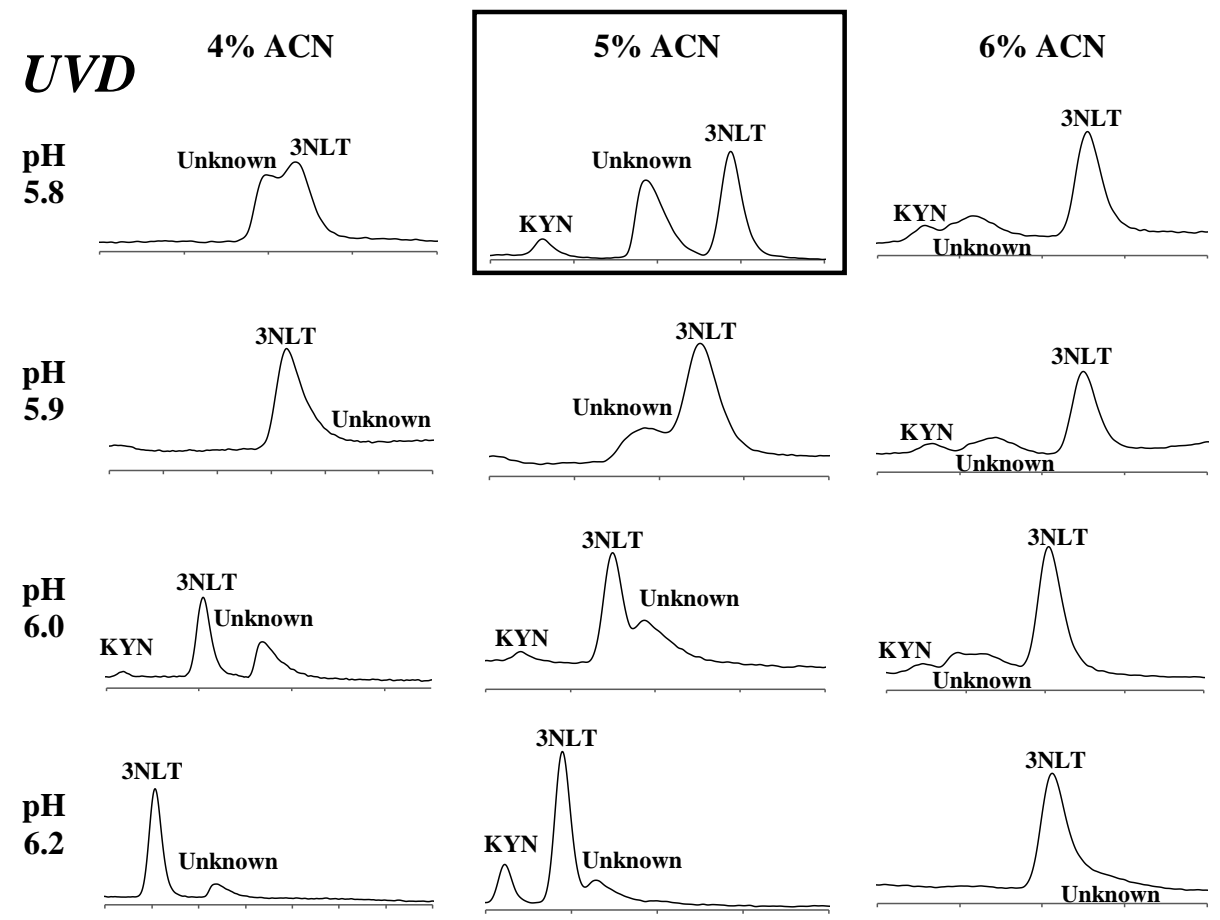

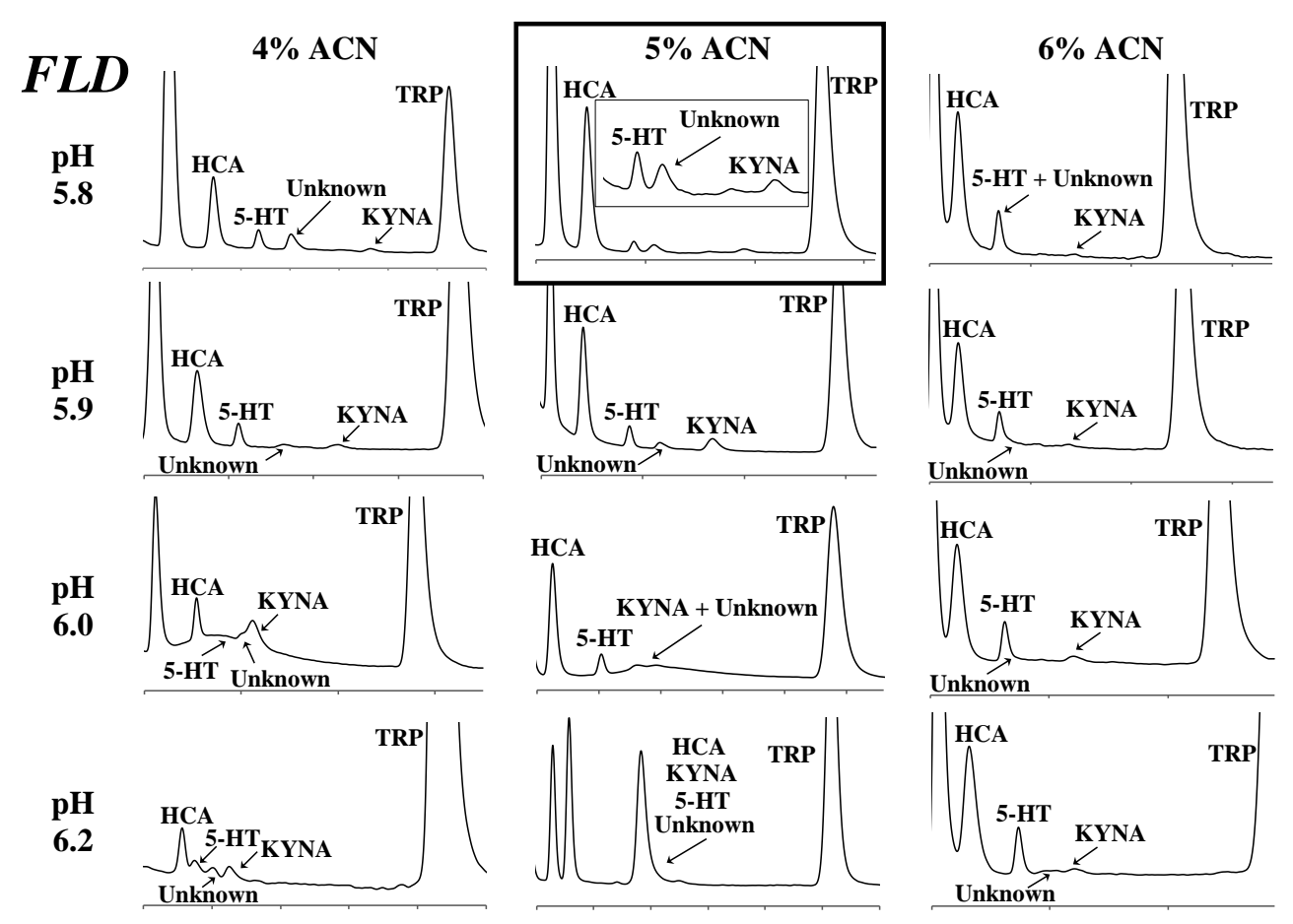

Figure 10. The UVD and FLD chromatograms of pooled rodent brain samples, in different conditions.

The $\mathrm{X}$ axis represents the running time of a sample, whereas the $\mathrm{Y}$ axis the detector response (mAU for the UVD, LU for the FLD). 3NLT 3-nitro-L-tyrosine, 5-HT serotonin, ACN acetonitrile, FLD fluorescence detector, $H C A$ 4-hydroxyquinazoline-2-carboxylic acid, KYN kynurenine, KYNA kynurenic acid, $L U$ luminescence, $m A U$ mili absorbance unit, TRP tryptophan, $U V D$ UV detector.

During the method development and validation process, the used solutions were made from stock solutions of $100 \mu \mathrm{M}$ prepared by dissolving accurately weighed standard compounds in 0.5 M PCA solution, except the KYNA, which was dissolved in phosphorous buffer, with $\mathrm{pH}$ set at 6.2 with $85 \%$ phosphoric acid, due to solubility issues. A series of working solutions (WS) of the analytes with different concentration ranges for each matrix was prepared containing the ISs at final concentration of $2 \mu \mathrm{M}$ for $3 \mathrm{NLT}$ and $100 \mathrm{nM}$ for HCA. During the validation process, for the calibration curve, which is further used for LOD and LOQ value determination, six calibration standards were prepared by spiking the respective WSs into blank biological matrices, i.e., blank human and rat plasma and CSF, and blank mouse plasma, respecting the same dilution ratios as the ones applied in the sample preparation as well. Due to hard sampling and contamination issues, the amount of the obtained rat CSF samples was enough only for the linearity study, along with the LOD and LOQ determination. With regard to rat and mouse CNS samples, the first step was the homogenization of the respective CNS regions, and thereafter the appropriate amount of the WSs was added to the supernatant. TRP, 
5-HT, KYN and KYNA were prepared in the presented final concentration ranges (Table 1). The peak area response ratios were plotted against the corresponding concentration, and the linear regression computations were carried out by the least square method with the freely available R software (R Development Core Team, 2002).

Table 1. The concertation range applied for different biological matrices

\begin{tabular}{|c|c|c|}
\hline Biological sample & Analyte & Concentration range \\
\hline \multirow{3}{*}{ Human plasma } & TRP $(\mu \mathrm{M})$ & $5-50$ \\
\hline & $\mathrm{KYN}(\mu \mathrm{M})$ & $0.1-5$ \\
\hline & KYNA (nM) & $2-100$ \\
\hline \multirow{3}{*}{ Human CSF } & TRP $(\mu \mathrm{M})$ & $0.1-5$ \\
\hline & $\mathrm{KYN}(\mu \mathrm{M})$ & $0.05-3$ \\
\hline & KYNA (nM) & $2-60$ \\
\hline \multirow{3}{*}{ Mouse plasma } & $\mathrm{TRP}(\mu \mathrm{M})$ & $1-30$ \\
\hline & $\mathrm{KYN}(\mu \mathrm{M})$ & $0.05-3$ \\
\hline & KYNA (nM) & $2-100$ \\
\hline \multirow{4}{*}{ Mouse brain } & TRP ( $\mu \mathrm{M} ; \mathrm{nmol} / \mathrm{g} w w)$ & $0.2-10 ; 0.816-40.8$ \\
\hline & 5-HT ( $\mu \mathrm{M} ; \mathrm{nmol} / \mathrm{g}$ ww $)$ & $0.25-1 ; 0.102-4.08$ \\
\hline & KYN ( $\mu \mathrm{M} ;$ nmol/g ww) & $0.1-3 ; 0.408-12.2$ \\
\hline & KYNA (nM; pmol/g ww) & $0.5-60 ; 2-245$ \\
\hline \multirow{3}{*}{ Rat plasma } & TRP $(\mu \mathrm{M})$ & $1-60$ \\
\hline & $\mathrm{KYN}(\mu \mathrm{M})$ & $0.1-5$ \\
\hline & KYNA (nM) & $1-100$ \\
\hline \multirow{4}{*}{ Rat CNS } & TRP $(\mu \mathrm{M} ; \mathrm{nmol} / \mathrm{g} w \mathrm{w})$ & $0.1-5 ; 0.470-26.8$ \\
\hline & 5-HT ( $\mu \mathrm{M} ; \mathrm{nmol} / \mathrm{g}$ ww) & $0.25-1 ; 0.157-6.11$ \\
\hline & KYN ( $\mu \mathrm{M} ;$ nmol/g ww) & $0.1-3 ; 0.686-14.49$ \\
\hline & KYNA (nM; pmol/g ww) & $1-60 ; 4.79-322.21$ \\
\hline \multirow{3}{*}{ Rat CSF } & $\mathrm{TRP}(\mu \mathrm{M})$ & $1-60$ \\
\hline & $\mathrm{KYN}(\mu \mathrm{M})$ & $0.1-3$ \\
\hline & KYNA (nM) & $1-60$ \\
\hline
\end{tabular}

5-HT serotonin, CNS central nervous system, CSF cerebrospinal fluid, KYN kynurenine, $K Y N A$ kynurenic acid, TRP tryptophan, $w w$ wet weight.

The same procedure was applied for the preparation of the quality controls (QCs), i.e., spiking the blank biological matrices with the appropriate solutions, containing the analytes in three different concentration levels (low (LOQ), medium and high) for performing the accuracy assays. Both calibration standards and QCs were prepared freshly, on the day of the measurements, whereas stock solutions and WSs were stored at $-80^{\circ} \mathrm{C}$.

During the sample acquisition, mouse plasma samples and mouse brain tissues were obtained from 3-4 months old C57B1/6 mice. Rat plasma, CSF and CNS samples were obtained from 
10-12 weeks old male Sprague-Dawley rats. The blood samples were collected into Na-EDTAcontaining tubes and centrifuged at 3500 RPM for 10 min and the resulting plasma samples were stored at $-80^{\circ} \mathrm{C}$ until analysis. In both cases, frozen plasma was thawed at room temperature, then deproteinized with $0.5 \mathrm{M}$ PCA solution $(1: 1 \mathrm{v} / \mathrm{v})$, containing both ISs at final concentration of $100 \mathrm{nM} \mathrm{HCA}$ and $2 \mu \mathrm{M} 3 \mathrm{NLT}$, and centrifuged for $10 \mathrm{~min}$ at $12000 \mathrm{RPM}$ at $4^{\circ} \mathrm{C}$. For the validation process, the individual samples were pooled, whereas for the demonstration of the applicability of the method and comparison of the obtained results with those from the literature, the metabolites of interest were measured from 8 independent samples. Regarding the freshly prepared mouse brain and rat CNS samples, the tissues were weighed and then sonicated for $90 \mathrm{~s}$ in an ice-cooled solution, 1:5 w/v, comprising 0.5 M PCA and the 2 ISs in an Eppendorf tube. The content of the Eppendorf tube was centrifuged for $10 \mathrm{~min}$ at $12000 \mathrm{RPM}$ at $4^{\circ} \mathrm{C}$. For the validation process, pooled CNS homogenates were applied, whereas the applicability of the method was tested on 8 independent CNS samples, obtained from the same mice and rats as used for plasma sample measurements. The animal experiments were authorized by the local ethical committee of University of Szeged with adherence to the NIH guidelines and the EU directive 2010/63/EU for the protection of animals used for scientific purposes.

Human plasma samples were obtained from 26-39 years old healthy subjects following obtaining written informed consent. Sample handling was almost the same as in case of mouse plasma samples, only the deproteinization process differed somewhat (the ratio of plasma and $0.5 \mathrm{M}$ PCA solution was 1:3 v/v). The assessment of the applicability of the method was also carried out on 8 independent samples. The CSF samples were taken from 17-71 years old patients with headache who were initially suspected to have subarachnoid hemorrhage and underwent a spinal tap, but the CSF analysis was negative. Written informed consent was also obtained in each case. For the CSF samples, the same preparation procedure was applied as in cases of plasma samples, except using a dilution of 5:6 v/v. The applicability of the method was also tested on 8 independent CSF samples. All the human samples were obtained with the approval of the local Ethical Committee of the University of Szeged (46/2014), adhering to the tenets of the most recent revision of the Declaration of Helsinki.

\subsection{Statistics}

All statistical calculations were performed with the use of the freely available R software 3.5.3

(R Development Core Team). During the method validation and concentration calculation 
steps, the peak area response ratios were plotted against the corresponding concentration, and the linear regression computations were carried out by the least square method.

In the CFA study, the distribution of our data population was not determined as the applied statistical tests do not need assumptions regarding the distribution of underlying data. Accordingly, first we performed the Levene test to assess the homogeneity of variances. As the variances were equal, we performed a general independence test for two sets of variables measured on arbitrary scales, where the reference distribution was approximative based on the Monte-Carlo method. Afterwards, we carried out permutation t-tests as post hoc analysis for pairwise comparison. Permutations were applied via the Monte-Carlo method (10 000 random permutations) and Type I errors from multiple comparisons were controlled with false discovery rate. No test for outliers was conducted. With the key aspects of $3 R$ s in mind [43] we tried to keep the sample size as low as we can based on experiences from previous experiments. For every statistically significant result, we calculated the corresponding effect size (Cohen's d in this case) and based on its value, we decided whether the increase of sample size is necessary or not. 


\section{Results}

\subsection{CFA model of orofacial pain}

First of all, a short study was conducted to demonstrate that there are no differences in the level of the metabolites of interest, in either TNC or ssCX, between the three $\mathrm{CO}$ groups, i.e., shaminjected rats processed $24 \mathrm{~h}$ and $48 \mathrm{~h}$ following the injection and the treatment naïve group (Table 2). Therefore, in the experimental set-up, only the sham-injected rats, processed at $24 \mathrm{~h}$ after the treatment were included as a $\mathrm{CO}$ group, similar to the previous experiment on PACAP and CGRP in the same model (Körtési et al., 2019).

Table 2. The concentration of analyzed metabolites in a pilot study conducted on three different control groups

\begin{tabular}{|c|c|c|c|}
\hline Metabolites/groups & $\begin{array}{c}\text { Control } \\
0 \\
\end{array}$ & $\begin{array}{c}\text { Control } \\
24 \mathrm{~h}\end{array}$ & $\begin{array}{c}\text { Control } \\
48 \mathrm{~h}\end{array}$ \\
\hline \multicolumn{4}{|c|}{ Trigeminal nucleus caudalis } \\
\hline Glutamate $(\mu \mathrm{g} / \mathrm{g} \mathrm{ww})$ & $\begin{array}{c}703 \\
(697-711)\end{array}$ & $\begin{array}{c}712 \\
(667-746)\end{array}$ & $\begin{array}{c}689 \\
(676-709)\end{array}$ \\
\hline GABA $(\mu \mathrm{g} / \mathrm{g} w w)$ & $\begin{array}{c}139 \\
(138-140)\end{array}$ & $\begin{array}{c}140 \\
(121-143)\end{array}$ & $\begin{array}{c}132 \\
(126-133)\end{array}$ \\
\hline Tryptophan (nmol/g ww) & $\begin{array}{c}18.8 \\
(18.5-18.8)\end{array}$ & $\begin{array}{c}19.1 \\
(18.6-19.8)\end{array}$ & $\begin{array}{c}19.9 \\
(19.6-20.5)\end{array}$ \\
\hline Serotonin (nmol/g ww) & $\begin{array}{c}3.74 \\
(3.71-3.86)\end{array}$ & $\begin{array}{c}3.69 \\
(3.32-4.06)\end{array}$ & $\begin{array}{c}3.64 \\
(3.63-3.66)\end{array}$ \\
\hline Kynurenine (nmol/g ww) & $\begin{array}{c}0.520 \\
(0.509-0.545)\end{array}$ & $\begin{array}{c}0.626 \\
(0.353-0.660)\end{array}$ & $\begin{array}{c}0.615 \\
(0.400-0.760)\end{array}$ \\
\hline Kynurenic acid (pmol/g ww) & $\begin{array}{c}20.4 \\
(12.5-26.0) \\
\end{array}$ & $\begin{array}{c}10.8 \\
(6.50-31.4) \\
\end{array}$ & $\begin{array}{c}14.1 \\
(6.59-41.3) \\
\end{array}$ \\
\hline \multicolumn{4}{|c|}{ Somatosensory cortex } \\
\hline Glutamate $(\mu \mathrm{g} / \mathrm{g} w w)$ & $\begin{array}{c}1166 \\
(1083-1175)\end{array}$ & $\begin{array}{c}1191 \\
(1163-1412)\end{array}$ & $\begin{array}{c}1228 \\
(1059-1328)\end{array}$ \\
\hline GABA $(\mu \mathrm{g} / \mathrm{g} w w)$ & $\begin{array}{c}181 \\
(159-196)\end{array}$ & $\begin{array}{c}215 \\
(188-214)\end{array}$ & $\begin{array}{c}179 \\
(117-181)\end{array}$ \\
\hline Tryptophan (nmol/g ww) & $\begin{array}{c}15.8 \\
(14.4-16.1)\end{array}$ & $\begin{array}{c}17.7 \\
(13.9-20.9)\end{array}$ & $\begin{array}{c}19.0 \\
(12.4-21.3)\end{array}$ \\
\hline Serotonin (nmol/g ww) & $\begin{array}{c}2.56 \\
(2.53-2.93)\end{array}$ & $\begin{array}{c}2.86 \\
(2.52-2.83)\end{array}$ & $\begin{array}{c}2.87 \\
(2.34-3.20)\end{array}$ \\
\hline Kynurenine (nmol/g ww) & $\begin{array}{c}0.556 \\
(0.389-0.563)\end{array}$ & $\begin{array}{c}0.504 \\
(0.461-0.837)\end{array}$ & $\begin{array}{c}0.459 \\
(0.375-0.636)\end{array}$ \\
\hline Kynurenic acid (pmol/g ww) & $\begin{array}{c}20.8 \\
(12.2-21.5)\end{array}$ & $\begin{array}{c}10.9 \\
(10.6-13.1)\end{array}$ & $\begin{array}{c}10.2 \\
(9.3-10.6)\end{array}$ \\
\hline
\end{tabular}

$O$ naïve group, $24 h$ sham-injected rats, processed at $24 \mathrm{~h}$ after treatment, $48 h$ sham-injected rats, processed at $48 \mathrm{~h}$ after treatment, $w w$ wet wight. 
Both contralateral and ipsilateral CNS regions were measured separately, but we did not find significant differences in concentrations of any of the metabolites between the two sides, so the coherent data were pooled for further analysis. Therefore, the concentration values presented in Table 3 demonstrate the mean values of the two analyzed sides of each CNS region.

Table 3. Concentration levels of the measured metabolites in the analyzed brain regions

\begin{tabular}{|c|c|c|c|}
\hline & $\begin{array}{c}\text { Control group } \\
(\mathrm{n}=9)\end{array}$ & $\begin{array}{c}\text { CFA 24h } \\
(\mathrm{n}=9)\end{array}$ & $\begin{array}{c}\text { CFA } 48 \mathrm{~h} \\
\left(\mathrm{n}=8^{\dagger}\right)\end{array}$ \\
\hline \multicolumn{4}{|c|}{ Trigeminal nucleus caudalis (TNC) } \\
\hline Glu ( $\mu \mathrm{g} / \mathrm{g}$ ww) & $\begin{array}{c}684 \\
(644-746)\end{array}$ & $\begin{array}{c}772^{*, \#} \\
(742-859)\end{array}$ & $\begin{array}{c}731 \\
(687-745)\end{array}$ \\
\hline GABA $(\mu \mathrm{g} / \mathrm{g} w w)$ & $\begin{array}{c}167 \\
(154-187)\end{array}$ & $\begin{array}{c}180 \\
(174-235)\end{array}$ & $\begin{array}{c}167 \\
(164-171)\end{array}$ \\
\hline TRP (nmol/g ww) & $\begin{array}{c}20.3 \\
(19.2-22.4)\end{array}$ & $\begin{array}{c}20.3 \\
(18.2-24.5)\end{array}$ & $\begin{array}{c}19.4 \\
(17.7-20.8)\end{array}$ \\
\hline KYN (nmol/g ww) & $\begin{array}{c}0.656 \\
(0.428-0.671)\end{array}$ & $\begin{array}{c}0.876^{*}, \# \\
(0.830-1.13)\end{array}$ & $\begin{array}{c}0.532 \\
(0.480-0.597)\end{array}$ \\
\hline KYNA (pmol/g ww) & $\begin{array}{c}22.8 \\
(21.2-24.2)\end{array}$ & $\begin{array}{c}52.6^{* *, \#} \\
(34.6-72.3)\end{array}$ & $\begin{array}{c}25.8 \\
(21.9-28.8)\end{array}$ \\
\hline 5-HT (nmol/g ww) & $\begin{array}{c}2.99 \\
(2.92-3.33) \\
\end{array}$ & $\begin{array}{c}2.84 \\
(2.63-3.46) \\
\end{array}$ & $\begin{array}{c}3.32 \\
(3.09-3.44) \\
\end{array}$ \\
\hline \multicolumn{4}{|c|}{ Somatosensory cortex (ssCX) } \\
\hline Glu ( $\mu \mathrm{g} / \mathrm{g} w w)$ & $\begin{array}{c}1178 \\
(1082-1290)\end{array}$ & $\begin{array}{c}1269 \\
(1206-1397)\end{array}$ & $\begin{array}{c}1152 \\
(1052-1287)\end{array}$ \\
\hline GABA $(\mu \mathrm{g} / \mathrm{g} w w)$ & $\begin{array}{c}215 \\
(207-218)\end{array}$ & $\begin{array}{c}230 \\
(217-251)\end{array}$ & $\begin{array}{c}199 \\
(178-211)\end{array}$ \\
\hline TRP (nmol/g ww) & $\begin{array}{c}20.6 \\
(17.8-23.5)\end{array}$ & $\begin{array}{c}22.6 \\
(21.5-23.7)\end{array}$ & $\begin{array}{c}21.6 \\
(20.9-22.7)\end{array}$ \\
\hline KYN (nmol/g ww) & $\begin{array}{c}0.824 \\
(0.743-0.970)\end{array}$ & $\begin{array}{c}0.974 \\
(0.714-1.15)\end{array}$ & $\begin{array}{c}0.616 \\
(0.552-0.663)\end{array}$ \\
\hline KYNA (pmol/g ww) & $\begin{array}{c}16.2 \\
(9.70-18.8)\end{array}$ & $\begin{array}{c}27.3^{*, \#} \\
(17.3-39.3)\end{array}$ & $\begin{array}{c}9.73 \\
(7.01-12.8)\end{array}$ \\
\hline 5-HT (nmol/g ww) & $\begin{array}{c}2.55 \\
(1.66-2.68)\end{array}$ & $\begin{array}{c}2.27^{\#} \\
(2.17-2.53)\end{array}$ & $\begin{array}{c}2.89 \\
(2.65-3.17)\end{array}$ \\
\hline
\end{tabular}

Results are shown as median $\left(1^{\text {st }}-3^{\text {rd }}\right.$ quartile). ${ }^{\dagger}$ One animal died in cage after CFA injection. $* p<0.05$ vs. CO, ** $p<0.01$ vs. CO, \# $p<0.05$ vs. 48 h. 5-HT serotonin, CFA Complete Freund's adjuvant, GABA gamma-aminobutyric acid, Glu glutamate, KYN kynurenine, KYNA kynurenic acid, $n$ number of the animals per group, TRP tryptophan, $w w$ wet weight.

Regarding TNC, pairwise permutation t-tests following the independence tests revealed a significant elevation in the concentration of Glu $(p=0.0319$, Cohen's $d=1.49)$, KYN $(p=$ 0.0123, Cohen's $d=1.58)$ and KYNA $(p=0.0098$, Cohen's $d=1.92) 24 \mathrm{~h}$ following CFA 
injection compared to the controls and a significant decrease could be observed in Glu ( $\mathrm{p}=$ 0.0357, Cohen's $d=1.29)$, KYN ( $p=0.0123$, Cohen's $d=1.85)$ and KYNA $(p=0.0263$, Cohen's $\mathrm{d}=1.39$ ) levels by $48 \mathrm{~h}$ compared to the $24 \mathrm{~h}$ group, whereas there was no difference between the control and $48 \mathrm{~h}$ groups (Table 3, Figure 11).

\section{Trigeminal nucleus caudalis}

(TNC)
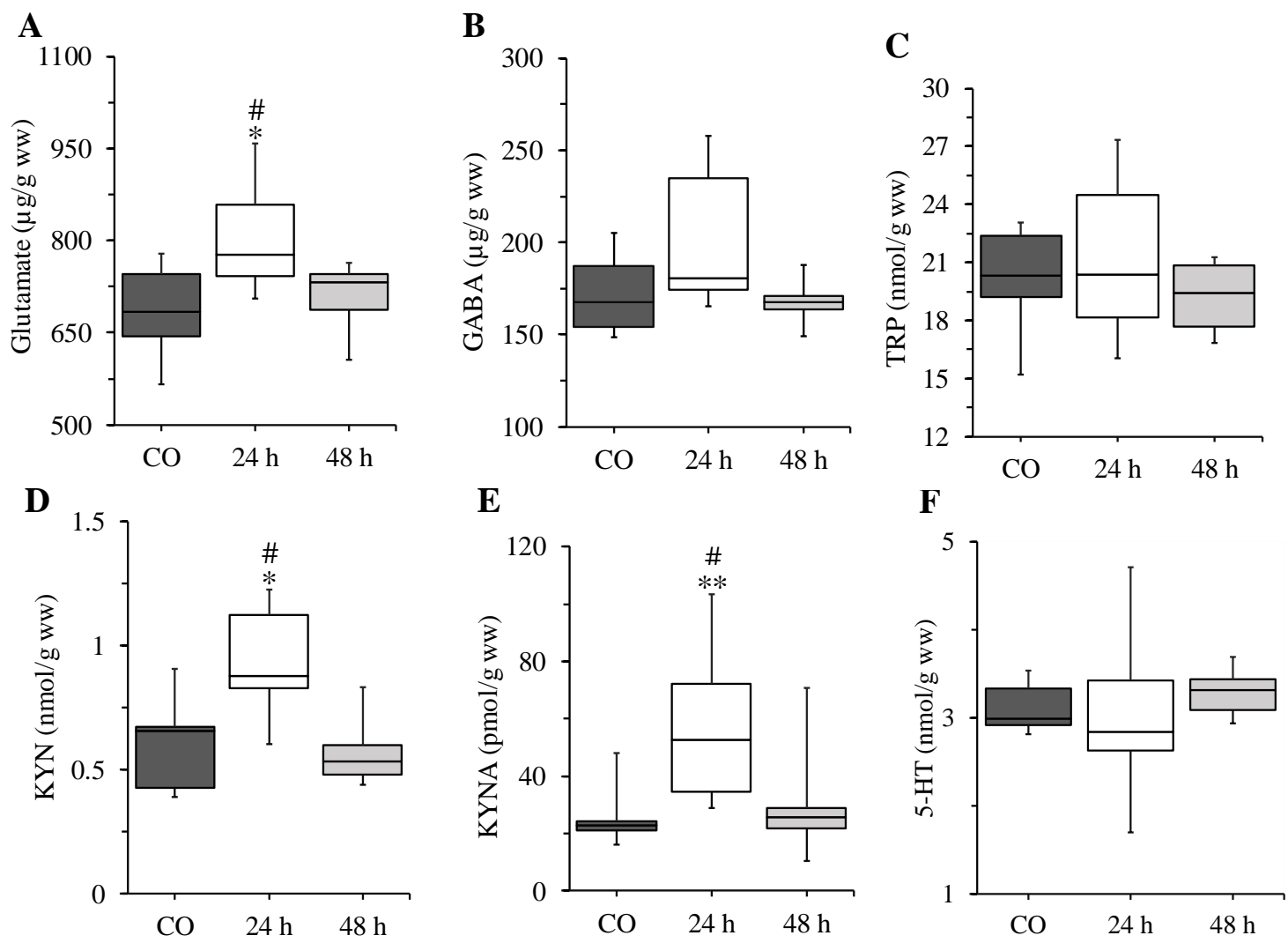

Figure 11. Concentration changes in the assessed metabolites in the TNC. ${ }^{*} p<0.05 v s$. CO, ${ }^{* *} p<$ 0.01 vs. CO, \# $p<0.05$ vs. $48 \mathrm{~h} . \mathrm{n}=9$ in the control and $24 \mathrm{~h}$ groups and $\mathrm{n}=8$ in the $48 \mathrm{~h}$ group. The boxplots are displayed as the intervals between the $1^{\text {st }}$ and $3^{\text {rd }}$ quartiles presenting the median values as well. 24 and $48 h$ CFA treated groups, 5-HT serotonin, GABA $\gamma$-aminobutyric acid, KYN kynurenine, KYNA kynurenic acid, $n$ number of the animals per group, TRP tryptophan, TNC trigeminal nucleus caudalis, $w w$ wet weight.

Regarding ssCX samples, an elevation in KYNA concentration $(p=0.0237$, Cohen's $d=1.36)$ could be observed $24 \mathrm{~h}$ following CFA administration, followed by a significant decrease by $48 \mathrm{~h}(\mathrm{p}=0.0173$, Cohen's $\mathrm{d}=1.80)$ and there was no difference between control and $48 \mathrm{~h}$ groups. Furthermore, in the ssCX, there was a significant increase in 5-HT levels in the $48 \mathrm{~h}$ 
group compared to the controls $(\mathrm{p}=0.0479$, Cohen's $\mathrm{d}=1.21)$ and to the $24 \mathrm{~h}$ group $(\mathrm{p}=$ 0.0479, Cohen's d = 1.20; Table 3, Figure 12). We calculated the KYN/TRP and KYNA/KYN ratios as well. The KYN/TRP ratio was significantly elevated in the $24 \mathrm{~h}$ group compared to the controls $(p=0.0419$, Cohen's $d=1.19)$ or to the 48 h group $(p=0.0419$, Cohen's $d=1.35$; Table 3). With regard to the KYNA/KYN ratio, there was no difference in any of the investigated biological matrices (data no shown).

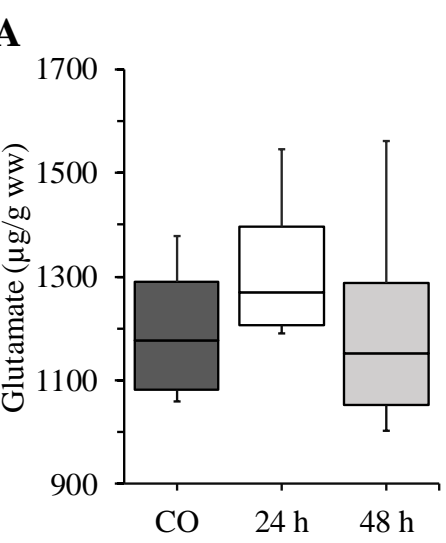

\section{Somatosensory cortex (ssCX)}
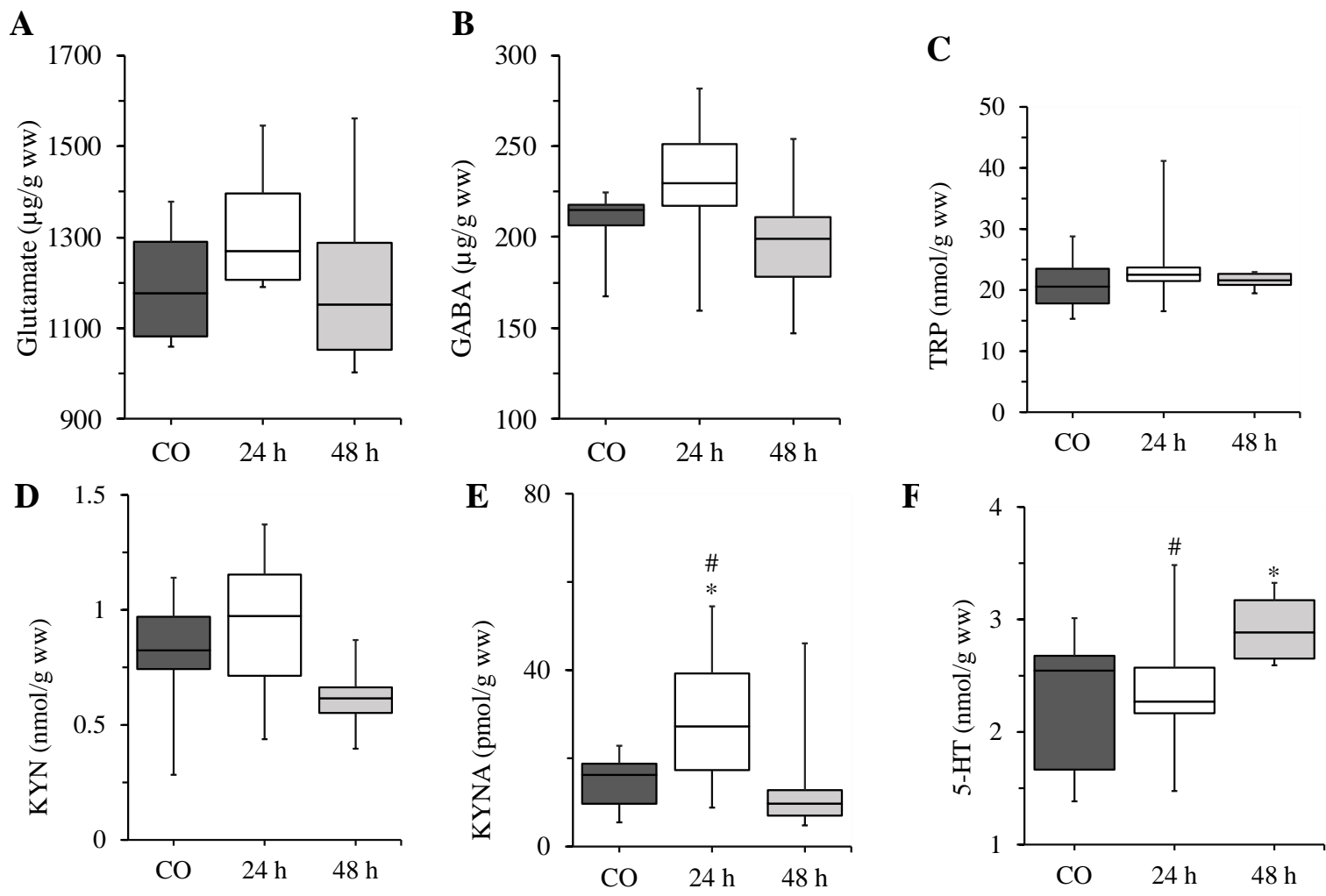

Figure 12. Concentration changes in the assessed metabolites in the somatosensory cortex. ${ }^{*} p<0.05$ vs. CO, \# $p<0.05$ vs. 48 h. $\mathrm{n}=9$ in the control and $24 \mathrm{~h}$ groups and $\mathrm{n}=8$ in the $48 \mathrm{~h}$ group. The boxplots are displayed as the intervals between the $1^{\text {st }}$ and $3^{\text {rd }}$ quartiles presenting the median values as well. 24 and $48 h$ CFA treated groups, 5- $H T$ serotonin, GABA $\gamma$-aminobutyric acid, KYN kynurenine,

KYNA kynurenic acid, $n$ number of the animals per group, TRP tryptophan, $w w$ wet weight.

Regarding CSF samples, TRP metabolites, Glu and GABA were measured. We found no significant alterations in the CSF, however, the power of the statistical tests in this case is low due to low case number ( $\mathrm{n}=5,5,4$ for control, $24 \mathrm{~h}$ and $48 \mathrm{~h}$ groups, respectively) and the concentration values of $\mathrm{KYN}$ in the control and CFA treated $48 \mathrm{~h}$ groups were below LOD 
$(0.107 \mu \mathrm{M})$, except one case from each group (Table 4; due to the low amount of 5-HT in the CSF samples, we could not quantify it, as the values were below LOD, LOD $=0.0274 \mu \mathrm{M}$ ).

Table 4. Concentration levels of the measured metabolites in the cerebrospinal fluid

\begin{tabular}{cccc}
\hline & $\begin{array}{c}\text { Control group } \\
(\mathrm{n}=5)\end{array}$ & $\begin{array}{c}\text { CFA 24 } \mathrm{h} \\
(\mathrm{n}=5)\end{array}$ & $\begin{array}{c}\text { CFA 48 h } \\
(\mathrm{n}=4)\end{array}$ \\
\hline & \multicolumn{2}{c}{ Cerebrospinal fluid } & \\
\hline \multirow{2}{*}{ Glu $(\mu \mathrm{M})$} & 6.08 & 9.87 & 8.61 \\
& $6.04-9.60$ & $4.90-16.5$ & $6.13-9.96$ \\
GABA $(\mu \mathrm{M})$ & 1.38 & 1.35 & 1.54 \\
& $1.04-1.49$ & $1.23-2.10$ & $1.40-1.73$ \\
TRP $(\mu \mathrm{M})$ & 1.40 & 1.32 & 1.55 \\
& $0.96-1.60$ & $1.24-3.77$ & $1.13-1.95$ \\
KYN $(\mu \mathrm{M})$ & $<\mathrm{LOD}$ & 0.21 & $<$ LOD \\
& 3.57 & $0.13-0.22$ & 4.10 \\
KYNA $(\mathrm{nM})$ & $1.61-11.0$ & 3.29 & $3.14-6.32$ \\
\hline
\end{tabular}

Results are shown as median ( $1^{\text {st }}-3^{\text {rd }}$ quartile). CFA Complete Freund's adjuvant, GABA gammaaminobutyric acid, $G l u$ glutamate, $K Y N$ kynurenine, $K Y N A$ kynurenic acid, $n$ number of animals per group, TRP tryptophan

In case of plasma samples, only the TRP metabolites were measured, and no significant differences were observed (Table 5).

Table 5. Concentration levels of the measured metabolites in the plasma samples

\begin{tabular}{cccc}
\hline & $\begin{array}{c}\text { Control group } \\
(\mathrm{n}=9)\end{array}$ & $\begin{array}{c}\text { CFA 24 h } \\
(\mathrm{n}=9)\end{array}$ & $\begin{array}{c}\text { CFA 48 h } \\
\left(\mathrm{n}=8^{\dagger}\right)\end{array}$ \\
\hline \multirow{2}{*}{ TRP $(\mu \mathrm{M})$} & Plasma & & 56.4 \\
& 63.9 & 81.4 & $(51.6-76.1)$ \\
KYN $(\mu \mathrm{M})$ & $(52.4-78.2)$ & $(54.3-88.1)$ & 3.27 \\
& 4.58 & 4.72 & $(2.83-4.79)$ \\
KYNA $(\mathrm{nM})$ & $(3.29-4.98)$ & $(4.45-5.12)$ & 139 \\
& 129 & 172 & $(95.0-173)$ \\
\hline
\end{tabular}

Results are shown as median ( $1^{\text {st }}-3^{\text {rd }}$ quartile). ${ }^{\dagger}$ One animal died in cage after CFA injection. $C F A$ Complete Freund's adjuvant, $K Y N$ kynurenine, KYNA kynurenic acid, $n$ number of animals per group, TRP tryptophan 


\subsection{Quantification of TRP, 5-HT, KYN and KYNA from different biological samples}

Due to the importance of the TRP metabolites, as presented in the previous parts, the validation process was carried out on six different biological matrices, including human plasma and CSF, mouse brain and plasma and rat CNS and plasma. During sample acquirement for the method optimization of rat samples, including the brain, CSF and plasma samples, a lot of unsuccessful CSF tap procedures were noted, therefore we did not collect enough CSF volume for the whole validation procedure. For the validation process, in addition to the linear equation calculated according to the applied concentration range (Table 1), LOD, LOQ (Table 6), intra- and interday precision were given as well (Table 7), along with the recovery values (Table 8). As a last step, the applicability of all methods was checked by comparing our data with literature data (Table 9).

LOD and LOQ were calculated by the equations $\mathrm{LOD}=3.3 \cdot \sigma / \mathrm{S}^{\prime}$ and $\mathrm{LOQ}=10 \cdot \sigma / \mathrm{S}^{\prime}$; where $\sigma$ is the standard error of the intercept and $S^{\prime}$ is the slope of the calibration curve of the analyte, presented in Table 6. Furthermore, a linearity study was conducted for rat CSF samples to determine LOD and LOQ values. Accordingly, the LOD and LOQ values for rat CSF were 31.1 and $102 \mathrm{nM}$ for TRP, 0.107 and $0.702 \mu \mathrm{M}$ for $\mathrm{KYN}$ and 1.04 and $3.45 \mathrm{nM}$ for KYNA, respectively, whereas 5-HT was undetectable in each case. 
Table 6. Limit of detection and limit of quantification values for tryptophan, serotonin, kynurenine and kynurenic acid in different biological matrices.

\begin{tabular}{|c|c|c|c|}
\hline Biological sample & Analyte & LOD & LOQ \\
\hline \multirow{3}{*}{ Human plasma } & TRP $(\mu \mathrm{M})$ & 1.04 & 3.14 \\
\hline & $\mathrm{KYN}(\mu \mathrm{M})$ & 0.100 & 0.303 \\
\hline & KYNA (nM) & 1.32 & 4.02 \\
\hline \multirow{3}{*}{ Human CSF } & $\mathrm{TRP}(\mu \mathrm{M})$ & 0.102 & 0.308 \\
\hline & $\mathrm{KYN}(\mu \mathrm{M})$ & 0.0274 & 0.0832 \\
\hline & KYNA (nM) & 1.23 & 3.72 \\
\hline \multirow{3}{*}{ Mouse plasma } & TRP $(\mu \mathrm{M})$ & 0.557 & 1.69 \\
\hline & $\mathrm{KYN}(\mu \mathrm{M})$ & 0.025 & 0.076 \\
\hline & KYNA (nM) & 1.33 & 4.03 \\
\hline \multirow{4}{*}{ Mouse brain } & TRP $(\mu \mathrm{M} ; \mathrm{nmol} / \mathrm{g}$ ww) & $0.204 ; 0.890$ & $0.619 ; 2.47$ \\
\hline & 5-HT ( $\mu \mathrm{M} ; \mathrm{nmol} / \mathrm{g}$ ww $)$ & $0.0086 ; 0.034$ & $0.102 ; 0.104$ \\
\hline & KYN ( $\mu \mathrm{M} ; \mathrm{nmol} / \mathrm{g} w w)$ & $0.0647 ; 0.259$ & $0.196 ; 0.785$ \\
\hline & KYNA (nM; pmol/g ww) & $0.456 ; 1.82$ & $1.38 ; 5.50$ \\
\hline \multirow{3}{*}{ Rat plasma } & $\operatorname{TRP}(\mu \mathrm{M})$ & 0.102 & 0.308 \\
\hline & $\mathrm{KYN}(\mu \mathrm{M})$ & 0.027 & 0.083 \\
\hline & KYNA (nM) & 1.23 & 3.72 \\
\hline \multirow{4}{*}{ Rat CNS } & TRP ( $\mu \mathrm{M} ; \mathrm{nmol} / \mathrm{g}$ ww) & $0.08 ; 0.36$ & $0.27 ; 1.21$ \\
\hline & 5-HT ( $\mu \mathrm{M} ; \mathrm{nmol} / \mathrm{g}$ ww $)$ & $0.031 ; 0.138$ & $0.102 ; 0.461$ \\
\hline & $\mathrm{KYN}(\mu \mathrm{M} ; \mathrm{nmol} / \mathrm{g} \mathrm{ww})$ & $0.04 ; 0.16$ & $0.12 ; 0.53$ \\
\hline & KYNA (nM; pmol/g ww) & $1.02 ; 4.62$ & $3.40 ; 15.4$ \\
\hline
\end{tabular}

5-HT serotonin, CSF cerebrospinal fluid, KYN kynurenine, KYNA kynurenic acid, LOD limit of detection, $L O Q$ limit of quantification, TRP tryptophan, $w w$ wet wight.

The precision of the method was determined for each analyte in all matrices (Table 6). Intraassay precision, expressed as $\mathrm{CV} \%$, was evaluated by running six consecutive replicates, whereas inter-assay precision was calculated by measuring the same samples used for the intraassay precision with separate calibrations curves, after three days.

Table 7. Intra-assay (CV\%) and inter-assay (bias\%) coefficients for tryptophan, serotonin, kynurenine and kynurenic acid in the different biological matrices.

\begin{tabular}{|c|c|c|c|c|c|c|c|c|}
\hline \multirow{2}{*}{ Sample type } & \multicolumn{2}{|c|}{ Tryptophan } & \multicolumn{2}{|c|}{ Serotonin } & \multicolumn{2}{|c|}{ Kynurenine } & \multicolumn{2}{|c|}{ Kynurenic acid } \\
\hline & $\mathrm{CV} \%$ & Bias\% & $\mathrm{CV} \%$ & Bias\% & $\mathrm{CV} \%$ & Bias\% & $\mathrm{CV} \%$ & Bias\% \\
\hline Human plasma & 1.14 & 3.23 & - & - & 2.81 & 6.37 & 2.01 & 2.05 \\
\hline Human CSF & 1.79 & 1.72 & - & - & 1.66 & 3.48 & 2.58 & 4.37 \\
\hline Mouse plasma & 1.36 & 1.19 & - & - & 2.59 & 1.33 & 3.24 & 4.27 \\
\hline Mouse brain & 2.41 & 1.11 & 4.25 & 52.4 & 2.32 & 6.16 & 3.42 & 3.79 \\
\hline Rat plasma & 1.27 & 1.98 & - & - & 3.98 & 4.59 & 2.08 & 2.04 \\
\hline Rat CNS & 1.03 & 5.99 & 1.91 & 19.73 & 4.00 & 10.6 & 3.70 & 5.96 \\
\hline
\end{tabular}

$C S F$ cerebrospinal fluid, $C V$ coefficient of variation 
Recovery studies were performed using spiked samples at three different concentration levels (LOQ, medium and high), with three replicates for each concentration. Recovery percentages were calculated as $\mathrm{R}=100 \times$ [(Css-Cns)/Cspike], where Css is the concentration in the spiked homogenate sample, whereas $\mathrm{Cns}$ is the concentration of the homogenate native sample (without spiking) and Cspike is the added concentration (Table 8).

Table 8. Recovery values (\%) of compounds in different biological matrices

\begin{tabular}{|c|c|c|c|c|c|}
\hline Conc. level of QCs & Analyte & Recovery (\%) & Conc. level of QCs & Analyte & $\begin{array}{c}\text { Recovery } \\
(\%)\end{array}$ \\
\hline \multicolumn{3}{|c|}{ Human plasma } & \multicolumn{3}{|c|}{ Human CSF } \\
\hline Low (LOQ) & \multirow{3}{*}{ TRP } & 116 & Low (LOQ) & \multirow{3}{*}{ TRP } & 116 \\
\hline Medium & & 109 & Medium & & 96.5 \\
\hline High & & 114 & High & & 106 \\
\hline Low (LOQ) & \multirow{3}{*}{$\mathrm{KYN}$} & 84.9 & Low (LOQ) & \multirow{3}{*}{ KYN } & 90.3 \\
\hline Medium & & 95.1 & Medium & & 86.7 \\
\hline High & & 99.2 & High & & 112 \\
\hline Low (LOQ) & \multirow{3}{*}{ KYNA } & 107 & Low (LOQ) & \multirow{3}{*}{ KYNA } & 90.2 \\
\hline Medium & & 94.3 & Medium & & 86.7 \\
\hline High & & 87.1 & High & & 111.7 \\
\hline \multicolumn{3}{|c|}{ Mouse plasma } & \multicolumn{3}{|c|}{ Rat plasma } \\
\hline Low (LOQ) & \multirow{3}{*}{ TRP } & 113 & Low (LOQ) & \multirow{3}{*}{ TRP } & 101 \\
\hline Medium & & 103 & Medium & & 103 \\
\hline High & & 107 & High & & 92.4 \\
\hline Low (LOQ) & \multirow{3}{*}{ KYN } & 82.5 & Low (LOQ) & \multirow{3}{*}{ KYN } & 104 \\
\hline Medium & & 110 & Medium & & 88.2 \\
\hline High & & 100 & High & & 107 \\
\hline Low (LOQ) & \multirow{3}{*}{ KYNA } & 104 & Low (LOQ) & \multirow{3}{*}{ KYNA } & 97.3 \\
\hline Medium & & 101 & Medium & & 101 \\
\hline High & & 115 & High & & 103 \\
\hline \multicolumn{3}{|c|}{ Mouse brain } & \multicolumn{3}{|c|}{ Rat brain } \\
\hline Low (LOQ) & \multirow{3}{*}{ TRP } & 107 & Low (LOQ) & \multirow{3}{*}{ TRP } & 106 \\
\hline Medium & & 103 & Medium & & 79.6 \\
\hline High & & 108 & High & & 108 \\
\hline Low (LOQ) & \multirow{4}{*}{ 5-HT } & 111 & Low (LOQ) & \multirow{4}{*}{ 5-HT } & 115 \\
\hline Medium & & 105 & Medium & & 90.3 \\
\hline High & & 103 & High & & 86.6 \\
\hline Low (LOQ) & & 113 & Low (LOQ) & & 86.2 \\
\hline Medium & \multirow{2}{*}{ KYN } & 111 & Medium & \multirow{2}{*}{ KYN } & 111 \\
\hline High & & 95.9 & High & & 115 \\
\hline Low (LOQ) & \multirow{3}{*}{ KYNA } & 97.8 & Low (LOQ) & \multirow{3}{*}{ KYNA } & 88.3 \\
\hline Medium & & 91.2 & Medium & & 109 \\
\hline High & & 93.8 & High & & 92.1 \\
\hline
\end{tabular}

Conc. concentration, $C S F$ cerebrospinal fluid, $K Y N$ kynurenine, $K Y N A$ kynurenic acid, $L O Q$ limit of quantification, $T R P$ tryptophan, $Q C$ quality control solution 
Regarding the applicability of the method, 8 independent samples were used. In case of rat CNS samples, we determined the concentrations by mixing different CNS regions, as in some cases, the literature data did not specify the brain regions used for the measurements (Table 9).

Table 9. The concentration of tryptophan, serotonin, kynurenine and kynurenic acid in different biological matrices, compared to literature data*

\begin{tabular}{|c|c|c|c|c|c|}
\hline \multicolumn{2}{|c|}{ Biological sample } & TRP & $5-\mathrm{HT}$ & KYN & KYNA \\
\hline $\begin{array}{l}\text { Human } \\
\text { plasma }^{\text {a }}\end{array}$ & $\begin{array}{l}\text { Current data } \\
\text { Literature data }\end{array}$ & $\begin{array}{c}43.2 \\
(36.7-49.7) \\
34.8-71.8\end{array}$ & - & $\begin{array}{c}2.14 \\
(1.90-2.35) \\
1.17-2.55\end{array}$ & $\begin{array}{c}26.8 \\
(23.9-32.9) \\
13.8-140\end{array}$ \\
\hline $\begin{array}{c}\text { Human } \\
\text { CSF }^{\mathrm{a}}\end{array}$ & $\begin{array}{l}\text { Current data } \\
\text { Literature data }\end{array}$ & $\begin{array}{c}2.72 \\
(2.04-3.31) \\
0.16-2.52\end{array}$ & - & $\begin{array}{c}0.0836 \\
(0.0586-0.109) \\
0.03-1.15\end{array}$ & $\begin{array}{c}1.83 \\
(1.15-8.05) \\
1.27-6.45\end{array}$ \\
\hline $\begin{array}{l}\text { Mouse } \\
\text { STR }^{b}\end{array}$ & $\begin{array}{l}\text { It data } \\
\text { Ire data }\end{array}$ & $\begin{array}{c}15.3 \\
(13.3-30.2) \\
23.8-100\end{array}$ & $\begin{array}{c}2.68 \\
(1.79-3.17) \\
1.04-2.10\end{array}$ & $\begin{array}{c}0.441 \\
(0.302-0.502) \\
0.100-2.60\end{array}$ & $\begin{array}{c}7.91 \\
(<\text { LOD-12.6) } \\
2-31.9\end{array}$ \\
\hline $\begin{array}{l}\text { Mouse } \\
\mathrm{CX}^{\mathrm{b}}\end{array}$ & $\begin{array}{l}\text { Current data } \\
\text { Literature data }\end{array}$ & $\begin{array}{c}14.6 \\
(14.4-19.5) \\
14-50\end{array}$ & $\begin{array}{c}2.09 \\
(1.74-2.66) \\
0.500-2.00\end{array}$ & $\begin{array}{c}0.138 \\
(<\mathrm{LOD}-0.300) \\
0.100-3.20\end{array}$ & $\begin{array}{c}<\mathrm{LOD} \\
(<\mathrm{LOD}-4.04) \\
1.5-7.72\end{array}$ \\
\hline $\begin{array}{l}\text { Mouse } \\
\mathrm{HC}^{\mathrm{b}}\end{array}$ & $\begin{array}{c}\text { Current data } \\
\text { Literature data }\end{array}$ & $\begin{array}{c}14 \\
(12.4-16.7) \\
14-30.7 \\
\end{array}$ & $\begin{array}{c}2.13 \\
(1.82-2.86) \\
0.55-3.90 \\
\end{array}$ & $\begin{array}{c}0.307 \\
(<\mathrm{LOD}-0.349) \\
0.070-3.10\end{array}$ & $\begin{array}{c}2.71 \\
(<\mathrm{LOD}-4.05) \\
1.2-7.66\end{array}$ \\
\hline $\begin{array}{l}\text { Mouse } \\
\text { plasma }^{\mathrm{a}}\end{array}$ & $\begin{array}{l}\text { Current data } \\
\text { Literature data }\end{array}$ & $\begin{array}{c}27.6 \\
(26.3-29.7) \\
22.2-100.3\end{array}$ & - & $\begin{array}{c}0.980 \\
(0.82-1.37) \\
0.54-1.12\end{array}$ & $\begin{array}{c}78.2 \\
(61.2-92.6) \\
29-301\end{array}$ \\
\hline $\begin{array}{c}\text { Rat } \\
\text { CNS** b }\end{array}$ & $\begin{array}{l}\text { Current data } \\
\text { Literature data }\end{array}$ & $\begin{array}{c}20.47 \\
(15.1-22.4) \\
12.16-74.4\end{array}$ & $\begin{array}{c}3.13 \\
(2.81-3.34) \\
0.722-6.00\end{array}$ & $\begin{array}{c}0.65 \\
(0.39-0.68) \\
0.4-3.7\end{array}$ & $\begin{array}{c}22.65 \\
(16.1-25.1) \\
1-101\end{array}$ \\
\hline $\begin{array}{c}\text { Rat } \\
\text { plasma }^{\text {a }}\end{array}$ & $\begin{array}{l}\text { Current data } \\
\text { Literature data }\end{array}$ & $\begin{array}{c}68.5 \\
(41.8-78.58) \\
14.4-84.02 \\
\end{array}$ & - & $\begin{array}{c}4.57 \\
(2.51-4.85) \\
0.6-2.55\end{array}$ & $\begin{array}{c}129 \\
(103-172) \\
40-90\end{array}$ \\
\hline
\end{tabular}

Current data are presented as median (IQR), whereas the whole range of median/mean values are presented in case of literature data.

${ }^{\mathrm{a}}$ For human and murine plasma and CSF samples, data are presented in $\mu \mathrm{M}$ for TRP and KYN, and nM for KYNA.

${ }^{\mathrm{b}}$ For murine brain samples, data are presented in nmol/g ww for TRP, 5-HT and KYN and pmol/g ww for KYNA

*The literature data was used from articles presented in Supplementary file Table S1 from (Cseh et al., 2019) and the related articles from (Sadok et al., 2017) were included. 
${ }^{* *}$ For rat CNS samples, pooled regions, including the brain and the upper part of the spinal cord containing the TNC, were used. 5-HT serotonin, CSF cerebrospinal fluid, IQR interquartile range, $K Y N$ kynurenine, $K Y N A$ kynurenic acid, $T N C$ trigeminal nucleus caudalis, TRP tryptophan. 


\section{Discussion}

\subsection{CFA model of orofacial pain}

Headache is one of the most common neurological disorders and it is one of the leading causes of health-related problems worldwide. In 2010, tension type headache and migraine were the second and third most prevalent conditions in the world, respectively, according to the Global Burden of Disease (GBD) study (Abraham et al., 2012; Saylor \& Steiner, 2018). Furthermore, the GBD study in 2015 established that headache is responsible (GBD 2015 DALYs and HALE Collaborators, 2016) for more disability adjusted life years than all other neurological disorders in combination. The treatment of primary headache disorders is challenging, requiring both acute and preventive therapeutic measures (Schuster \& Rapoport, 2016; American Headache Society, 2019). The preventive treatment aims at reducing the frequency, severity and duration of headaches, and to avoid medication-overuse headache. The efficacy of the currently applied drugs is not always satisfactory and the contraindications and side-effects often limit the options of the physician (Obermann et al., 2015; Diener et al., 2015). Therefore, there is a constant need to study and develop new molecules.

\section{Glutamate and migraine}

Peripheral and central sensitization manifest mainly in forms of hyperalgesia and allodynia. The activation of the peripheral terminals of the nociceptors is responsible for Glu release at central sites with the activation of ionotropic and metabotropic Glu receptors (Sarchielli et al., 2007). This process was demonstrated not only in preclinical studies (Bereiter \& Benetti, 1996; Oshinsky \& Luo, 2006; Lukács et al., 2017), but in migraine patients as well (Martínez et al., 1993; Peres et al., 2004). Accordingly, the role of glutamatergic pathways in association with different types of pain is well established (Osikowicz et al., 2013) and several antagonists of ionotropic glutamate receptors were investigated and found to be effective to decrease nociceptive transmission (Bleakman et al., 2006). However, they had severe side effects, and therefore, the interest in this direction of research diminished (Eide et al., 1995; JevtovicTodorovic et al., 1998). Nevertheless, ketamine, an NMDA receptor antagonist, is so far the only promising option in the treatment of severe or long-lasting migraine aura (Afridi et al., 2013), and tezampanel, which acts on the AMPA and kainate subtypes of ionotropic Glu receptors (Alt et al., 2006), has also shown promising results in acute migraine therapy (Sang et al., 2004). 


\section{Tryptophan metabolism and migraine}

It has been already demonstrated that the level of KYNA and some other KP metabolites are altered in migraine and cluster headache patients as well: there are significant reductions in the serum levels of KYN, KYNA, 3-hydroxy-kynurenine, 3HANA and quinolinic acid, whereas concentrations of TRP and anthranilic acid were significantly increased (Curto et al., 2015a, 2015b). KYNA as an endogenous NMDA receptor antagonist, is a molecule of interest for CNS drug development in case of several neurological conditions (Schwarcz, 2004), but due to its poor ability to cross the blood-brain barrier (BBB) and its rapid clearance from the body (Zádori et al., 2011a), its application for most CNS-related alterations is limited, and therefore, several KYNA analogs were synthetized (Szalardy et al., 2012; Vámos, 2012; Vécsei et al., 2013; Bohár et al., 2016). However, the first order neuron of pain processing is located outside the BBB (Messlinger \& Russo, 2019), so KYNA itself may have therapeutic potential as well. Accordingly, the antinociceptive properties of KYNA were proved in animal models of pain (Knyihár-Csillik et al., 2004; Tuboly et al., 2015). Furthermore, some of the developed analogs also displayed promising results in different animal models of headache (Knyihar-Csillik et al., 2008; Vámos et al., 2010; Park et al., 2011; Fejes-Szabó et al., 2014; Veres et al., 2017). In an earlier study we investigated two KYNA analogs where both of them proved to be effective in the formalin model of trigeminal pain (Veres et al., 2017). However, one of them was more effective than the other and according to our analyses the better performing compound caused a more pronounced elevation of KYNA concentration on the periphery, whereas in the CNS the concentrations of KYNA were similar. Based on these results we hypothesized that the peripheral elevation of KYNA may be enough to exert beneficial effects on pain processing and targeting this component could provide an option to pharmaceutical drug design without the obligation of good penetration through the BBB.

Elevated Glu concentration in the TNC of CFA-treated rats, demonstrated by the current study, is accompanied by increased KYN and KYNA levels, which may serve as a feedback mechanism to the sensitization process caused by Glu. This hypothesis is supported by the above-mentioned findings (Curto et al., 2015a, 2015b) that decreased KP metabolite levels are associated with those headache disorders, where increased NMDA receptor activation may play a crucial role. These results may have a great importance especially in light of the finding that the slightly, but not significantly elevated GABA level may not be enough to counterbalance the effects of increased Glu levels. With regard to 5-HT, its cortical elevation by $48 \mathrm{~h}$ may serve 
as a feedback inhibitory response as well to ameliorate the activation of the trigeminovascular pathway (Noseda et al., 2017).

The results of this study draw attention to the limited time interval for therapies targeting glutamatergic pathways as well, as based on our previous experiments, a clear shift to dominantly peptide-mediated pain processing can be seen even from $24 \mathrm{~h}$ after CFA application (Körtési et al., 2019). This time point corresponds to the onset of peripheral and central sensitization of the TS as well in this model (Imbe et al., 2001; Park et al., 2008; Kopach et al., 2012). At this stage, mainly novel antibody-based therapies may come into account (Bigal et al., 2015; Castle \& Robertson, 2018; Raffaelli \& Reuter, 2018; Vollesen et al., 2018). With regard to these novel therapies, the focus of attention is on monoclonal antibodies targeting the CGRP pathway for the prophylactic treatment of migraine. Currently, four of these antibodies are in clinical trials (eptinezumab, galcanezumab, fremanezumab, erenumab) with promising results. However, the cost of these therapies is considerably higher than that of acute phase treatments.

\subsection{Quantification of TRP, 5-HT, KYN and KYNA from different biological samples}

During the method validation, all the calculated parameters (LOD, LOQ, intra- and interassay precision, and recovery) were in line with literature data (FDA, 2001; International Conference on Harmonisation, 2005). Intra-day precision, expressed with CV\% was below 5\% in each case (Table 7). Inter-assay values were all below 15\%, the maximum limit recommended by guidelines (FDA, 2001; International Conference on Harmonisation, 2005), except that for the 5-HT in the mouse brain and rat CNS samples, where a decrease of $52 \%$ and $19 \%$ was observed, respectively. This bias is higher than the maximum recommended value, but it can be easily explained, as due to the heterogeneity of the bioanalytical studies (Bischoff et al., 2007), there are many cases where the official guideline (FDA, 2001) proposed limits may not be applicable. In case of the brain samples of the current study, the inter-assay precision measurements were done from the already homogenized samples, as we considered that brain sample regions cannot be divided into two homogenous parts compared to the supernatant samples. Therefore, the bias value draws attention to the necessity of brain homogenization right before the measurement in line with our currently applied laboratory practice. Accordingly, the freshly homogenized mouse brain and rat CNS samples show stable concentration values, all below the recommended $5 \%$, i.e., $4.25 \mathrm{CV} \%$, and $1.91 \mathrm{CV} \%$, respectively. During the recovery study, the values should be within $15 \%$ of the nominal value, except the LOQ spiked recovery values, which should not 
deviate by more than 20\%, as recommended by the official guidelines (FDA, 2001; International Conference on Harmonisation, 2005). Regarding metabolite concentration values all of them were presented to be in the ranges from literature data (Table 9). 


\section{Conclusion}

In our study of CFA model of orofacial model, we presented the assessment of small molecule changes in the TNC and ssCX following CFA treatment for the first time, confirming a dominant role of glutamate in early pain processing and a compensatory elevation of KYNA with anti-glutamatergic properties. The time interval for the intervention targeting the glutamatergic system is presumed to be limited to the first $24 \mathrm{~h}$. The results of our previous therapeutic studies with KYNA or with its analogs strongly support this theory.

Due to the presented importance of KYNA, we further optimized, validated and checked the applicability of the methods on six different biological matrices, including human plasma and CSF, mouse brain and plasma, and rat CNS and plasma samples. Only a small modification was needed to optimize the method for murine CNS samples and it became applicable for the assessment of all the 4 above mentioned metabolites, i.e., TRP, 5-HT, KYN and KYNA, along with two internal standards, one for each detector. 


\section{Acknowledgment}

First of all, I would like to express my infinite gratitude to my supervisor Dr. Dénes Zádori for his continuous support and patience, for guiding me well in the last four years, for supporting my research ideas, and put them into practice and for teaching me to define the research problems and to solve them. On a personal level, Dénes inspired me with his precise, passionate, and hardworking attitude, which proved to me throughout the years that nothing is impossible. Without a doubt, I am where I am because of his support.

Furthermore, I would like to thank Dr. Gábor Veres, for teaching me a lot of work-related techniques and secrets, and for his continuous support, for reading and correcting every sentence I wrote, every presentation I made, and for being there as a friend, every time I needed a good word.

I would also like to thank Prof. László Vécsei and Prof. Péter Klivényi for their excellent scientific guidance and continuous support of all my research activities.

From all my colleagues, I would like to especially thank to my hardworking friend, Helga Polyák, for being supportive and for doing all the animal experiments I needed, without hesitating. I would also like to thank to all my colleagues with whom I performed the research work, especially my laboratory colleague and 'HPLC-partner', Nikolett Nánási, and to Dr. Tamás Körtési, Dr. Zsuzsanna Bohár, Dr. Rita Maszlag-Török, Eleonóra Spekker and Diána Martos, for helping me with the laboratory work.

My thank you goes to Dr. Cecília Rajda and Dr. Levente Szalárdy, two inspiring researchers and doctors, with whom I got to do two beautiful projects; to Prof. Tamás Janáky and his PhD student, Ferenc Tömösi, and to Dr. Ferenc Zádor for letting me take part in their projects; to Dr. István Szatmári for providing us the KYNA analogs and the internal standard. Furthermore, I would like to say special thanks to Prof. János Tajti and Árpád Párdutz for their kind help in the thorough revision of pain- and headache-related parts of my thesis.

I would like to express my special gratitude to my mum and dad, for their continuous support throughout my studies since 2007, when I moved to Kolozsvár to follow my dreams; to my sister, Judit and brother in-law, Petru, for being my supportive friends.

Last but not least, I would like to thank to my partner in life and adventures, my very best and supportive friend and my husband Dr. Gábor Veréb, for his presence in my life, for believing me and for not letting me give up in the hard times. 
I would also like to acknowledge all the financial support, which was given by the grants GINOP-2.3.2-15-2016-00034, EFOP-3.6.1-16-2016-00008, UNKP-19-3 New National Excellence Program of the Ministry for Innovation and Technology and the EFOP-3.6.3VEKOP-16-2017-00009. 


\section{References}

Abraham J et al. (2012). Years lived with disability (YLDs) for 1160 sequelae of 289 diseases and injuries 1990-2010: a systematic analysis for the Global Burden of Disease Study 2010. Lancet 380, 2163-2196.

Aczél T, Kun J, Szőke É, Rauch T, Junttila S, Gyenesei A, Bölcskei K \& Helyes Z (2018). Transcriptional alterations in the trigeminal ganglia, nucleus and peripheral blood mononuclear cells in a rat orofacial pain model. Front Mol Neurosci 11, 219.

Afridi SK, Giffin NJ, Kaube H \& Goadsby PJ (2013). A randomized controlled trial of intranasal ketamine in migraine with prolonged aura. Neurology 80, 642-647.

Alt A, Weiss B, Ogden AM, Li X, Gleason SD, Calligaro DO, Bleakman D \& Witkin JM (2006). In vitro and in vivo studies in rats with LY293558 suggest AMPA/kainate receptor blockade as a novel potential mechanism for the therapeutic treatment of anxiety disorders. Psychopharmacology 185, 240-247.

American Headache Society (2019). The American Headache Society position statement on integrating new migraine treatmentsinto clinical practice. Headache 59, 1-18.

Anthony M, Hinterberger H \& Lance JW (1967). Plasma serotonin in migraine and stress. Arch Neurol 16, 544-552.

Beitz AJ (1982). The nuclei of origin of brainstem serotonergic projections to the rodent spinal trigeminal nucleus. Neurosci Lett 32, 223-228.

Benarroch EE (2011). CGRP: sensory neuropeptide with multiple neurologic implications. Neurology 77, 281-287.

Bereiter DA \& Benetti AP (1996). Excitatory amino release within spinal trigeminal nucleus after mustard oil injection into the temporomandibular joint region of the rat. Pain 67, 451459.

Bigal ME, Walter S \& Rapoport AM (2015). Therapeutic antibodies against CGRP or its receptor. Br J Clin Pharmacol 79, 886-895.

Birch PJ, Grossman CJ \& Hayes AG (1988). Kynurenate and FG9041 have both competitive and non-competitive antagonist actions at excitatory amino acid receptors. Eur J Pharmacol 151, 313-315.

Bischoff R, Hopfgartner G, Karnes HT, Lloyd DK, Phillips TM, Tsikas D \& Xu G (2007). Summary of a recent workshop/conference report on validation and implementation of 
bioanalytical methods: Implications on manuscript review in the Journal of Chromatography B. J Chromatogr B 860, 1-3.

Bleakman D, Alt A \& Nisenbaum ES (2006). Glutamate receptors and pain. Semin Cell Dev Biol 17, 592-604.

Bohár Zs, Párdutz Á \& Vécsei L (2016). Tryptophan catabolites and migraine. Curr Pharm Des 22, 1013-1021.

Bolay H \& Moskowitz MA (2002). Mechanisms of pain modulation in chronic syndromes. Neurology 59, 2-7.

Borsook D (2012). Neurological diseases and pain. Brain 135, 320-344.

Braz JM, Enquist LW \& Basbaum AI (2009). Inputs to serotonergic neurons revealed by conditional viral transneuronal tracing. J Comp Neurol 514, 145-160.

Brennan KC \& Pietrobon D (2018). A Systems Neuroscience approach to migraine. Neuron 97, 1004-1021.

Burstein R, Collins B \& Jakubowski M (2004). Defeating migraine pain with triptans: a race against the development of cutaneous allodynia. Ann Neurol 55, 19-26.

Burstein R, Yamamura H, Malick A \& Strassman AM (1998). Chemical stimulation of the intracranial dura induces enhanced responses to facial stimulation in brain stem trigeminal neurons. J Neurophysiol 79, 964-982.

Campos F, Sobrino T, Pérez-Mato M, Rodríguez-Osorio X, Leira R, Blanco M, Mirelman D \& Castillo J (2013). Glutamate oxaloacetate transaminase: a new key in the dysregulation of glutamate in migraine patients. Cephalalgia 33, 1148-1154.

Cananzi AR, D’Andrea G, Perini F, Zamberlan F \& Welch KM (1995). Platelet and plasma levels of glutamate and glutamine in migraine with and without aura. Cephalalgia 15, 132135.

Castle D \& Robertson NP (2018). Monoclonal antibodies for migraine: an update. J Neurol 265, 1491-1492.

Clarke AP, Jandik P, Rocklin RD, Liu Y \& Avdalovic N (1999). An integrated amperometry waveform for the direct, sensitive detection of amino acids and amino sugars following anion-exchange chromatography. Anal Chem 71, 2774-2781.

Cooper JDH, Ogden G, McIntosh J \& Turnell DC (1984). The stability of the ophthalaldehyde/2-mercaptoethanol derivatives of amino acids: An investigation using high- 
pressure liquid chromatography with a precolumn derivatization technique. Anal Biochem 142, 98-102.

Cseh EK, Veres G, Szentirmai M, Nánási N, Szatmári I, Fülöp F, Vécsei L \& Zádori D (2019). HPLC method for the assessment of tryptophan metabolism utilizing separate internal standard for each detector. Anal Biochem 574, 7-14.

Curto M, Lionetto L, Negro A, Capi M, Fazio F, Giamberardino MA, Simmaco M, Nicoletti F \& Martelletti P (2015a). Altered kynurenine pathway metabolites in serum of chronic migraine patients. J Headache Pain 17, 47.

Curto M, Lionetto L, Negro A, Capi M, Perugino F, Fazio F, Giamberardino MA, Simmaco M, Nicoletti F \& Martelletti P (2015b). Altered serum levels of kynurenine metabolites in patients affected by cluster headache. J Headache Pain 17, 27.

Dario MF, Freire TB, Pinto CAS de O, Prado MSA, Baby AR \& Velasco MVR (2017). Tryptophan and kynurenine determination in human hair by liquid chromatography. $J$ Chromatogr B 1065-1066, 59-62.

Dazzi C, Candiano G, Massazza S, Ponzetto A \& Varesio L (2001). New high-performance liquid chromatographic method for the detection of picolinic acid in biological fluids. $J$ Chromatogr B 751, 61-68.

Deen M, Christensen CE, Hougaard A, Hansen HD, Knudsen GM \& Ashina M (2017). Serotonergic mechanisms in the migraine brain - a systematic review. Cephalalgia 37, 251264.

Diener HC, Charles A, Goadsby PJ \& Holle D (2015). New therapeutic approaches for the prevention and treatment of migraine. Lancet Neurol 14, 1010-1022.

Dolan JW (2012). When should an internal standard be used? LC GC 30, 474-480.

Edvinsson L, Tajti J, Szalárdy L \& Vécsei L (2018). PACAP and its role in primary headaches. J Headache Pain 19, 21.

Eide K, Stubhaug A, Oye I \& Breivik H (1995). Continuous subcutaneous administration of the $\mathrm{N}$-methyl-D-aspartic acid (NMDA) receptor antagonist ketamine in the treatment of postherpetic neuralgia. Pain 61, 221-228.

Enna SJ \& McCarson KE (2006). The role of GABA in the mediation and perception of pain. Adv Pharmacol 54, 1-27. 
FDA (2001). Guidance for Industry, Bioanalytical Method Validation, US Department of Health and Human Services, Food and Drug Administration, Center for Drug Evaluation and Research (CEDER).

Fejes-Szabó A, Bohár Z, Vámos E, Nagy-Grócz G, Tar L, Veres G, Zádori D, Szentirmai M, Tajti J, Szatmári I, Fülöp F, Toldi J, Párdutz Á \& Vécsei L (2014). Pre-treatment with new kynurenic acid amide dose-dependently prevents the nitroglycerine-induced neuronal activation and sensitization in cervical part of trigemino-cervical complex. J Neural Transm (Vienna) 121, 725-738.

Fekkes D, Voskuilen-Kooyman A, Jankie R \& Huijmans J (2000). Precise analysis of primary amino acids in urine by an automated high-performance liquid chromatography method: comparison with ion-exchange chromatography. J Chromatogr B 744, 183-188.

Ferrari MD, Odink J, Bos KD, Malessy MJ \& Bruyn GW (1990). Neuroexcitatory plasma amino acids are elevated in migraine. Neurology 40, 1582-1586.

de Freitas Silva DM, Ferraz VP \& Ribeiro ÂM (2009). Improved high-performance liquid chromatographic method for GABA and glutamate determination in regions of the rodent brain. J Neurosci Methods 177, 289-293.

Furuyama T, Kiyama H, Sato K, Park HT, Maeno H, Takagi H \& Tohyama M (1993). Regionspecific expression of subunits of ionotropic glutamate receptors (AMPA-type, KA-type and NMDA receptors) in the rat spinal cord with special reference to nociception. Brain Res Mol 18, $141-151$.

Garland EL (2012). Pain processing in the human nervous system: A selective review of nociceptive and biobehavioral pathways. Prim Care 39, 561-571.

GBD 2015 DALYs and HALE Collaborators (2016). Global, regional, and national disabilityadjusted life-years (DALYs) for 315 diseases and injuries and healthy life expectancy (HALE), 1990-2015: a systematic analysis for the Global Burden of Disease Study 2015. Lancet 388, 1603-1658.

GBD 2016 Headache Collaborators (2018). Global, regional, and national burden of migraine and tension-type headache, 1990-2016: a systematic analysis for the Global Burden of Disease Study 2016. Lancet Neurol 17, 954-976.

GBD 2016 Neurology Collaborators (2019). Global, regional, and national burden of neurological disorders, 1990-2016: a systematic analysis for the Global Burden of Disease Study 2016. Lancet Neurol 18, 459-480. 
Goadsby PJ, Holland PR, Martins-Oliveira M, Hoffmann J, Schankin C \& Akerman S (2017). Pathophysiology of migraine: A disorder of sensory processing. Physiol Rev 97, 553-622.

Grant RS, Coggan SE \& Smythe GA (2009). The physiological action of picolinic acid in the human brain. Int J Tryptophan Res 2, 71-79.

Hervé C, Beyne P, Jamault H \& Delacoux E (1996). Determination of tryptophan and its kynurenine pathway metabolites in human serum by high-performance liquid chromatography with simultaneous ultraviolet and fluorimetric detection. J Chromatogr $B$ 675, $157-161$.

Imbe H, Iwata K, Zhou QQ, Zou S, Dubner R \& Ren K (2001). Orofacial deep and cutaneous tissue inflammation and trigeminal neuronal activation. Implications for persistent temporomandibular pain. Cells Tissues Organs 169, 238-247.

International Conference on Harmonisation (2005). ICH Topic Q2 (R1), Validation of analytical procedures: text and methodology.

Jasmin L, Rabkin SD, Granato A, Boudah A \& Ohara PT (2003). Analgesia and hyperalgesia from GABA-mediated modulation of the cerebral cortex. Nature 424, 316-320.

Jevtovic-Todorovic V, Wozniak DF, Powell S, Nardi A \& Olney JW (1998). Clonidine potentiates the neuropathic pain-relieving action of MK-801 while preventing its neurotoxic and hyperactivity side effects. Brain Res 781, 202-211.

Kekesi G, Joo G, Csullog E, Dobos I, Klimscha W, Toth K, Benedek G \& Horvath G (2002). The antinociceptive effect of intrathecal kynurenic acid and its interaction with endomorphin-1 in rats. Eur J of Pharmacoly 445, 93-96.

Kendall DA, Browner M \& Enna SJ (1982). Comparison of the antinociceptive effect of gamma-aminobutyric acid (GABA) agonists: evidence for a cholinergic involvement. $J$ Pharmacol Exp Ther 220, 482-487.

Kessler M, Terramani T, Lynch G \& Baudry M (1989). A glycine site associated with Nmethyl-D-aspartic acid receptors: characterization and identification of a new class of antagonists. J Neurochem 52, 1319-1328.

Kimball RW, Friedman AP \& Vallejo E (1960). Effect of serotonin in migraine patients. Neurology 10, 107-111.

Knyihár-Csillik E, Chadaide Z, Okuno E, Krisztin-Péva B, Toldi J, Varga C, Molnár A, Csillik B \& Vécsei L (2004). Kynurenine aminotransferase in the supratentorial dura mater of the rat: effect of stimulation of the trigeminal ganglion. Exp Neurol 186, 242-247. 
Knyihar-Csillik E, Mihaly A, Krisztin-Peva B, Robotka H, Szatmari I, Fulop F, Toldi J, Csillik B \& Vecsei L (2008). The kynurenate analog SZR-72 prevents the nitroglycerol-induced increase of c-fos immunoreactivity in the rat caudal trigeminal nucleus: comparative studies of the effects of SZR-72 and kynurenic acid. Neurosci Res 61, 429-432.

Kopach O, Viatchenko-Karpinski V, Belan P \& Voitenko N (2012). Development of inflammation-induced hyperalgesia and allodynia is associated with the upregulation of extrasynaptic AMPA receptors in tonically firing lamina II dorsal horn neurons. Front Physiol 3, 391.

Körtési T, Tuka B, Nyári A, Vécsei L \& Tajti J (2019). The effect of orofacial complete Freund's adjuvant treatment on the expression of migraine-related molecules. The Journal of Headache and Pain 20, 43.

Lam DK, Sessle BJ \& Hu JW (2009). Glutamate and capsaicin effects on trigeminal nociception I: Activation and peripheral sensitization of deep craniofacial nociceptive afferents. Brain Res 1251, 130-139.

Latremoliere A \& Woolf CJ (2009). Central sensitization: a generator of pain hypersensitivity by central neural plasticity. J Pain 10, 895-926.

Levy RA \& Proudfit HK (1977). The analgesic action of baclofen [beta-(4-chlorophenyl)gamma-aminobutyric acid]. J Pharmacol Exp Ther 202, 437-445.

Lukács M, Warfvinge K, Tajti J, Fülöp F, Toldi J, Vécsei L \& Edvinsson L (2017). Topical dura mater application of CFA induces enhanced expression of c-fos and glutamate in rat trigeminal nucleus caudalis: attenuated by KYNA derivate (SZR72). J Headache Pain; 18, 39.

Malan TP, Mata HP \& Porreca F (2002). Spinal GABA(A) and GABA(B) receptor pharmacology in a rat model of neuropathic pain. Anesthesiology 96, 1161-1167.

Markovics A, Kormos V, Gaszner B, Lashgarara A, Szoke E, Sandor K, Szabadfi K, Tuka B, Tajti J, Szolcsanyi J, Pinter E, Hashimoto H, Kun J, Reglodi D \& Helyes Z (2012). Pituitary adenylate cyclase-activating polypeptide plays a key role in nitroglycerol-induced trigeminovascular activation in mice. Neurobiol Dis 45, 633-644.

Martínez F, Castillo J, Rodríguez JR, Leira R \& Noya M (1993). Neuroexcitatory amino acid levels in plasma and cerebrospinal fluid during migraine attacks. Cephalalgia 13, 89-93.

Marvizón JCG, Pérez OA, Song B, Chen W, Bunnett NW, Grady EF \& Todd AJ (2007). Calcitonin receptor-like receptor and receptor activity modifying protein 1 in the rat dorsal 
horn: localization in glutamatergic presynaptic terminals containing opioids and adrenergic alpha2C receptors. Neuroscience 148, 250-265.

Mawatari KI, Iinuma F \& Watanabe M (1989). Fluorimetric determination of kynurenine in human serum by high-performance liquid chromatography coupled with post-column photochemical reaction with hydrogen peroxide. J Chromatogr B 488, 349-355.

Mecs L, Tuboly G, Nagy E, Benedek G \& Horvath G (2009). The peripheral antinociceptive effects of endomorphin-1 and kynurenic acid in the rat inflamed joint model. Anesth Analg 109, 1297-1304.

Messlinger K \& Russo AF (2019). Current understanding of trigeminal ganglion structure and function in headache. Cephalalgia 39, 1661-1674.

Michael-Titus A, Revest P \& Shortland P (2010). The nervous system (Second Edition). Systems of the body series. Churchill Livingstone. Elsevier. London.

Mitsuhashi S, Fukushima T, Kawai J, Tomiya M, Santa T, Imai K \& Toyo'oka T (2006). Improved method for the determination of kynurenic acid in rat plasma by column-switching HPLC with post-column fluorescence detection. Anal Chim Acta 562, 36-43.

Molnár-Perl I (2011). Advancement in the derivatizations of the amino groups with the ophthaldehyde-thiol and with the 9-fluorenylmethyloxycarbonyl chloride reagents. $J$ Chromatogr B 879, 1241-1269.

Murray CJL et al. (2012). Disability-adjusted life years (DALYs) for 291 diseases and injuries in 21 regions, 1990-2010: a systematic analysis for the Global Burden of Disease Study 2010. Lancet 380, 2197-2223.

Myint AM, Kim YK, Verkerk R, Scharpé S, Steinbusch H \& Leonard B (2007). Kynurenine pathway in major depression: evidence of impaired neuroprotection. $J$ Affect Disord 98, $143-151$.

Newcomer JW, Farber NB \& Olney JW (2000). NMDA receptor function, memory, and brain aging. Dialogues Clin Neurosci 2, 219-232.

Noseda R, Borsook D \& Burstein R (2017). Neuropeptides and Neurotransmitters That Modulate Thalamo-Cortical Pathways Relevant to Migraine Headache. Headache 57, $97-$ 111.

Noseda R \& Burstein R (2013). Migraine pathophysiology: anatomy of the trigeminovascular pathway and associated neurological symptoms, CSD, sensitization and modulation of pain. Pain; 154, 44-53 
Obermann M, Holle D, Naegel S, Burmeister J \& Diener H-C (2015). Pharmacotherapy options for cluster headache. Expert Opin Pharmacother 16, 1177-1184.

Oshinsky ML \& Luo J (2006). Neurochemistry of trigeminal activation in an animal model of migraine. Headache 46, S39-44.

Osikowicz M, Mika J \& Przewlocka B (2013). The glutamatergic system as a target for neuropathic pain relief. Experimental Physiology 98, 372-384.

Park JS, Yaster M, Guan X, Xu JT, Shih MH, Guan Y, Raja SN \& Tao YX (2008). Role of spinal cord alpha-amino-3-hydroxy-5-methyl-4-isoxazolepropionic acid receptors in complete Freund's adjuvant-induced inflammatory pain. Mol Pain 4, 67.

Park MK, Lee JH, Yang GY, Won KA, Kim MJ, Park YY, Bae YC \& Ahn DK (2011). Peripheral administration of NR2 antagonists attenuates orofacial formalin-induced nociceptive behavior in rats. Prog Neuropsychopharmacol Biol Psychiatry 35, 982-986.

Peres MFP, Zukerman E, Senne Soares CA, Alonso EO, Santos BFC \& Faulhaber MHW (2004). Cerebrospinal fluid glutamate levels in chronic migraine. Cephalalgia 24, 735-739.

Perucho J, Gonzalo-Gobernado R, Bazan E, Casarejos MJ, Jiménez-Escrig A, Asensio MJ \& Herranz AS (2015). Optimal excitation and emission wavelengths to analyze amino acids and optimize neurotransmitters quantification using precolumn OPA-derivatization by HPLC. Amino Acids 47, 963-973.

Pietrobon D \& Moskowitz M (2012). Pathophysiology of migraine. Annu Rev Physiol 75, 365391.

Polgár E, Hughes DI, Riddell JS, Maxwell DJ, Puskár Z \& Todd AJ (2003). Selective loss of spinal GABAergic or glycinergic neurons is not necessary for development of thermal hyperalgesia in the chronic constriction injury model of neuropathic pain. Pain 104, 229 239.

Polta JA \& Johnson DC (1983). The direct electrochemical detection of amino acids at a platinum electrode in an alkaline chromatographic effluent. J Liq Chromatogr 6, 1727-1743.

Quartu M, Serra MP, Ambu R, Lai ML \& Del Fiacco M (2002). AMPA-type glutamate receptor subunits $2 / 3$ in the human trigeminal sensory ganglion and subnucleus caudalis from prenatal ages to adulthood. Mech Ageing Dev 123, 463-471.

Raffaelli B \& Reuter U (2018). The biology of monoclonal antibodies: focus on calcitonin generelated peptide for prophylactic migraine therapy. Neurotherapeutics 15, 324-335.

Rizzoli P \& Mullally WJ (2018). Headache. Am J Med 131, 17-24. 
Sadok I, Gamian A \& Staniszewska MM (2017). Chromatographic analysis of tryptophan metabolites. J Sep Sci 40, 3020-3045.

Sahara Y, Noro N, Iida Y, Soma K \& Nakamura Y (1997). Glutamate receptor subunits GluR5 and KA-2 are coexpressed in rat trigeminal ganglion neurons. $J$ Neurosci 17, 6611-6620.

Sands SA, McCarson KE \& Enna SJ (2004). Relationship between the antinociceptive response to desipramine and changes in $\mathrm{GABAB}$ receptor function and subunit expression in the dorsal horn of the rat spinal cord. Biochem Pharmacol 67, 743-749.

Sang CN, Ramadan NM, Wallihan RG, Chappell AS, Freitag FG, Smith TR, Silberstein SD, Johnson KW, Phebus LA, Bleakman D, Ornstein PL, Arnold B, Tepper SJ \& Vandenhende F (2004). LY293558, a novel AMPA/GluR5 antagonist, is efficacious and well-tolerated in acute migraine. Cephalalgia 24, 596-602.

Sano M, Ferchaud-Roucher V, Nael C, Aguesse A, Poupeau G, Castellano B \& Darmaun D (2014). Simultaneous detection of stable isotope-labeled and unlabeled l-tryptophan and of its main metabolites, 1-kynurenine, serotonin and quinolinic acid, by gas chromatography/negative ion chemical ionization mass spectrometry. J Mass Spectrom 49, $128-135$.

Sarchielli P, Di Filippo M, Nardi K \& Calabresi P (2007). Sensitization, glutamate, and the link between migraine and fibromyalgia. Curr Pain Headache Rep 11, 343-351.

Saylor D \& Steiner TJ (2018). The Global Burden of Headache. Semin Neurol 38, 182-190.

Schuster NM \& Rapoport AM (2016). New strategies for the treatment and prevention of primary headache disorders. Nat Rev Neurol 12, 635-650.

Schwarcz R (2004). The kynurenine pathway of tryptophan degradation as a drug target. Curr Opin Pharmacol 4, 12-17.

Schwarcz R, Bruno JP, Muchowski PJ \& Wu HQ (2012). Kynurenines in the mammalian brain: when physiology meets pathology. Nat Rev Neurosci 13, 465-477.

Sicuteri F, Testi H \& Anselmi B (1961). Biochemical investigations in headache: increase in the hydroxyindoleacetic acid excretion during migraine attacks. Int Arch Allergy 19, 55-58. Silberstein SD (1994). Serotonin (5-HT) and migraine. Headache 34, 408-417.

Silberstein SD (2005). Chronic Daily Headache. J Am Osteopath Assoc 105, 23-29.

Soliman AC, Yu JSC \& Coderre TJ (2005). mGlu and NMDA receptor contributions to capsaicin-induced thermal and mechanical hypersensitivity. Neuropharmacology 48, 325332. 
Stragierowicz J, Daragó A, Brzeźnicki S \& Kilanowicz A (2017). Optimization of ultraperformance liquid chromatography (UPLC) with fluorescence detector (FLD) method for the quantitative determination of selected neurotransmitters in rat brain. $\operatorname{Med} \operatorname{Pr} \mathbf{6 8}, 583-$ 591.

Strimbu K \& Tavel JA (2010). What are Biomarkers? Curr Opin HIV AIDS 5, 463-466.

Syed AU, Koide M, Braas KM, May V \& Wellman GC (2012). Pituitary adenylate cyclaseactivating polypeptide (PACAP) potently dilates middle meningeal arteries: implications for migraine. J Mol Neurosci 48, 574-583.

Szalardy L, Zadori D, Toldi J, Fulop F, Klivenyi P \& Vecsei L (2012). Manipulating kynurenic acid levels in the brain - on the edge between neuroprotection and cognitive dysfunction. Curr Top Med Chem 12, 1797-1806.

Tajti J, Párdutz A, Vámos E, Tuka B, Kuris A, Bohár Z, Fejes A, Toldi J \& Vécsei L (2011). Migraine is a neuronal disease. J Neural Transm (Vienna) 118, 511-524.

Tallaksen-Greene SJ, Young AB, Penney JB \& Beitz AJ (1992). Excitatory amino acid binding sites in the trigeminal principal sensory and spinal trigeminal nuclei of the rat. Neurosci Lett 141, 79-83.

de Tommaso M, Arendt-Nielsen L, Defrin R, Kunz M, Pickering G \& Valeriani M (2016). Pain in Neurodegenerative Disease: Current Knowledge and Future Perspectives. Behav Neurol 2016; 7576292.

Tömösi F, Kecskeméti G, Cseh EK, Szabó E, Rajda C, Kormány R, Szabó Z, Vécsei L \& Janáky T (2020). A validated UHPLC-MS method for tryptophan metabolites: Application in the diagnosis of multiple sclerosis. J Pharml Biomed Anal 185, 113246.

Tuboly G, Tar L, Bohar Z, Safrany-Fark A, Petrovszki Z, Kekesi G, Vecsei L, Pardutz A \& Horvath G (2015). The inimitable kynurenic acid: the roles of different ionotropic receptors in the action of kynurenic acid at a spinal level. Brain Res Bull 112, 52-60.

Tuka B, Helyes Z, Markovics A, Bagoly T, Németh J, Márk L, Brubel R, Reglődi D, Párdutz A, Szolcsányi J, Vécsei L \& Tajti J (2012). Peripheral and central alterations of pituitary adenylate cyclase activating polypeptide-like immunoreactivity in the rat in response to activation of the trigeminovascular system. Peptides 33, 307-316.

Tuka B, Helyes Z, Markovics A, Bagoly T, Szolcsányi J, Szabó N, Tóth E, Kincses ZT, Vécsei L \& Tajti J (2013). Alterations in PACAP-38-like immunoreactivity in the plasma during ictal and interictal periods of migraine patients. Cephalalgia 33, 1085-1095. 
Vámos E (2012). Protective compounds in animal models of trigeminal activation and neurodegeneration. Ideggyogy $\mathrm{Sz}$ 65, 64-70.

Vámos E, Fejes A, Koch J, Tajti J, Fülöp F, Toldi J, Párdutz A \& Vécsei L (2010). Kynurenate derivative attenuates the nitroglycerin-induced CamKII $\alpha$ and CGRP expression changes. Headache 50, 834-843.

Vécsei L, Majláth Z, Balog A \& Tajti J (2015). Drug targets of migraine and neuropathy: treatment of hyperexcitability. CNS Neurol Disord Drug Targets 14, 664-676.

Vécsei L, Szalárdy L, Fülöp F \& Toldi J (2013). Kynurenines in the CNS: recent advances and new questions. Nat Rev Drug Discov 12, 64-82.

Veres G, Fejes-Szabó A, Zádori D, Nagy-Grócz G, László AM, Bajtai A, Mándity I, Szentirmai M, Bohár Z, Laborc K, Szatmári I, Fülöp F, Vécsei L \& Párdutz Á (2017). A comparative assessment of two kynurenic acid analogs in the formalin model of trigeminal activation: a behavioral, immunohistochemical and pharmacokinetic study. J Neural Transm (Vienna) 124, 99-112.

Veres G, Molnár M, Zádori D, Szentirmai M, Szalárdy L, Török R, Fazekas E, Ilisz I, Vécsei L \& Klivényi P (2015). Central nervous system-specific alterations in the tryptophan metabolism in the 3-nitropropionic acid model of Huntington's disease. Pharmacol, Biochem Behav 132, 115-124.

Veres G, Tellér A, Martos D, Szatmari I, Kiss L, Vécsei L \& Zádori D (2019). Determination of glutamate and GABA from rat central nervous system samples with HPLC utilizing fluorescent detection. Proceedings of the 25th International Symposium on Analytical and Environmental Problems. University of Szeged. Szeged. pp 368-372.

Vignau J, Jacquemont M-C, Lefort A, Imbenotte M \& Lhermitte M (2004). Simultaneous determination of tryptophan and kynurenine in serum by HPLC with UV and fluorescence detection. Biomed Chromatogr 18, 872-874.

Vollesen AL, Benemei S, Cortese F, Labastida-Ramírez A, Marchese F, Pellesi L, Romoli M, Ashina M \& Lampl C (2018). Migraine and cluster headache - the common link. J Headache Pain; 19, 89.

Watanabe Y, Yoshida R, Sono M \& Hayaishi O (1981). Immunohistochemical localization of indoleamine 2,3-dioxygenase in the argyrophilic cells of rabbit duodenum and thyroid gland. J Histochem Cytochem 29, 623-632. 
Werner ER, Bitterlich G, Fuchs D, Hausen A, Reibnegger G, Szabo G, Dierich MP \& Wachter H (1987). Human macrophages degrade tryptophan upon induction by interferon-gamma. Life Sci 41, 273-280.

Werner-Felmayer G, Werner ER, Fuchs D, Hausen A, Reibnegger G \& Wachter H (1989). Characteristics of interferon induced tryptophan metabolism in human cells in vitro. Biochim Biophys Acta 1012, 140-147.

WHO (2001). WHO International Programme on Chemical Safety Biomarkers in Risk Assessment: Validity and Validation. 2001.

World Health Organization and Lifting The Burden (2011). Atlas of headache disorders and resources in the world 2011.

Wu C \& Sun D (2015). GABA receptors in brain development, function, and injury. Metab Brain Dis 30, 367-379.

Xiao LD, Luo XB, Pi LG \& Tang AG (2008a). Simultaneous determination of kynurenine and kynurenic acid concentrations in human serum by HPLC with dual wavelengths fluorescence detection. Clin Chim Acta 395, 178-180.

Xiao Y, Richter JA \& Hurley JH (2008b). Release of glutamate and CGRP from trigeminal ganglion neurons: Role of calcium channels and 5-HT1 receptor signaling. Mol Pain 4, 12.

Zádori D, Ilisz I, Klivényi P, Szatmári I, Fülöp F, Toldi J, Vécsei L \& Péter A (2011a). Timecourse of kynurenic acid concentration in mouse serum following the administration of a novel kynurenic acid analog. J Pharm Biomed Anal 55, 540-543.

Zádori D, Klivényi P, Plangár I, Toldi J \& Vécsei L (2011b). Endogenous neuroprotection in chronic neurodegenerative disorders: with particular regard to the kynurenines. $\mathrm{J}$ Cell Mol Med 15, 701-717.

Zhang X, He Y \& Ding M (2009). Simultaneous determination of tryptophan and kynurenine in plasma samples of children patients with Kawasaki disease by high-performance liquid chromatography with programmed wavelength ultraviolet detection. J Chromatogr B 877, 1678-1682.

Zhao J (2013). Plasma kynurenic acid/tryptophan ratio: a sensitive and reliable biomarker for the assessment of renal function. Ren Fail 35, 648-653.

Zhao J, Chen H, Ni P, Xu B, Luo X, Zhan Y, Gao P \& Zhu D (2011). Simultaneous determination of urinary tryptophan, tryptophan-related metabolites and creatinine by high 
performance liquid chromatography with ultraviolet and fluorimetric detection. $J$ Chromatogr B 879, 2720-2725.

Zieglgänsberger W (2019). Substance P and pain chronicity. Cell Tissue Res 375, 227-241.

Zwakhalen SMG, Koopmans RTCM, Geels PJEM, Berger MPF \& Hamers JPH (2009). The prevalence of pain in nursing home residents with dementia measured using an observational pain scale. Eur J Pain 13, 89-93. 
$\int_{0}$ 


\title{
HPLC method for the assessment of tryptophan metabolism utilizing separate internal standard for each detector
}

\author{
Edina Katalin Cseh $^{\mathrm{a}}$, Gábor Veres ${ }^{\mathrm{a}, \mathrm{b}}$, Márton Szentirmai ${ }^{\mathrm{a}}$, Nikolett Nánási ${ }^{\mathrm{a}}$, István Szatmári ${ }^{\mathrm{c}}$, \\ Ferenc Fülöp ${ }^{c, d}$, László Vécsei ${ }^{\mathrm{a}, \mathrm{b}}$, Dénes Zádori ${ }^{\mathrm{a}, *}$ \\ ${ }^{a}$ Department of Neurology, Faculty of Medicine, Albert Szent-Györgyi Clinical Center, University of Szeged, Szeged, Hungary \\ ${ }^{\mathrm{b}}$ MTA-SZTE Neuroscience Research Group, Szeged, Hungary \\ ${ }^{\mathrm{c}}$ Institute of Pharmaceutical Chemistry, University of Szeged, Szeged, Hungary \\ ${ }^{\mathrm{d}}$ MTA-SZTE Stereochemistry Research Group, Szeged, Hungary
}

\section{A R T I C L E I N F O}

\section{Keywords:}

Tryptophan

Serotonin

Kynurenine pathway

High-performance liquid chromatography

Internal standard

\begin{abstract}
A B S T R A C T
The development of a validated method, applicable for the measurement of tryptophan (TRP) and serotonin (5HT), and that of the neuroprotective branch of the kynurenine pathway from several different biological matrices, including mouse brain, is described. Following the spectral analysis of the metabolites, they were quantified with reversed-phase high-performance liquid chromatography (HPLC), using separate internal standards (ISs) for UV (3-nitro-L-tyrosine) and fluorescent (the newly utilized 4-hydroxyquinazoline-2-carboxylic acid) detectors. With regard to validation parameters, selectivity, linearity, limit of detection, limit of quantification, precision and recovery were determined. Although the linearity ranges were different for the assessed matrices, the correlation coefficient was $>0.999$ in each case. Furthermore, good intra- and inter-day precision values were obtained with coefficient of variation $<5 \%$, and bias $<6.5 \%$ (except the 5-HT level in brain samples), respectively. The recoveries varied between $82.5 \%$ and $116 \%$. The currently developed methods yield opportunities for the assessment of concentration changes in the TRP metabolism from a wide range of biological matrices, therefore they may well be utilized in future clinical and preclinical studies, especially in view that so many metabolites with the application of ISs have not been detected from mouse brain with such a simple HPLC method before.
\end{abstract}

\section{Introduction}

Tryptophan (TRP), the essential amino acid obtained from diet, is mainly metabolized through the kynurenine (KYN) pathway (KP; Fig. 1), whereas only a small proportion of it is catabolized to the neurotransmitter serotonin (5-HT) [1]. Recently, a special attention has been paid to the KP in neuroscience research, especially in light of the well documented alterations of the pathway in numerous neurological disorders, such as Parkinson's disease, Alzheimer's disease, multiple sclerosis and schizophrenia [2-6]. Accordingly, several methods have been developed for the quantification of TRP and its metabolites, including those with multiple-step sample preparation, pre- or post- column derivatization or those needing complex instrumental background (e.g., high-performance liquid chromatography (HPLC) mass spectrometry, gas chromatography mass spectrometry) [7]. The assessment of TRP, KYN and kynurenic acid (KYNA), usually designated as the neuroprotective branch of the KP, may yield meaningful information in several preclinical and clinical studies [1]. The detection and quantification of these metabolites can be achieved in an easier way compared to the other compounds of the KP [7-9]. The partial assessment of the KP has been described with different HPLC methods, using several detection techniques, including UV detector (UVD) and fluorescence detector (FLD) $[9,10]$, diode array detector [11], electrochemical detector [12] or mass spectrometry [13,14], targeting

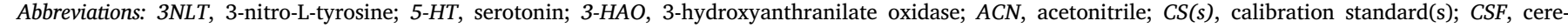

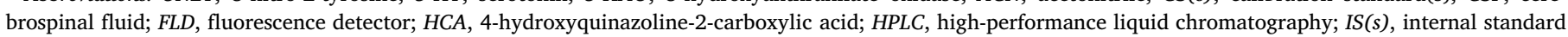

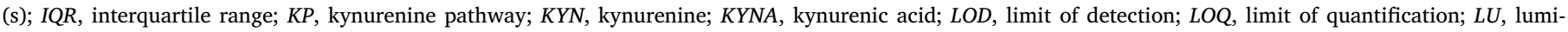

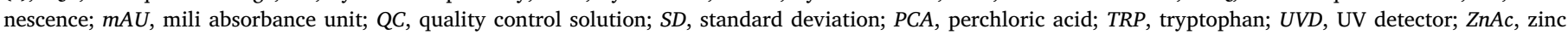
acetate; $W S$, working solution; $w w$, wet weight

* Corresponding author. Department of Neurology, Faculty of Medicine, Albert Szent-Györgyi Clinical Center, University of Szeged, Semmelweis street 6, H-6725, Szeged, Hungary.

E-mail address: zadori.denes@med.u-szeged.hu (D. Zádori).
} 


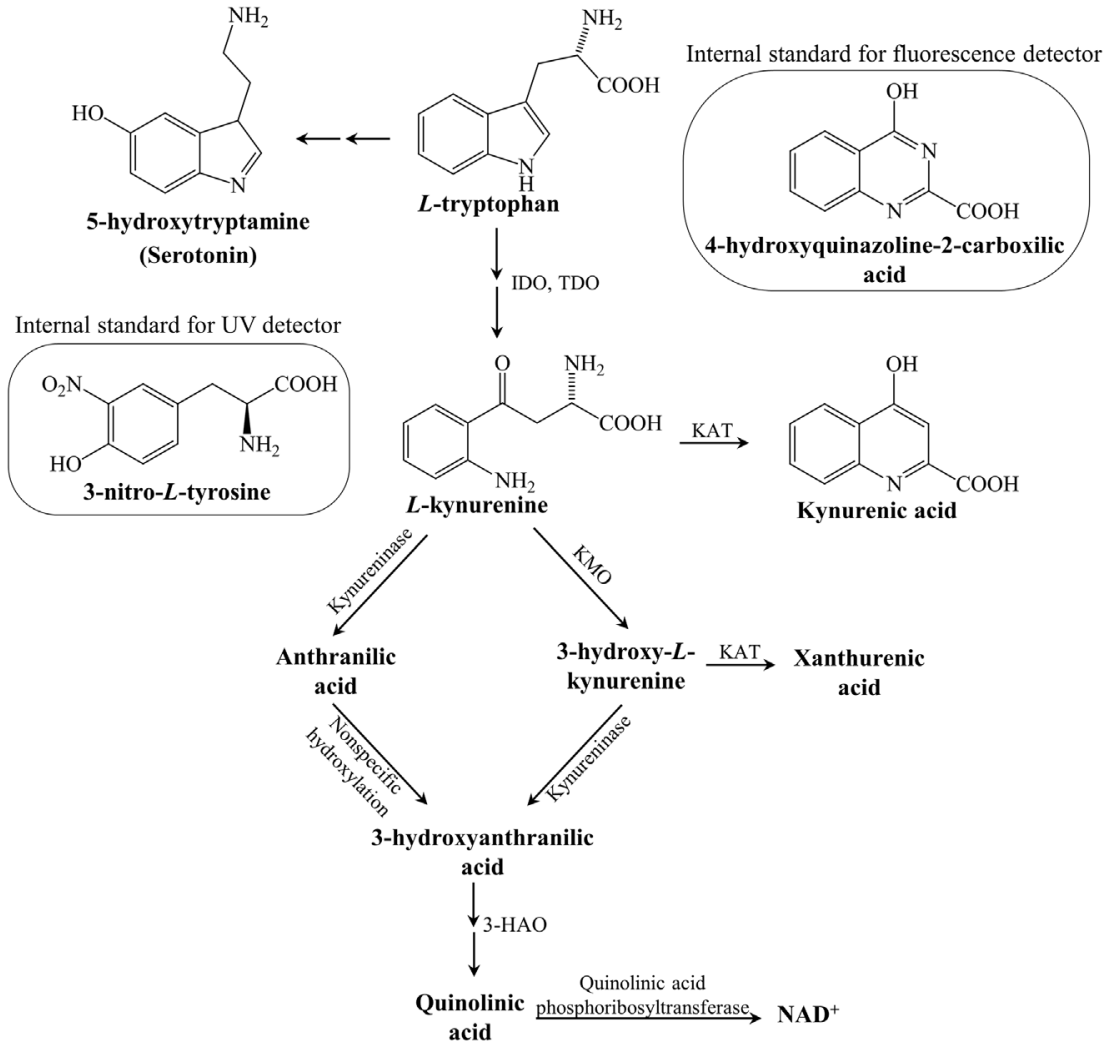

Fig. 1. The partial metabolism of tryptophan through the kynurenine and serotonin pathways and the structure of the two internal standards used for the UV and fluorescence detectors. 3-HAO 3-hydroxyanthranilate oxidase, IDO/TDO indoleamine 2,3-dioxygenase/tryptophan 2,3-dioxygenase, KAT kynurenine aminotransferase, $N A D^{+}$nicotineamide adenine dinucleotide. different biological matrices: human serum or plasma, and cerebrospinal fluid (CSF) [5,13-16], murine serum or plasma [9,14], and brain $[9,17]$. The heterogeneity of the methods is further increased by the application of internal standards (ISs), although only some (37.7\%) of the articles of interest (Supplementary file, Table S1) applied ISs at all, and none of them utilized ISs separately for each detector. This especially makes sense when in addition to the obviously necessary features of ISs (stability, pure form, absence in native sample, or no interference with another compound) [18,19], two often neglected characteristics are also taken into account: compatibility with the detector response and similarity in structure and properties with the analyzed compounds. Accordingly, the application of different ISs is required for each detector as the concentrations are calculated from a calibration plot where the concentration values are plotted against the response ratios. In light of these requirements, 3-nitro-L-tyrosine (3NLT) is not appropriate as an IS for the fluorescent detectable compounds TRP, 5-HT and KYNA. Consequently, the application of 4-hydroxyquinazoline-2-carboxylic acid (HCA) emerged as a new IS for the measurement of TRP, 5-HT and KYNA, with a special relevance to the latter one due to the similarities in their structure, which probably enables the detection of HCA at the same wavelength as KYNA, without affecting the running time of the sample. 3NLT has already been applied widely as an IS for UVD, as its structure is similar to that of KYN (Fig. 1).

The aim of the current study was to present a simple, rapid, precise, robust and economical method (95\% water in the mobile phase) for the simultaneous quantification of TRP, 5-HT (present in detectable amounts only in the mouse brain), KYN and KYNA by HPLC-UVD and FLD, using ISs for each detector (3NLT for the UVD, and HCA for the FLD), following a complete spectral analyses of each compound. To demonstrate the robustness of the method, the validation process was completed on four different biological matrices (mouse plasma and brain, human plasma and CSF) according to the ICH and FDA guidelines $[20,21]$. Furthermore, to verify the applicability of the currently developed methods, all of the metabolites of interest were quantified from the above-mentioned matrices, and the obtained concentration values were compared with the available literature data.

\section{Material and methods}

\subsection{Instrumentation and reagents}

The chromatographic analyses were performed using an Agilent 1100 HPLC system (Santa Clara, CA, USA) with Agilent G1314A UVD and G1321A FLD. The spectral analyses of the UV-detected compounds were made with an Agilent 8453 UV-Vis Spectroscopy System (Santa Clara, CA, USA). The reference compounds TRP, 5-HT, KYN, KYNA, 3NLT; perchloric acid (PCA), zinc acetate (ZnAc) and phosphoric acid were purchased from Sigma-Aldrich (Saint Louis, MO, USA). Acetonitrile (ACN) was obtained from Scharlau (Barcelona, Spain) and acetic acid from VWR International (Radnor, PA, USA). The di-sodiumhydrogen phosphate dihydrate was obtained from VWR International (Radnor, PA, USA) and potassium dihydrogen phosphate from Applichem Panreac (Darmstadt, Germany). The IS used for the FLD (HCA) was synthesized at the Department of Pharmaceutical Chemistry, University of Szeged, involving the ring closure of anthranilamide with diethyl oxalate, followed by the hydrolysis of the ester function [33].

\subsection{Collection and preparation of biological samples}

Mouse plasma samples and mouse brain tissues were obtained from 3 to 4 months old C57Bl/ 6 mice. The blood samples were collected into Na-EDTA-containing tubes and centrifuged at $3500 \mathrm{RPM}$ for $10 \mathrm{~min}$ and the resulting plasma samples were stored at $-80^{\circ} \mathrm{C}$ until analysis. The frozen plasma was thawed at room temperature, then deproteinized with $0.5 \mathrm{M}$ PCA solution $(1: 1 \mathrm{v} / \mathrm{v})$, containing both ISs at final concentration of $100 \mathrm{nM} \mathrm{HCA}$ and $2 \mu \mathrm{M}$ 3NLT, and centrifuged for $10 \mathrm{~min}$ at $12000 \mathrm{RPM}$ at $4{ }^{\circ} \mathrm{C}$. For the validation process, the individual samples were pooled, whereas for the demonstration of the applicability of the method and comparison of the obtained results with those 
Table 1

Linearity data, limit of detection and limit of quantification values for tryptophan, serotonin, kynurenine and kynurenic acid in different biological matrices.

\begin{tabular}{|c|c|c|c|c|c|}
\hline Biological sample & Analyte & Concentration range & Linear equation & LOD & LOQ \\
\hline \multirow[t]{3}{*}{ Human plasma } & $\mathrm{TRP}(\mu \mathrm{M})$ & $5-50$ & $y=0.194 x+461$ & 1.04 & 3.14 \\
\hline & $\mathrm{KYN}(\mu \mathrm{M})$ & $0.1-5$ & $y=0.00400 x+0.0871$ & 0.100 & 0.303 \\
\hline & KYNA (nM) & $2-100$ & $\mathrm{y}=7.17 \mathrm{x}-0.0357$ & 1.32 & 4.02 \\
\hline \multirow[t]{3}{*}{ Human CSF } & $\mathrm{TRP}(\mu \mathrm{M})$ & $0.1-5$ & $y=0.551 x-69.9$ & 0.102 & 0.308 \\
\hline & $\mathrm{KYN}(\mu \mathrm{M})$ & $0.05-3$ & $y=0.0117 x-0.201$ & 0.0274 & 0.0832 \\
\hline & KYNA (nM) & $2-60$ & $y=24.4 x-18.6$ & 1.23 & 3.72 \\
\hline \multirow{3}{*}{ Mouse plasma } & $\mathrm{TRP}(\mu \mathrm{M})$ & $1-30$ & $y=0.275 x-57.6$ & 0.557 & 1.69 \\
\hline & $\mathrm{KYN}(\mu \mathrm{M})$ & $0.05-3$ & $y=0.00452 x-0.147$ & 0.025 & 0.076 \\
\hline & KYNA (nM) & $2-100$ & $y=7.51 x-1.34$ & 1.33 & 4.03 \\
\hline \multirow[t]{4}{*}{ Mouse brain } & $\mathrm{TRP}(\mu \mathrm{M} ; \mathrm{nmol} / \mathrm{g}$ ww) & $0.2-10 ; 0.816-40.8$ & $y=1.63 x+2493$ & $0.204 ; 0.890$ & $0.619 ; 2.47$ \\
\hline & 5-HT (nM; pmol/g ww) & $25-1000 ; 102-4082$ & $\mathrm{y}=0.0821 \mathrm{x}+2.69$ & $8.55 ; 34.2$ & $25.9 ; 104$ \\
\hline & $\mathrm{KYN}(\mu \mathrm{M} ; \mathrm{nmol} / \mathrm{g}$ ww) & $0.1-3 ; 0.408-12.2$ & $y=0.0108 x-0.430$ & $0.0647 ; 0.259$ & $0.196 ; 0.785$ \\
\hline & KYNA (nM; pmol/g ww) & $0.5-60 ; 2-245$ & $y=14.8 x+7.12$ & $0.456 ; 1.82$ & $1.38 ; 5.50$ \\
\hline
\end{tabular}

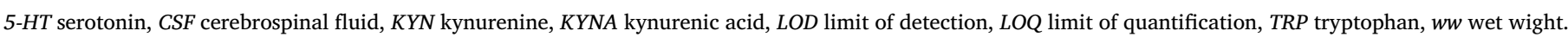

from the literature, the metabolites of interest were measured from 8 independent samples. Regarding the freshly prepared mouse brain samples, the tissues were weighed and then sonicated for $90 \mathrm{~s}$ in an icecooled solution, 1:5 w/v, comprising $0.5 \mathrm{M}$ PCA and the 2 ISs in an Eppendorf tube. The content of the Eppendorf tube was centrifuged for $10 \mathrm{~min}$ at $12000 \mathrm{RPM}$ at $4{ }^{\circ} \mathrm{C}$. For the validation process, pooled brain homogenates were applied, whereas the applicability of the method was tested on 8 independent striatum, cortex and hippocampus, obtained from the same mice as used for plasma sample measurements. The animal experiments were authorized by the local ethical committee of University of Szeged with adherence to the NIH guidelines and the EU directive 2010/63/EU for the protection of animals used for scientific purposes. Human plasma samples were obtained from 26 to 39 years old healthy subjects following obtaining written informed consent. Sample handling was almost the same as in case of mouse plasma samples, only the deproteinization process differed somewhat (the ratio of plasma and 0.5 M PCA solution was $1: 3 \mathrm{v} / \mathrm{v}$ ). The assessment of the applicability of the method was also carried out on 8 independent samples. The CSF samples were taken from 17 to 71 years old patients with headache who were initially suspected to have subarachnoid hemorrhage and underwent a spinal tap, but the CSF analysis was negative. Written informed consent was also obtained in each case. For the CSF samples, the same preparation procedure was applied as in cases of plasma samples, except using a dilution of $5: 6 \mathrm{v} / \mathrm{v}$. The applicability of the method was also tested on 8 independent CSF samples. All the human samples were obtained with the approval of the local Ethical Committee of the University of Szeged (46/2014), adhering to the tenets of the most recent revision of the Declaration of Helsinki.

\subsection{Chromatographic conditions}

Chromatographic separations were performed on a reversed-phase C18 column (Kinetex, $150 \times 4.6 \mathrm{~mm}$ I.D., $5 \mu \mathrm{m}$ particle size; Phenomenex Inc., Torrance, CA, USA) after passage through a precolumn (SecurityGuard, $4 \times 3.0 \mathrm{~mm}$ I.D., Phenomenex Inc., Torrance, CA, USA) with a mobile phase composition of $200 \mathrm{mM} \mathrm{ZnAc} \mathrm{solution} \mathrm{at}$ $\mathrm{pH}$ of 6.2 for plasma and CSF samples, and at $\mathrm{pH}$ of 5.8 for brain samples, adjusted with acetic acid. The organic component (ACN) in the mobile phase was $5 \%$, and the solution was filtered through a cellulose membrane with $0.2 \mu \mathrm{m}$ pore size. The flow rate was $1.2 \mathrm{ml} / \mathrm{min}$ and $20 \mu \mathrm{l}$ of the plasma supernatants were injected, whereas in case of CSF and brain homogenate the injection volume was $50 \mu 1$. The application of $\mathrm{ZnAc}$ at such high concentration as $200 \mathrm{nM}$ was necessary - focusing on the parallel prevention of precipitation as well - in light of the considerable increase of the fluorescence intensity of KYNA, which seems essential for its detection above limit of quantification (LOQ; Supplementary file, Fig. S3A). With the careful use of ZnAc at $200 \mathrm{nM}$, we did not experience precipitation and the lifespan of the applied column was not affected as well. The UV-Vis spectra data were collected from 200 to $800 \mathrm{~nm}$ in cases of KYN and 3NLT to determine the optimal wavelengths for measurements (Supplementary file Fig. S1).

The determination of optimal wavelengths in case of FLD was carried out via the collection of spectral data in the ranges of $220-380 \mathrm{~nm}$ (excitation) and $300-495 \mathrm{~nm}$ (emission) for each fluorescent compound, i.e., TRP, 5-HT, KYNA, and HCA (Supplementary file, Figs. S2A, B, C, D).

\subsection{Method validation}

The investigated validation parameters were selectivity, linearity, limit of detection (LOD), LOQ, precision and recovery, respecting the ICH [20] and FDA [21] guidelines. As insufficient amount of sample was obtained from one animal (especially in case of mouse plasma), following the recommendations provided by the FDA [21], pooled samples were used in each case, for constancy of validation process.

\subsubsection{Calibration curve and linearity}

Stock solutions with the concentration of $100 \mu \mathrm{M}$ were prepared by dissolving accurately weighed standard compounds in 0.5 M PCA solution, except the KYNA, which was dissolved in phosphorous buffer, with $\mathrm{pH}$ set at 6.2 with $85 \%$ phosphoric acid, due to solubility issues. A series of working solutions (WS) of the analytes with different concentration ranges for each matrix was prepared containing the ISs at final concentration of $2 \mu \mathrm{M}$ for 3NLT and $100 \mathrm{nM}$ for HCA. For the calibration curve, LOD and LOQ values, six calibration standards (CSs) were prepared by spiking the respective WSs into blank biological matrices, i.e., blank human plasma and CSF, and blank mouse plasma, respecting the dilution ratios presented above. With regard to mouse brain samples, the first step was the homogenization of the respective brain regions, and thereafter the supernatant was added the appropriate amount of the WSs. TRP, 5-HT, KYN and KYNA were prepared in the presented final concentration ranges (Table 1). The peak area response ratios were plotted against the corresponding concentration, and the linear regression computations were carried out by the least square method with the freely available $\mathrm{R}$ software ( $\mathrm{R}$ Development Core Team, 2002). The same procedure was applied for the preparation of the quality controls (QCs), i.e., spiking the blank biological matrices with the appropriate solutions, containing the analytes in three different concentration levels (low (LOQ), medium and high; Table 3) for performing the accuracy assays. Both CSs and QCs were prepared freshly, on the day of the measurements, whereas stock solutions and WSs were stored at $-80^{\circ} \mathrm{C}$. 
Table 2

Intra-assay (CV\%) and inter-assay (bias\%) coefficients for tryptophan, serotonin, kynurenine and kynurenic acid in the different biological matrices.

\begin{tabular}{lllllllll}
\hline Sample type & \multicolumn{2}{l}{ Tryptophan } & \multicolumn{2}{l}{ Serotonin } & \multicolumn{2}{l}{ Kynurenine } & \multicolumn{2}{c}{ Kynurenic acid } \\
\cline { 2 - 9 } & CV\% & Bias\% & CV\% & Bias\% & CV\% & Bias\% & CV\% & Bias\% \\
\hline Human plasma & 1.14 & 3.23 & - & - & 2.81 & 6.37 & 2.01 & 2.05 \\
Human CSF & 1.79 & 1.72 & - & - & 1.66 & 3.48 & 2.58 & 4.37 \\
Mouse plasma & 1.36 & 1.19 & - & - & 2.59 & 1.33 & 3.24 & 4.27 \\
Mouse brain & 2.41 & 1.11 & 4.25 & 52.4 & 2.32 & 6.16 & 3.42 & 3.79 \\
\hline
\end{tabular}

CSF cerebrospinal fluid, $C V$ coefficient of variation.

\section{Results and discussion}

\subsection{Selection of the excitation and emission wavelengths}

As a result of absorbance analyses for KYN and 3NLT (Supplementary file, Fig. S1) the wavelength of the UVD was set to $365 \mathrm{~nm}$ when $\mathrm{pH}$ was 6.2 , whereas a slight maximum absorbance shift was observed at $\mathrm{pH} 5.8$, therefore $360 \mathrm{~nm}$ was applied in this case. Following spectral analyses in case of fluorescent detection, the excitation and emission wavelengths at $\mathrm{pH} 6.2$ were set to 246 and $396 \mathrm{~nm}$ for the determination of KYNA and HCA, and to 220 and $410 \mathrm{~nm}$ for the determination of TRP, whereas at pH 5.8 the excitation and emission wavelengths were set to 239 and $400 \mathrm{~nm}$ for the determination of 5-HT, KYNA and HCA, and to 220 and $355 \mathrm{~nm}$ for the determination of TRP (Supplementary file, Fig. S2).

Table 3

Detailed recovery values of the compounds for three levels of concentration $(n=3)$ in different biological matrices.

\begin{tabular}{|c|c|c|c|c|c|}
\hline Sample type & Analyte & Conc. level of QCs & Added concentration & Measured concentration & Recovery (\%) \\
\hline \multirow[t]{12}{*}{ Human plasma } & \multirow[t]{4}{*}{$\mathrm{TRP}(\mu \mathrm{M})$} & Native & - & 43.5 & - \\
\hline & & Low (LOQ) & 3.14 & 47.1 & 116 \\
\hline & & Medium & 5 & 48.9 & 109 \\
\hline & & High & 15 & 60.6 & 114 \\
\hline & \multirow[t]{4}{*}{$\mathrm{KYN}(\mu \mathrm{M})$} & Native & - & 1.59 & - \\
\hline & & Low (LOQ) & 0.303 & 1.84 & 84.9 \\
\hline & & Medium & 0.5 & 2.06 & 95.1 \\
\hline & & High & 2 & 3.57 & 99.2 \\
\hline & \multirow[t]{4}{*}{ KYNA (nM) } & Native & - & 25.5 & - \\
\hline & & Low (LOQ) & 4.03 & 29.8 & 107 \\
\hline & & Medium & 10 & 34.9 & 94.3 \\
\hline & & High & 50 & 69.1 & 87.1 \\
\hline \multirow[t]{12}{*}{ Human CSF } & \multirow[t]{4}{*}{$\mathrm{TRP}(\mu \mathrm{M})$} & Native & - & 3.08 & - \\
\hline & & Low (LOQ) & 0.308 & 3.43 & 116 \\
\hline & & Medium & 1.2 & 4.71 & 96.5 \\
\hline & & High & 2.4 & 6.67 & 106 \\
\hline & \multirow{4}{*}{$\mathrm{KYN}(\mu \mathrm{M})$} & Native & - & 0.0893 & - \\
\hline & & Low (LOQ) & 0.0832 & 0.164 & 90.3 \\
\hline & & Medium & 0.4 & 0.436 & 86.7 \\
\hline & & High & 0.8 & 0.983 & 112 \\
\hline & \multirow[t]{4}{*}{ KYNA (nM) } & Native & - & 5.34 & - \\
\hline & & Low (LOQ) & 3.72 & 9.10 & 90.2 \\
\hline & & Medium & 10 & 15.9 & 86.7 \\
\hline & & High & 40 & 49.2 & 111.7 \\
\hline \multirow[t]{12}{*}{ Mouse plasma } & \multirow[t]{4}{*}{$\mathrm{TRP}(\mu \mathrm{M})$} & Native & - & 27.7 & - \\
\hline & & Low (LOQ) & 1.69 & 29.6 & 113 \\
\hline & & Medium & 5 & 32.9 & 103 \\
\hline & & High & 10 & 38.4 & 107 \\
\hline & \multirow[t]{4}{*}{$\mathrm{KYN}(\mu \mathrm{M})$} & Native & - & 0.844 & - \\
\hline & & Low (LOQ) & 0.075 & 0.906 & 82.5 \\
\hline & & Medium & 0.5 & 1.39 & 110 \\
\hline & & High & 2 & 2.85 & 100 \\
\hline & \multirow[t]{4}{*}{ KYNA (nM) } & Native & - & 72.5 & - \\
\hline & & Low (LOQ) & 4 & 76.6 & 104 \\
\hline & & Medium & 20 & 92.7 & 101 \\
\hline & & High & 50 & 130 & 115 \\
\hline \multirow[t]{16}{*}{ Mouse brain } & \multirow[t]{4}{*}{ TRP (nmol/g ww) } & Native & - & 11.1 & - \\
\hline & & Low (LOQ) & 2.47 & 13.7 & 107 \\
\hline & & Medium & 4.80 & 16.0 & 103 \\
\hline & & High & 9.60 & 21.4 & 108 \\
\hline & \multirow[t]{4}{*}{ 5-HT (pmol/g ww) } & Native & - & 3179 & - \\
\hline & & Low (LOQ) & 104 & 3295 & 111 \\
\hline & & Medium & 400 & 3386 & 105 \\
\hline & & High & 2000 & 5237 & 103 \\
\hline & \multirow[t]{4}{*}{ KYN (nmol/g ww) } & Native & - & 1.12 & - \\
\hline & & Low (LOQ) & 0.785 & 2.02 & 113 \\
\hline & & Medium & 1.60 & 2.91 & 111 \\
\hline & & High & 3.20 & 4.19 & 95.9 \\
\hline & \multirow[t]{4}{*}{ KYNA (pmol/g ww) } & Native & - & 5.51 & - \\
\hline & & Low (LOQ) & 5.50 & 10.9 & 97.8 \\
\hline & & Medium & 40 & 42 & 91.2 \\
\hline & & High & 160 & 155 & 93.8 \\
\hline
\end{tabular}

Conc. concentration, CSF cerebrospinal fluid, KYN kynurenine, KYNA kynurenic acid, LOQ limit of quantification, TRP tryptophan, $Q C$ quality control solution, ww wet weight. 

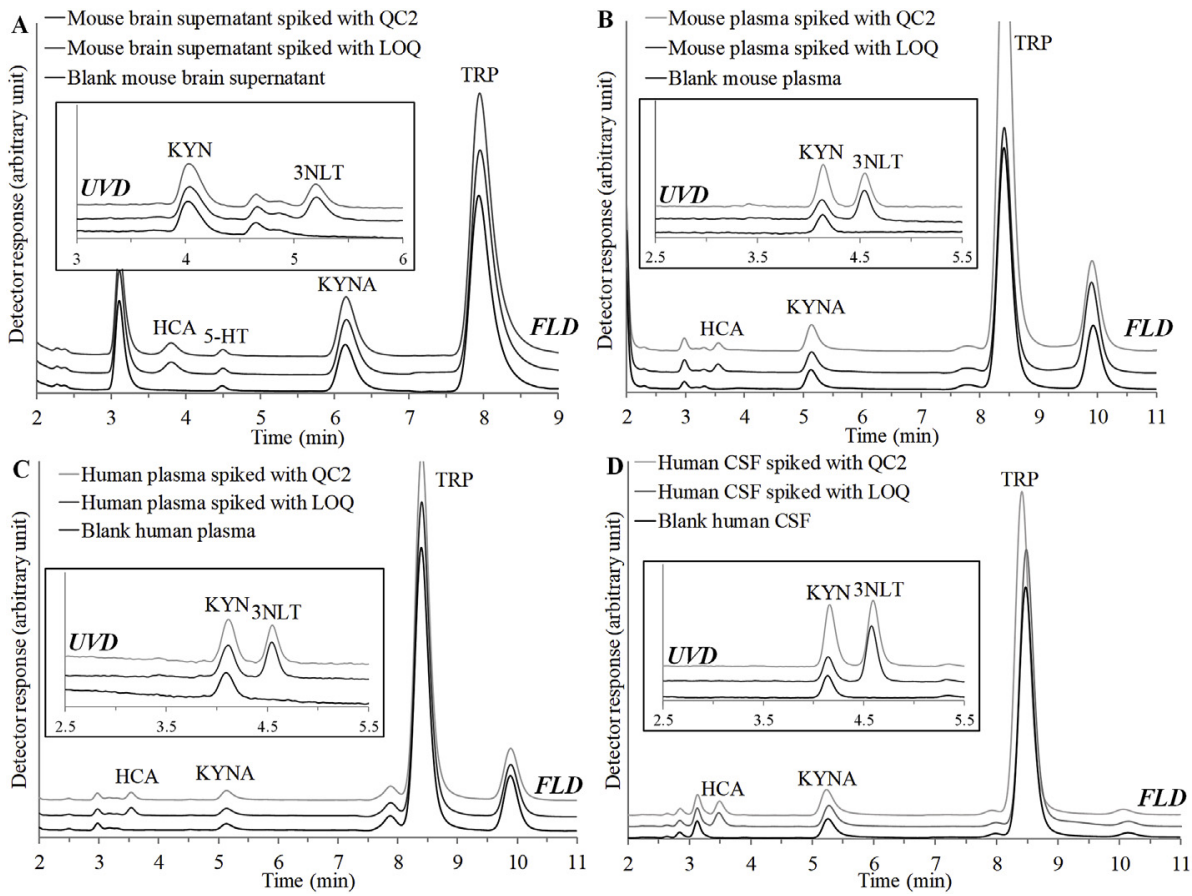

Fig. 2. Chromatograms of pooled mouse brain (A) and plasma (B), and human plasma (C) and cerebrospinal fluid (D) samples. UV chromatograms were obtained at $365 \mathrm{~nm} \mathrm{(B,C,D)} \mathrm{and} 360 \mathrm{~nm}$ (A), whereas for the fluorescence chromatograms, we applied Ex/Em.: 246/396 $\mathrm{nm}$ for the first $7 \mathrm{~min}$ and $220 / 410 \mathrm{~nm}$ for the remaining time (running time: 11 min) (B, C, D). For the brain samples (A) 239/ $400 \mathrm{~nm}$ and $220 / 335 \mathrm{~nm}$ were applied (running time: $9 \mathrm{~min}$ ). $3 N L T$ 3-nitro-L-tyrosine, 5-HT serotonin, CSF cerebrospinal fluid, FLD fluorescence detector, HCA 4-hydroxyquinazoline-2-carboxylic acid, $L O Q$ limit of quantification, $K Y N$ kynurenine, $K Y N A$ kynurenic acid, $Q C$ quality control solution, TRP tryptophan, UVD UV detector.

\subsection{Utilization of two internal standards and selectivity of the applied methods}

Several methods have already been published for the quantification of TRP and some of its metabolites (Supplementary file, Table S1), but from the published articles, only our research group reported the use of the 3NLT, when quantifying the analytes of interest from brain samples via the application of an Onyx Monolithic C18 column (100 mm $\times 4.6 \mathrm{~mm}$ I.D., Phenomenex Inc., Torrance, CA, USA) [9]. Indeed, the monolithic column provided a good running time $(7 \mathrm{~min}$ [9]), but the introduction of the novel IS (HCA) for the FLD led to coelution on the monolithic column (Supplementary file, Fig. S4) which resulted in the necessary change of the column. Accordingly, Kinetex C18 column was chosen with the aim of the parallel improvement of resolution. Although this novel setup with the optimization of flow rate and detection wavelengths was found to be suitable for measurements from plasma and CSF samples with appropriate selectivity and signal amplitude (Fig. 2), in case of mouse brain samples, an interfering peak was detected causing co-elution with both UVD and FLD (Fig. 3A and B). Accordingly, a further adjustment (i.e., the change of $\mathrm{pH}$ value of the mobile phase from 6.2 to 5.8, Fig. 3A and B) should be carried out to regain the appropriate selectivity (Supplementary file, Fig. S4). The further reduction of the $\mathrm{pH}$ considerably decreases the signal amplitude of KYNA, so it should be avoided (Supplementary file, Fig. S3B).

\subsection{Method validation}

\subsubsection{Linearity}

With regard to the ranges for external standards, it was kept in mind that under pathological or treatment conditions, a considerably large alteration may occur compared to the physiological values detected in different biological matrices. Accordingly, we tried to set up a relatively wide concentration range for external standards focusing at carrying out measurements with good linearity as well. The applied ranges
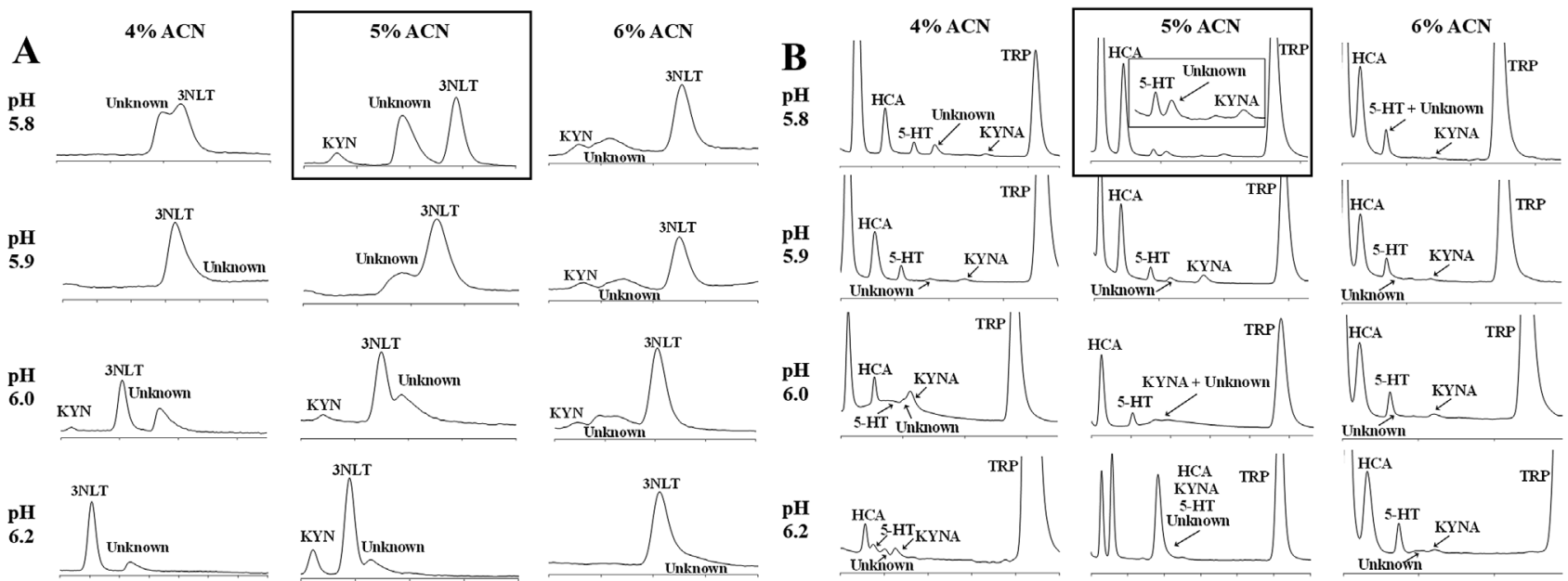

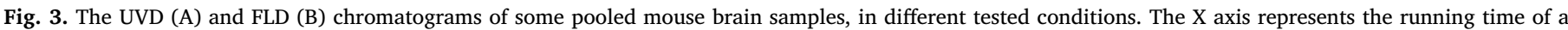

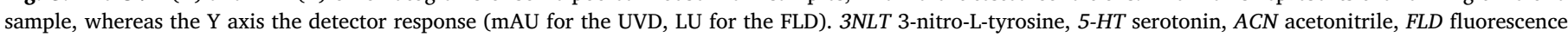

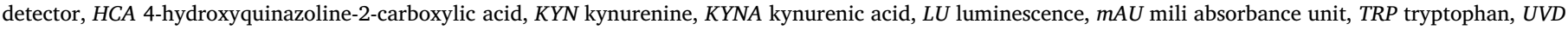
UV detector. 
Table 4

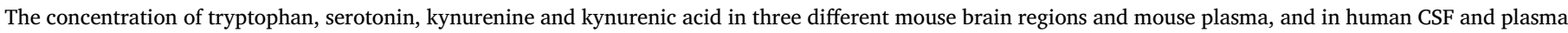
( $\mathrm{n}=8$ in each case).

\begin{tabular}{|c|c|c|c|c|c|c|}
\hline Biological sample & & TRP & 5-HT & KYN & KYNA & References \\
\hline \multirow[t]{2}{*}{ Mouse striatum $^{\mathrm{a}}$} & Current data & $15.3(13.3-30.2)$ & 2685 (1790-3173) & $0.441(0.302-0.502)$ & $7.91(<\mathrm{LOD}-12.6)$ & \\
\hline & Literature data & $23.8-100^{c}$ & $1040-2100^{c}$ & $0.100-2.60^{c}$ & $2-31.9^{c}$ & {$[9,24,35,36,39]$} \\
\hline \multirow[t]{2}{*}{ Mouse cortex ${ }^{a}$} & Current data & $14.6(14.4-19.5)$ & 2093 (1741-2675) & $0.138(<\mathrm{LOD}-0.300)$ & $<\operatorname{LOD}(<\mathrm{LOD}-4.04)$ & \\
\hline & Literature data & $14-50^{c}$ & $500-2000^{c}$ & $0.100-3.20^{c}$ & $1.5-7.72^{\mathrm{c}}$ & {$[9,23,34,35,37,38,40]$} \\
\hline \multirow[t]{2}{*}{ Mouse hippocampus $^{\mathrm{a}}$} & Current data & $14(12.4-16.7)$ & 2132 (1815-2857) & $0.307(<\mathrm{LOD}-0.349)$ & $2.71(<\mathrm{LOD}-4.05)$ & \\
\hline & Literature data & $14-30.7^{\mathrm{c}}$ & $550-3900^{c}$ & $0.070-3.10^{c}$ & $1.2-7.66^{\mathrm{c}}$ & {$[9,23,34-36,38,40]$} \\
\hline \multirow[t]{2}{*}{ Mouse plasma $^{\mathrm{b}}$} & Current data & $27.6(26.3-29.7)$ & - & $0.98(0.82-1.37)$ & $78.2(61.2-92.6)$ & \\
\hline & Literature data & $22.2-100.3^{c}$ & - & $0.54-1.12^{\mathrm{c}}$ & $29-301$ & [23-26] \\
\hline \multirow[t]{2}{*}{ Human $\mathrm{CSF}^{\mathrm{b}}$} & Current data & $2.72(2.04-3.31)$ & - & $0.0836(0.0586-0.109)$ & $1.83(1.15-8.05)$ & \\
\hline & Literature data & $0.16-2.52$ & - & $0.03-1.15$ & $1.27-6.45$ & {$[16,27,28]$} \\
\hline \multirow{2}{*}{ Human plasma ${ }^{\mathrm{b}}$} & Current data & 43.2 (36.7-49.7) & - & $2.14(1.90-2.35)$ & $26.8(23.9-32.9)$ & \\
\hline & Literature data & $34.8-71.8$ & - & $1.17-2.55$ & $13.8-140$ & {$[10,15,29-33]$} \\
\hline
\end{tabular}

Current data are presented as median (IQR), whereas the whole range of median/mean values are presented in case of literature data.

5-HT serotonin, CSF cerebrospinal fluid, IQR interquartile range, KYN kynurenine, KYNA kynurenic acid, $S D$ standard deviation TRP tryptophan.

${ }^{\text {a }}$ For mouse brain samples, data are presented in nmol/g ww for TRP and KYN, and pmol/g ww for 5-HT and KYNA.

b For human plasma and CSF samples, data are presented in $\mu \mathrm{M}$ for TRP and KYN, and nM for KYNA.

c In addition to literature data presented in Supplementary file Table S1, other references providing values of interest with different instrumental background (e.g., HPLC mass spectrometry) should also be included to be able to yield information for all the metabolites of interest.

mentioned in Table 1 were confirmed to be linear in all cases, with a correlation coefficient $>0.999$ for each compound when either FLD or UVD was applied.

\subsection{2. $L O D$ and $L O Q$}

LOD and LOQ were determined based on the guidelines [20,21] calculating by Equation (1), where $\sigma$ is the standard error of the intercept and $S$ ' is the slope of the calibration curve of the analyte, presented in Table 1.

$\mathrm{LOD}=3.3 \cdot \sigma / S^{\prime}$ and LOQ $=10 \cdot \sigma / S^{\prime}$

The obtained values were in line with literature data in each case (Supplementary file, Table S1).

\subsubsection{Precision}

The precision of the method was determined for each analyte in all matrices (Table 2). Intra-assay precision, expressed as CV\%, was evaluated by running six replicates, with values ranging between 1.14 and $4.25 \%$, i.e., all of them were below $5 \%$, in line with the values expected by the FDA [21]. Inter-assay precision was calculated by measuring the same samples used for the intra-assay precision with separate calibrations curves, after three days. The calculated values ranged between 1.11 and $6.37 \%$, except for the 5-HT in the mouse brain sample, where a decrease of $52 \%$ was observed. This bias is higher than the maximum recommended value (15\%) [21]. Due to the heterogeneity of the bioanalytical studies [22], there are many cases where the FDA proposed limits may not be applicable. In case of the brain samples of the current study, the inter-assay precision measurements were done from the already homogenized samples, as we considered that brain sample regions cannot be divided into two homogenous parts compared to the supernatant samples. Therefore, the bias value draws attention to the necessity of brain homogenization right before the measurement in line with our currently applied laboratory practice. Accordingly, the freshly homogenized brain samples show stable concentration values $(4.25 \mathrm{CV}$ $\%)$.

\subsubsection{Accuracy}

Recovery studies were performed using spiked samples at three different concentration levels (LOQ, medium and high), representing the entire range of values used for the calibration curve, with three replicates for each concentration. Recovery percentages were calculated as $\mathrm{R}=100 \times\left[\left(\mathrm{C}_{\mathrm{ss}}-\mathrm{C}_{\mathrm{ns}}\right) / \mathrm{C}_{\text {spike }}\right]$, where $\mathrm{C}_{\mathrm{ss}}$ is the concentration in the spiked homogenate sample, whereas $\mathrm{C}_{n s}$ is the concentration of the homogenate native sample (without spiking) and $\mathrm{C}_{\text {spike }}$ is the added concentration. The obtained values ranged between 82.5 and $116 \%$ (Table 3), which are within 15\% of the nominal value, except the LOQspiked recovery values, which did not deviate by more than $20 \%$, as recommended by the official guidelines [20,21].

\subsection{Application of the developed method on different biological matrices}

The results of the measurements of the metabolites of interest from the assessed biological matrices (mouse plasma and brain, human plasma and CSF) with the developed and validated method are summarized in Table 4. All the reported data in the current study are in line with those obtained from the scientific literature $[9,10,15,16,23-40]$.

\section{Conclusion}

In summary, in this paper we report an improved HPLC-UVD and FLD method for the quantification of TRP and some of its metabolites (5-HT, KYN, and KYNA). The novelty of this study is the utilization of two different ISs, a widely applied one for the UVD and a novel one for the FLD, proved to be adaptable for measurements from all the four different biological matrices. Although the developed method, suitable for measurements from mouse plasma and human plasma and cerebrospinal fluid was not appropriate for measurements from the mouse brain samples, the method could be further improved with slight modifications (changing the $\mathrm{pH}$ from 6.2 to 5.8 ) to become applicable for the assessment of all the 4 above-mentioned compounds from mouse brain samples as well with a single run, which has not been published before with HPLC-UVD and FLD (Supplementary file, Table S1). With regard to validation process, in addition to the achievement of appropriate selectivity, the linearity, LOD, LOQ, recovery and intra-assay precision values were all within the acceptable ranges provided by FDA and ICH $[20,21]$ and were in line with literature data proving the robustness of the method. The considerably high inter-assay value for 5HT draws attention to the necessity of brain homogenization right before the measurement. Moreover, to demonstrate the applicability of the developed method, the above-mentioned metabolites were quantified in different biological matrices and all of the reported concentration values were within or near the ranges obtained from the scientific literature. In conclusion, a fit-for-purpose, simple and economical method with the simultaneous application of two ISs was developed with one-step sample preparation, acceptable running time and with applicability in either human or animal model studies. 


\section{Declaration of interest}

The authors confirm that there were no conflicts of interest in performing this study.

\section{Acknowledgements}

This study was supported by the grants GINOP-2.3.2-15-201600034, EFOP-3.6.1-16-2016-00008, and National Brain Research Program 2017-1.2.1-NKP-2017-00002 NAP VI/4. Dénes Zádori was supported by the János Bolyai Research Scholarship of the Hungarian Academy of Sciences. Authors would like to thank to Diána Martos and Helga Polyák for providing the murine samples.

\section{Appendix A. Supplementary data}

Supplementary data to this article can be found online at https:// doi.org/10.1016/j.ab.2019.03.005.

\section{References}

[1] L. Vécsei, L. Szalárdy, F. Fülöp, J. Toldi, Kynurenines in the CNS: recent advances and new questions, Nat. Rev. Drug Discov. 12 (2013) 64-82 https://doi.org/10. 1038/nrd3793.

[2] D. Zádori, G. Veres, L. Szalárdy, P. Klivényi, L. Vécsei, Alzheimer's disease: recent concepts on the relation of mitochondrial disturbances, excitotoxicity, neuroinflammation, and kynurenines, J. Alzheimer's Dis. 62 (2018) 523-547 https://doi. org/10.3233/JAD-170929.

[3] D. Zádori, P. Klivényi, I. Plangár, J. Toldi, L. Vécsei, Endogenous neuroprotection in chronic neurodegenerative disorders: with particular regard to the kynurenines, J. Cell Mol. Med. 15 (2011) 701-717 https://doi.org/10.1111/j.1582-4934.2010. 01237.x.

[4] D. Zádori, G. Veres, L. Szalárdy, P. Klivényi, F. Fülöp, J. Toldi, L. Vécsei, Inhibitors of the kynurenine pathway as neurotherapeutics: a patent review (2012-2015), Expert Opin. Ther. Pat. 26 (2016) 815-832 https://doi.org/10.1080/13543776. 2016.1189531.

[5] H.P.G. Joaquim, A.C. Costa, W.F. Gattaz, L.L. Talib, Kynurenine is correlated with IL-1 $\beta$ in plasma of schizophrenia patients, J. Neural Transm. (2018) 1-5 https:// doi.org/10.1007/s00702-018-1838-8.

[6] K.H. Chang, M.L. Cheng, H.Y. Tang, C.Y. Huang, Y.R. Wu, C.-M. Chen, Alternations of metabolic profile and kynurenine metabolism in the plasma of Parkinson's disease, Mol. Neurobiol. (2018) 1-10 https://doi.org/10.1007/s12035-017-0845-3.

[7] I. Sadok, A. Gamian, M.M. Staniszewska, Chromatographic analysis of tryptophan metabolites, J. Sep. Sci. 40 (2017) 3020-3045 https://doi.org/10.1002/jssc. 201700184.

[8] H. Wolf, The effect of hormones and vitamin B6 on urinary excretion of metabolites of the kynurenine pathway, Scand. J. Clin. Lab. Invest. Suppl. 136 (1974) 1-186.

[9] G. Veres, M. Molnár, D. Zádori, M. Szentirmai, L. Szalárdy, R. Török, E. Fazekas, I. Ilisz, L. Vécsei, P. Klivényi, Central nervous system-specific alterations in the tryptophan metabolism in the 3-nitropropionic acid model of Huntington's disease, Pharmacol. Biochem. Behav. 132 (2015) 115-124 https://doi.org/10.1016/j.pbb. 2015.03.002.

[10] J. Zhao, H. Chen, P. Ni, B. Xu, X. Luo, Y. Zhan, P. Gao, D. Zhu, Simultaneous determination of urinary tryptophan, tryptophan-related metabolites and creatinine by high performance liquid chromatography with ultraviolet and fluorimetric detection, J. Chromatogr. B Analyt. Technol. Biomed. Life Sci. 879 (2011) 2720-2725 https://doi.org/10.1016/j.jchromb.2011.07.035.

[11] X. Zhang, Y. He, M. Ding, Simultaneous determination of tryptophan and kynurenine in plasma samples of children patients with Kawasaki disease by high-performance liquid chromatography with programmed wavelength ultraviolet detection, J. Chromatogr. B Analyt. Technol. Biomed. Life Sci. 877 (2009) 1678-1682 https://doi.org/10.1016/j.jchromb.2009.04.013.

[12] A. Vaarmann, A. Kask, U. Mäeorg, Novel and sensitive high-performance liquid chromatographic method based on electrochemical coulometric array detection for simultaneous determination of catecholamines, kynurenine and indole derivatives of tryptophan, J. Chromatogr. B Analyt. Technol. Biomed. Life Sci. 769 (2002) 145-153 https://doi.org/10.1016/S1570-0232(01)00639-0.

[13] W.H.A. de Jong, R. Smit, S.J.L. Bakker, E.G.E. de Vries, I.P. Kema, Plasma tryptophan, kynurenine and 3-hydroxykynurenine measurement using automated on-line solid-phase extraction HPLC-tandem mass spectrometry, J. Chromatogr. B Analyt. Technol. Biomed. Life Sci. 877 (2009) 603-609 https://doi.org/10.1016/j.jchromb. 2009.01.015.

[14] M. Möller, J.L. Du Preez, B.H. Harvey, Development and validation of a single analytical method for the determination of tryptophan, and its kynurenine metabolites in rat plasma, J. Chromatogr. B Analyt. Technol. Biomed. Life Sci. 898 (2012) 121-129 https://doi.org/10.1016/j.jchromb.2012.04.030.

[15] Bie Josien, Guest Jade, J. Guillemin Gilles, Grant Ross, Central kynurenine pathway shift with age in women, J. Neurochem. 136 (2016) 995-1003 https://doi.org/10. 1111/jnc. 13496.
[16] K.R. Linderholm, E. Skogh, S.K. Olsson, M.-L. Dahl, M. Holtze, G. Engberg, M. Samuelsson, S. Erhardt, Increased levels of kynurenine and kynurenic acid in the CSF of patients with schizophrenia, Schizophr. Bull. 38 (2012) $426-432$ https://doi. org/10.1093/schbul/sbq086.

[17] M.P. Heyes, B.J. Quearry, Quantification of 3-hydroxykynurenine in brain by high performance liquid chromatography and electrochemical detection, J. Chromatogr. 428 (1988) 340-344 https://doi.org/10.1016/S0378-4347(00)83925-0.

[18] J.W. Dolan, When should an internal standard be used? LC GC 30 (2012) 474-480.

[19] E. Lundanes, L. Reubsaet, T. Greibrokk, Chromatography: Basic Principles, Sample Preparations and Related Methods, John Wiley \& Sons, 2013.

[20] ICH Topic Q2 (R1), Validation of analytical procedures: text and methodology, Int Conf Harmon 1994 (2005) 1-17 November 1996.

[ 21] Guidance for Industry, Bioanalytical Method Validation, US Department of Health and Human Services, Food and Drug Administration, Center for Drug Evaluation and Research (CEDER), May 2001.

[22] R. Bischoff, G. Hopfgartner, H.T. Karnes, D.K. Lloyd, T.M. Phillips, D. Tsikas, G. Xu, Summary of a recent workshop/conference report on validation and implementation of bioanalytical methods: implications on manuscript review in the Journal of Chromatography B, J. Chromatogr. B 860 (2007) 1-3 https://doi.org/10.1016/j. jchromb.2007.10.039.

[23] R. Fuertig, A. Ceci, S.M. Camus, E. Bezard, A.H. Luippold, B. Hengerer, LC-MS/MSbased quantification of kynurenine metabolites, tryptophan, monoamines and neopterin in plasma, cerebrospinal fluid and brain, Bioanalysis 8 (2016) 1903-1917 https://doi.org/10.4155/bio-2016-0111.

[24] G. Mazarei, D.P. Budac, G. Lu, H. Adomat, E.S. Tomlinson Guns, T. Möller, B.R. Leavitt, Age-dependent alterations of the kynurenine pathway in the YAC128 mouse model of Huntington disease, J. Neurochem. 127 (2013) 852-867 https:// doi.org/10.1111/jnc.12350.

[25] S. Fujigaki, K. Saito, M. Takemura, N. Maekawa, Y. Yamada, H. Wada, M. Seishima, L-tryptophan-L-kynurenine pathway metabolism accelerated by toxoplasma gondi infection is abolished in gamma interferon-gene-deficient mice: cross-regulation between inducible nitric oxide synthase and indoleamine-2,3-dioxygenase, Infect. Immun. 70 (2002) 779-786 https://doi.org/10.1128/IAI.70.2.779-786.2002.

[26] P. Giménez-Gómez, M. Pérez-Hernández, M.D. Gutiérrez-López, R. Vidal, C. Abuin Martínez, E. O'Shea, M.I. Colado, Increasing kynurenine brain levels reduces ethanol consumption in mice by inhibiting dopamine release in nucleus accumbens, Neuropharmacology 135 (2018) 581-591 https://doi.org/10.1016/j.neuropharm. 2018.04.016.

[27] B. Widner, F. Leblhuber, D. Fuchs, Increased neopterin production and tryptophan degradation in advanced Parkinson's disease, J. Neural Transm. 109 (2002) 181-189 https://doi.org/10.1007/s007020200014.

[28] M.R. Keegan, S. Chittiprol, S.L. Letendre, A. Winston, D. Fuchs, A. Boasso, J. Iudicello, R.J. Ellis, Tryptophan metabolism and its relationship with depression and cognitive impairment among HIV-infected individuals, Int. J. Tryptophan Res. 9 (2016) 79-88 https://doi.org/10.4137/IJTR.S36464.

[29] N. Stoy, G.M. Mackay, C.M. Forrest, J. Christofides, M. Egerton, T.W. Stone, L.G. Darlington, Tryptophan metabolism and oxidative stress in patients with Huntington's disease, J. Neurochem. 93 (2005) 611-623 https://doi.org/10.1111/j. 1471-4159.2005.03070.x.

[30] G.M. Mackay, C.M. Forrest, N. Stoy, J. Christofides, M. Egerton, T.W. Stone, L.G. Darlington, Tryptophan metabolism and oxidative stress in patients with chronic brain injury, Eur. J. Neurol. 13 (2006) 30-42 https://doi.org/10.1111/j. 1468-1331.2006.01220.x

[31] A.A.B. Badawy, C.J. Morgan, Rapid isocratic liquid chromatographic separation and quantification of tryptophan and six kynurenine metabolites in biological samples with ultraviolet and fluorimetric detection, Int. J. Tryptophan Res. 3 (2010) 175-186 https://doi.org/10.4137/IJTR.S6225.

[32] E. Gulaj, K. Pawlak, B. Bien, D. Pawlak, Kynurenine and its metabolites in Alzheimer's disease patients, Adv. Med. Sci. 55 (2010) 204-211 https://doi.org/10 2478/v10039-010-0023-6

[33] J. Zhao, P. Gao, D. Zhu, Optimization of Zn2+-containing mobile phase for simultaneous determination of kynurenine, kynurenic acid and tryptophan in human plasma by high performance liquid chromatography, J. Chromatogr. B 878 (2010) 603-608 https://doi.org/10.1016/j.jchromb.2010.01.006.

[34] L.C. Souza, C.R. Jesse, L. Del Fabbro, M.G. de Gomes, N.S. Gomes, C.B. Filho, A.T.R. Goes, E.A. Wilhelm, C. Luchese, S.S. Roman, S.P. Boeira, Aging exacerbates cognitive and anxiety alterations induced by an intracerebroventricular injection of amyloid-ß1-42 peptide in mice, Mol. Cell. Neurosci. 88 (2018) 93-106 https://doi. org /10.1016/j.mcn.2018.01.005.

[35] A. Sekine, Y. Kuroki, T. Urata, N. Mori, T. Fukuwatari, Inhibition of large neutral amino acid transporters suppresses kynurenic acid production via inhibition of kynurenine uptake in rodent brain, Neurochem. Res. 41 (2016) 2256-2266 https:// doi.org/10.1007/s11064-016-1940-y.

[36] T.H. Kim, J. Choi, H.G. Kim, H.R. Kim, Quantification of neurotransmitters in mouse brain tissue by using liquid chromatography coupled electrospray tandem mass spectrometry, J. Anal. Methods in Chem. (2014), https://doi.org/10.1155/ 2014/506870.

[37] L. Cervo, A. Canetta, E. Calcagno, S. Burbassi, G. Sacchetti, S. Caccia, C. Fracasso, D. Albani, G. Forloni, R.W. Invernizzi, Genotype-dependent activity of tryptophan hydroxylase-2 determines the response to citalopram in a mouse model of depression, J. Neurosci. 25 (2005) 8165-8172 https://doi.org/10.1523/JNEUROSCI. 1816-05.2005.

[38] L.B. Devoino, E.L. Al'perina, E. Podgornaya, O.V. Polyakov, G.V. Idova, R.Y. Il'yuchenok, Brain content of 5-hydroxytryptamine and 5-hydroxyindoleacetic acid and immune response in aggressive C57Bl/6J mice, Bull. Exp. Biol. Med. 130 (2000) 954-956 https://doi.org/10.1023/A:1002853521450. 
[39] S.M. Gibney, B. McGuinness, C. Prendergast, A. Harkin, T.J. Connor, Poly I:C-induced activation of the immune response is accompanied by depression and anxiety-like behaviours, kynurenine pathway activation and reduced BDNF expression, Brain Behav. Immun. 28 (2013) 170-181 https://doi.org/10.1016/j.bbi.2012. 11.010
[40] S. Kelaï, T. Renoir, L. Chouchana, F. Saurini, N. Hanoun, M. Hamon, L. Lanfumey, Chronic voluntary ethanol intake hypersensitizes 5-HT(1A) autoreceptors in C57BL/6J mice, J. Neurochem. 107 (2008) 1660-1670 https://doi.org/10.1111/j. 1471-4159.2008.05733.x. 


\section{SUPPLEMENTARY FILE}

\section{HPLC method for the assessment of tryptophan metabolism utilizing separate internal standard for each detector}

Edina Katalin Cseh, Gábor Veres, Márton Szentirmai, Nikolett Nánási, István Szatmári, Ferenc Fülöp, László Vécsei, Dénes Zádori

\section{Spectral analyses of the investigated compounds}
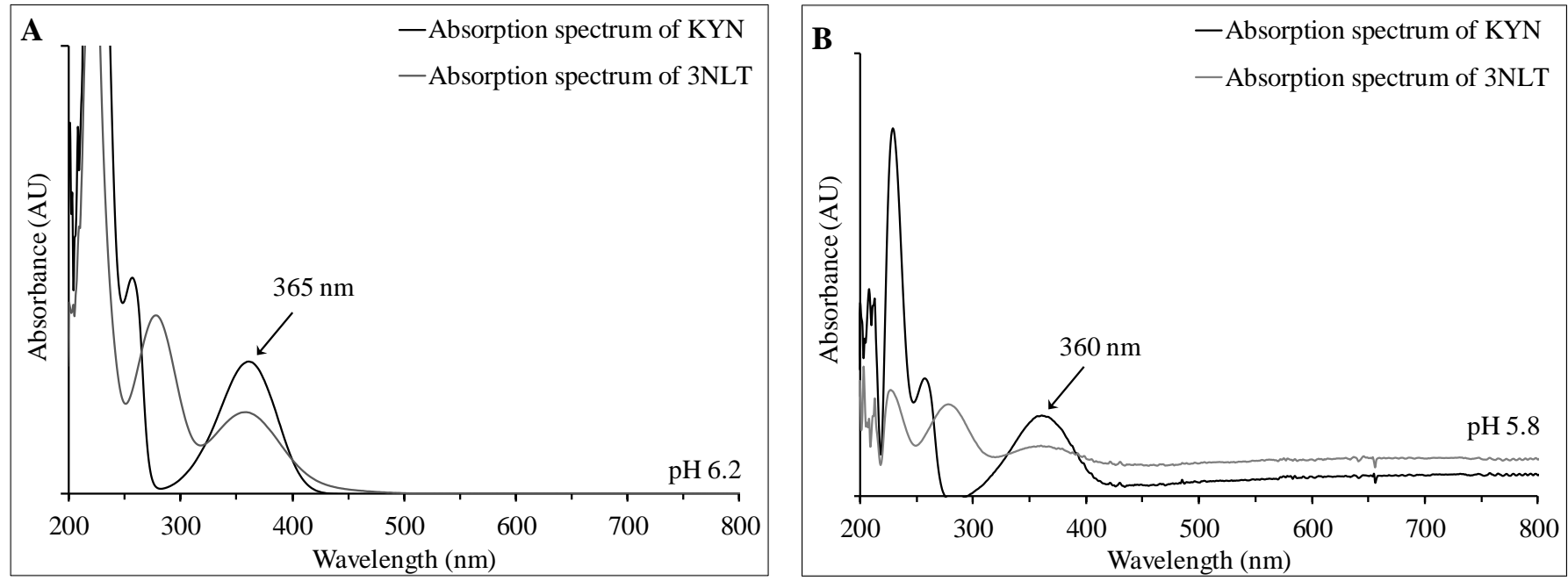

Fig. S1. Absorption spectral analyses of KYN and 3NLT, with mobile phase pH set at 6.2 (A) and 5.8 (B). 3NLT 3-nitro-L-tyrosine, $A U$ absorbance unit, $K Y N$ kynurenine 


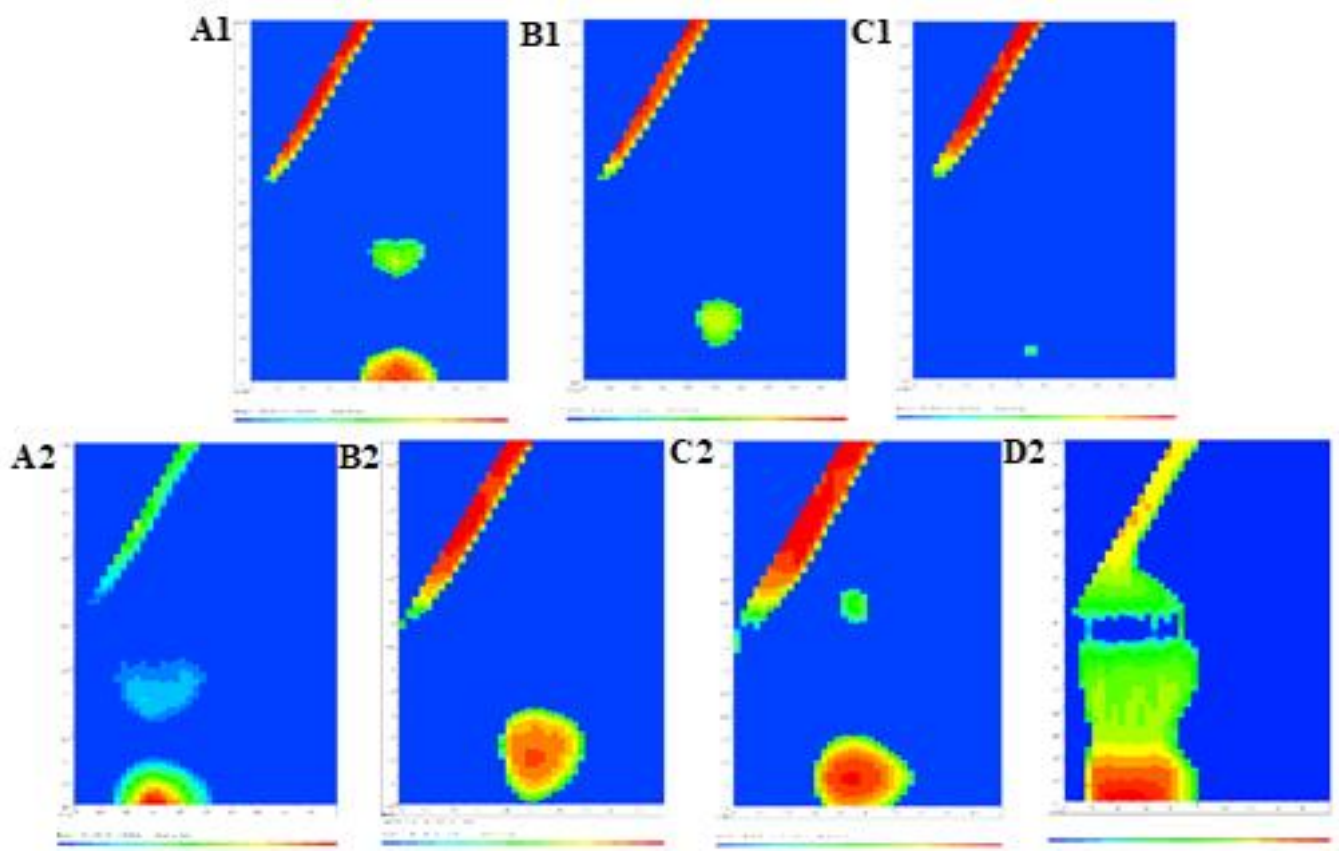

Fig. S2. Fluorescence 3D spectral scan (for both mobile phases: pH 6.2: A1, B1, C1 and pH 5.8 A2, B2, C2, D2) of the quantified compounds (TRP, A; KYNA, B; HCA, C; 5-HT, D). X axis represents the emission from 300 to $480 \mathrm{~nm}$, whereas y axis the excitation from 220 to $380 \mathrm{~nm}$. Colors represent the intensity of luminescence from 300 (dark blue) to different intensity values (red). 5-HT serotonin, HCA 4hydroxyquinolizone carboxylic acid, KYNA kynurenic acid, TRP tryptophan. 


\section{MOUSE BRAIN - METHOD DEVELOPMENT STEPS}
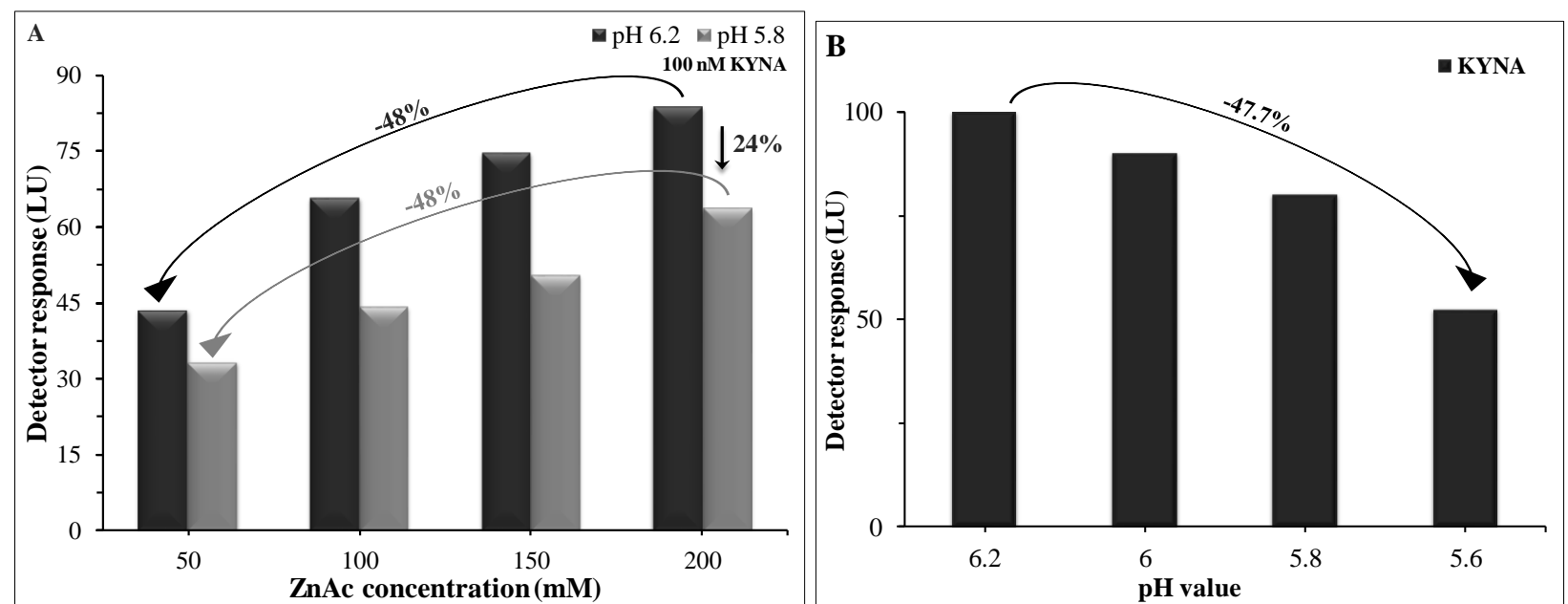

Fig. S3. The effect of ZnAc (A) and pH value (B) on the achieved signal intensity for KYNA. KYNA kynurenic acid, $L U$ luminescence, $Z n A c$ zinc acetate.

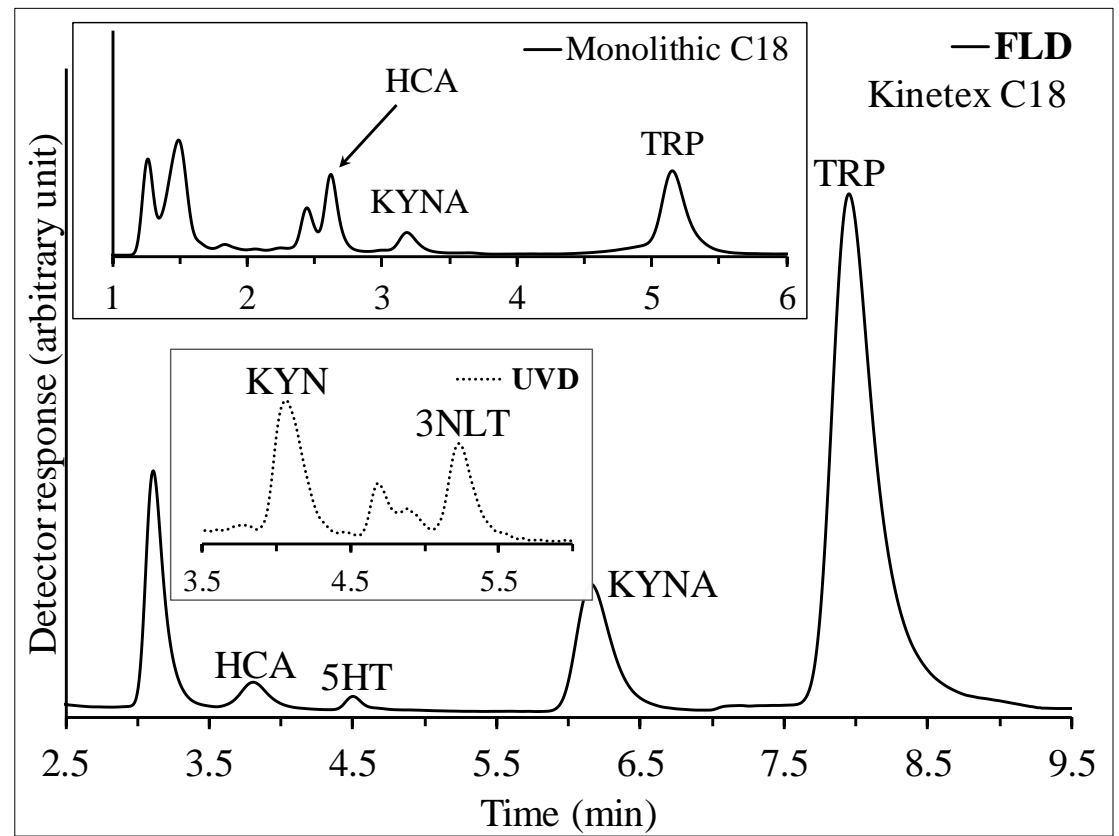

Fig. S4. The FLD chromatograms demonstrate the improved selectivity with the application of Kinetex C18 column compared to Onyx Monolithic C18 column. The UV chromatogram shows no interfering peaks affecting the selectivity. 


\section{Supplementary Tables}

Table S1. Reported data about internal standards used for the determination of TRP and some of its metabolites using both UVD and FLD ${ }^{\mathfrak{l}}$

\begin{tabular}{|c|c|c|c|c|c|c|c|c|}
\hline References & $\begin{array}{c}\text { Publications } \\
\text { by date }\end{array}$ & $\begin{array}{l}\text { Abs. det", } \\
\text { IS }\end{array}$ & FLD-IS & Analyzed metabolites & $\begin{array}{c}\text { Running time } \\
\text { (min) }\end{array}$ & $\begin{array}{c}\text { Spectral analyses of } \\
\text { compounds }\end{array}$ & $\begin{array}{c}\text { Method } \\
\text { validation }\end{array}$ & Sample type \\
\hline$[1]$ & $\begin{array}{l}\text { Werner et al. } \\
1987\end{array}$ & - & - & $\begin{array}{c}\text { TRP, KYN, AA // 3HAA } \\
\text { (AA - only in standard solution) }\end{array}$ & - & - & - & Various human cells \\
\hline$[2]$ & $\begin{array}{l}\text { Werner } \text { et al. } \\
1989\end{array}$ & $3 \mathrm{NLT}$ & - & $\begin{array}{c}\mathrm{TRP}, N F K, F A A, \mathrm{KYN}, 3 H A A, \mathrm{AA} \\
\text { (detectable only in one or two } \\
\text { cases) }\end{array}$ & - & - & - & Various human cells \\
\hline$[3]$ & $\begin{array}{l}\text { Herve et al. } \\
\quad 1996\end{array}$ & - & - & $\begin{array}{l}\text { TRP, KYN, KYNA, 3HAA, (3HK - } \\
\text { only in standard solution) }\end{array}$ & 20 & Yes (DAD and FLD) & - & Human serum \\
\hline$[4]$ & $\begin{array}{c}\text { Widner } \text { et al. } \\
1997\end{array}$ & $3 \mathrm{NLT}$ & - & TRP, KYN & 5 & - & - & Human serum \\
\hline$[5]$ & $\begin{array}{l}\text { Wu et al. } \\
2000\end{array}$ & - & - & KYN, KYNA & - & - & - & Rat brain and serum \\
\hline$[6]$ & $\begin{array}{l}\text { Dazzi et al. } \\
2001\end{array}$ & - & Me-TRP & $\begin{array}{c}\mathrm{TRP}, \mathrm{KYN}(N A, Q A, P A-\text { only in } \\
\text { standard solution) }\end{array}$ & 15 & Yes (DAD) & - & Human serum \\
\hline$[7]$ & $\begin{array}{c}\text { Pawlak et al. } \\
2001\end{array}$ & - & - & TRP, KYNA, AA // KYN, XA & - & Yes (DAD and FLD) & - & Rat serum \\
\hline$[8]$ & $\begin{array}{l}\text { Fujigaki et al. } \\
2002\end{array}$ & - & - & KYN, TRP & - & - & - & $\begin{array}{c}\text { Mouse brain, lung and } \\
\text { plasma }\end{array}$ \\
\hline$[9]$ & $\begin{array}{l}\text { Laich et al. } \\
\quad 2002\end{array}$ & $3 \mathrm{NLT}$ & - & TRP, KYN & 7 & - & - & Human serum \\
\hline$[10]$ & $\begin{array}{l}\text { Widner } \text { et al } \\
\quad 2002\end{array}$ & $3 \mathrm{NLT}$ & - & TRP, KYN & - & - & - & Human serum and CSF \\
\hline$[11]$ & $\begin{array}{c}\text { Pertovaraa et al. } \\
2005\end{array}$ & - & - & TRP // KYN & - & - & - & Human serum \\
\hline$[12]$ & $\begin{array}{l}\text { Vignau et al. } \\
2004\end{array}$ & - & Me-TRP & TRP, KYN & 10 & - & Yes & Human serum \\
\hline$[13]$ & $\begin{array}{l}\text { Stoy et al. } \\
\quad 2005\end{array}$ & $3 \mathrm{NLT}$ & - & TRP, KYN, KYNA & - & - & - & Human plasma \\
\hline$[14]$ & $\begin{array}{l}\text { Hwang et al. } \\
\quad 2005\end{array}$ & - & - & TRP, KYN & - & - & - & Dendritic cells \\
\hline$[15]$ & $\begin{array}{l}\text { Mackay et al. } \\
2006\end{array}$ & $3 N L T$ & - & TRP, KYN, KYNA & - & - & - & Human plasma \\
\hline$[16]$ & $\begin{array}{l}\text { Schröcksnadel } \\
\text { et al. } 2006\end{array}$ & $3 \mathrm{NLT}$ & - & TRP, KYN & 7 & - & - & Human serum \\
\hline$[17]$ & $\begin{array}{l}\text { Forrest et al. } \\
\quad 2007\end{array}$ & $3 \mathrm{NLT}$ & - & TRP, KYN, KYNA & - & - & - & Human plasma \\
\hline
\end{tabular}


Table S1. Reported data about internal standards used for the determination of TRP and some of its metabolites using both UVD and FLD ${ }^{£}$ (continued)

\begin{tabular}{|c|c|c|c|c|c|c|c|c|}
\hline References & $\begin{array}{c}\text { Publications } \\
\text { by date }\end{array}$ & $\begin{array}{l}\text { Abs. det*, } \\
\text { IS }\end{array}$ & FLD-IS & Analyzed metabolites & $\begin{array}{c}\text { Running time } \\
\text { (min) }\end{array}$ & $\begin{array}{c}\text { Spectral analyses of } \\
\text { compounds }\end{array}$ & $\begin{array}{c}\text { Method } \\
\text { validation }\end{array}$ & Biological matrices \\
\hline [18] & $\begin{array}{l}\text { Guillemin et al. } \\
2007\end{array}$ & - & - & TRP, KYN, KYNA & - & - & - & Human fetal brain \\
\hline$[19]$ & $\begin{array}{l}\text { Myint et al. } \\
2007\end{array}$ & - & $\begin{array}{l}\text { Norva- } \\
\text { line } 1 / /\end{array}$ & TRP // KYN, KYNA, 3HAA & - & - & - & Human plasma \\
\hline$[20]$ & $\begin{array}{l}\text { Badawy et al. } \\
2010\end{array}$ & - & - & $\begin{array}{c}\text { TRP, KYN, KYNA, 3HAA, XA, } \\
\text { 3HK (AA) }\end{array}$ & 13 & - & Yes, partially & $\begin{array}{l}\text { Human plasma, rat } \\
\text { serum and liver }\end{array}$ \\
\hline$[21]$ & $\begin{array}{l}\text { Gulaj et al. } \\
\quad 2010\end{array}$ & - & - & TRP, KYNA, AA// KYN // QUIN & - & Yes, partially & - & Human plasma \\
\hline$[22]$ & $\begin{array}{l}\text { Zhao et al. } \\
\quad 2010\end{array}$ & - & - & TRP, KYN, KYNA & 20 & - & Yes & Human plasma \\
\hline$[23]$ & $\begin{array}{l}\text { Oades et al. } \\
2010\end{array}$ & - & - & TRP, KYN, KYNA, 3HK, 5HIAA & - & - & - & Human serum \\
\hline [24] & $\begin{array}{l}\text { Krcmova et al. } \\
2011\end{array}$ & - & - & TRP, KYN, Creatine, NEO & 8 & - & Yes & Human serum \\
\hline$[25]$ & $\begin{array}{l}\text { Baran et al. } \\
\quad 2012\end{array}$ & - & - & KYN, KYNA & - & - & - & Human brain \\
\hline$[26]$ & $\begin{array}{l}\text { Linderholm et } \\
\quad \text { al. } 2012\end{array}$ & - & - & TRP, KYN, KYNA & $\sim 16-17$ & - & - & Human CSF \\
\hline$[27,28]$ & $\begin{array}{l}\text { Zhao et al. } \\
\text { 2011/2013 }\end{array}$ & Creatine $^{2}$ & - & TRP, KYN, KYNA / 5HIAA & 30 & - & - & $\begin{array}{c}\text { Human plasma and } \\
\text { urine }\end{array}$ \\
\hline [29] & $\begin{array}{l}\text { Lesniak et al. } \\
\quad 2013\end{array}$ & - & - & TRP, KYN, KYNA, 5-HT, 5HIAA & $\sim 33$ & $\begin{array}{c}\text { Yes (both UV-Vis and } \\
\text { FLD) }\end{array}$ & Yes & $\begin{array}{l}\text { Rabbit brain and } \\
\text { amniotic fluid }\end{array}$ \\
\hline$[30]$ & $\begin{array}{l}\text { Lim et al. } \\
\quad 2013\end{array}$ & - & - & TRP, KYN & - & - & - & Macaque macrophages \\
\hline$[31]$ & $\begin{array}{l}\text { Schwartz et al. } \\
2013\end{array}$ & - & - & TRP, KYN, KYNA, 3HK & $\sim 30$ & - & - & Human serum \\
\hline [32] & $\begin{array}{l}\text { Gibney et al. } \\
2013\end{array}$ & - & $\begin{array}{l}\mathrm{Me}- \\
5 \mathrm{HT}^{3}\end{array}$ & TRP, KYN & - & - & - & Mouse brain \\
\hline$[33]$ & $\begin{array}{l}\text { Krcmova et al. } \\
\quad 2015\end{array}$ & - & - & TRP, KYN, NEO & 6 & - & Yes & $\begin{array}{l}\text { Human amniotic fluid, } \\
\text { exudate and wounds }\end{array}$ \\
\hline
\end{tabular}


Table S1. Reported data about internal standards used for the determination of TRP and some of its metabolites using both UVD and FLD ${ }^{£}$ (continued)

\begin{tabular}{|c|c|c|c|c|c|c|c|c|}
\hline References & $\begin{array}{c}\text { Publications } \\
\text { by date }\end{array}$ & $\begin{array}{l}\text { Abs. det", } \\
\text { IS } \\
\end{array}$ & FLD-IS & Analyzed metabolites & $\begin{array}{c}\text { Running time } \\
\text { (min) }\end{array}$ & $\begin{array}{c}\text { Spectral analyses of } \\
\text { compounds }\end{array}$ & $\begin{array}{c}\text { Method } \\
\text { validation }\end{array}$ & Biological matrices \\
\hline [34] & $\begin{array}{l}\text { Wang et al } \\
\quad 2015\end{array}$ & - & - & TRP, KYN & 7 & - & $\begin{array}{c}\text { Partially: } \\
\text { precision only }\end{array}$ & $\begin{array}{c}\text { Murine bone marrow } \\
\text { mesenchymal stem } \\
\text { cells }\end{array}$ \\
\hline$[35]$ & $\begin{array}{c}\text { Sheipouri et al. } \\
2015\end{array}$ & - & - & TRP, KYN, KYNA & - & - & - & $\begin{array}{l}\text { Fibroblasts and } \\
\text { keratinocytes }\end{array}$ \\
\hline [36] & $\begin{array}{c}\text { Guloksuz et al. } \\
2015\end{array}$ & - & - & TRP, KYN, KYNA, 3HAA // 3HK & - & - & $\begin{array}{l}\text { Partially: only } \\
\text { for } 3 \mathrm{HK}\end{array}$ & Mouse serum \\
\hline$[37]$ & $\begin{array}{l}\text { Veres et.al } \\
\quad 2015\end{array}$ & $3 \mathrm{NLT}$ & - & TRP, KYN, KYNA & 7 & - & Yes & Mouse serum and brain \\
\hline$[38]$ & $\begin{array}{c}\text { Eminel et al. } \\
2016\end{array}$ & - & - & TRP, KYN, KYNA & - & - & - & PBMC culture \\
\hline [39] & $\begin{array}{c}\text { de Bie et al. } \\
2016\end{array}$ & - & - & TRP, KYN, KYNA & - & - & - & Human CSF \\
\hline$[40]$ & $\begin{array}{c}\text { Comai et al. } \\
2016\end{array}$ & - & - & TRP, 5HTP, 5-HT // KYN & - & - & - & Human serum \\
\hline [41] & $\begin{array}{l}\text { Keegan et al. } \\
2016\end{array}$ & $3 \mathrm{NLT}$ & - & TRP, KYN & - & - & $\begin{array}{l}\text { Partially: } \\
\text { within- and } \\
\text { between run }\end{array}$ & $\begin{array}{l}\text { Human plasma and } \\
\text { CSF }\end{array}$ \\
\hline [42] & $\begin{array}{c}\text { Sekine } \text { et al. } \\
2016\end{array}$ & & & TRP, KYN, KYNA & - & - & - & Mouse brain and serum \\
\hline [43] & $\begin{array}{l}\text { Jusof et al. } \\
\quad 2017\end{array}$ & - & - & TRP, KYN & 15 & - & - & $\begin{array}{c}\text { Murine liver and } \\
\text { plasma }\end{array}$ \\
\hline [44] & $\begin{array}{l}\text { Kubo et al. } \\
\quad 2017\end{array}$ & - & - & TRP, KYN, KYNA, AA & & - & - & Mouse serum \\
\hline$[45]$ & $\begin{array}{l}\text { Dario et al. } \\
\quad 2017\end{array}$ & - & Me-TRP & KYN, TRP & $\sim 9$ & - & Yes & Human hair \\
\hline [46] & $\begin{array}{l}\text { O'Farrel et al. } \\
2017\end{array}$ & - & $\begin{array}{l}\mathrm{Me}- \\
5 \mathrm{HT}^{3}\end{array}$ & TRP, KYN, KYNA & 18 & - & - & $\begin{array}{l}\text { Conditioned media of } \\
\text { IFN } \gamma \text {-stimulated BV-2 } \\
\text { microglia }\end{array}$ \\
\hline$[47]$ & $\begin{array}{c}\text { Oliveros et al. } \\
2017\end{array}$ & - & - & KYN, KYNA & $\sim 8$ & - & - & Mouse brain \\
\hline$[48]$ & $\begin{array}{c}\text { Bartosiewicz et } \\
\text { al. } 2017\end{array}$ & - & - & TRP, KYN, KYNA, AA & - & Yes, partially & - & Rat plasma \\
\hline [49] & $\begin{array}{l}\text { Tufvesson- Alm } \\
\text { et al. } 2018\end{array}$ & - & - & KYN, KYNA & $\sim 8$ & - & - & Mouse brain \\
\hline
\end{tabular}


Table S1. Reported data about internal standards used for the determination of TRP and some of its metabolites using both UVD and FLD ${ }^{£}$ (continued)

\begin{tabular}{|c|c|c|c|c|c|c|c|c|}
\hline References & $\begin{array}{c}\text { Publications } \\
\text { by date }\end{array}$ & $\begin{array}{l}\text { Abs. det*, } \\
\text { IS }\end{array}$ & FLD-IS & Analyzed metabolites & $\begin{array}{c}\text { Running time } \\
\text { (min) }\end{array}$ & $\begin{array}{c}\text { Spectral analyses of } \\
\text { compounds }\end{array}$ & $\begin{array}{c}\text { Method } \\
\text { validation }\end{array}$ & Biological matrices \\
\hline$[50]$ & $\begin{array}{l}\text { Michels et al. } \\
2018\end{array}$ & $3 \mathrm{NLT}$ & - & TRP. KYN, KYNA & 30 & - & - & Human serum \\
\hline [51] & $\begin{array}{c}\text { Bochinarz et al. } \\
2018\end{array}$ & - & - & TRP, KYN, KYNA & - & - & - & Cow serum and milk \\
\hline$[52]$ & $\begin{array}{c}\text { O`Rourke et al. } \\
2018\end{array}$ & $3 \mathrm{NLT}$ & - & TRP, KYN, KYNA & 30 & - & - & Breast milk \\
\hline$[53]$ & $\begin{array}{c}\text { Giménez- } \\
\text { Gómez et al. } \\
2018\end{array}$ & - & - & KYN// KYNA & - & - & - & $\begin{array}{c}\text { Mouse brain and } \\
\text { plasma }\end{array}$ \\
\hline
\end{tabular}

*Absorbance detector, including UV, DAD and PDA.

${ }^{£}$ Some of the methods utilized electrochemical detector, the compounds detected by it are not presented on the Analyzed metabolites row

"two different methods (i.e., column change AND/OR mobile phase change) were used

${ }^{1}$ Norvaline; IUPAC NAME: (2S)-2-aminopentanoic acid: $\mathrm{C}_{5} \mathrm{H}_{11} \mathrm{NO}_{2}$

${ }^{2}$ Creatine; IUPAC NAME: 2-[carbamimidoyl(methyl)amino] acetic acid; $\mathrm{C}_{4} \mathrm{H}_{9} \mathrm{~N}_{3} \mathrm{O}_{2}$

${ }^{3} \mathrm{~N}$-methyl-5-hydroxitriptamine, IUPAC NAME: 3-[2-(methylamino)ethyl]-1H-indol-5-ol

Highlighted rows indicate the studies which assessed TRP, KYN and KYNA simultaneously

3HAA 3-hydroxyanthranilic acid, 3HK 3-hydroxy-kynurenine, 5HIAA 5-hydroxy-indole-acetic acid, 5-HT 5-hydroxitriptamine, AA anthranilic acid, $C S F$ cerebrospinal fluid, FAA N-formylanthranilic acid, FLD fluorescent detector, $I S$ internal standard, KYN kynurenine, KYNA kynurenic acid, $M e-T R P$ methyl-tryptophan, $M e-5 H T$ N-methyl-5-hydroxytryptamine, $N A$ nicotinic acid, $N E O$ neopterin, $N F K$ N-formyl-kynurenine, $P A$ picolinic acid, $Q A$ quinolinic acid, TRP tryptophan, $U V D U V-V$ is detector, $X A$ xanthurenic acid 


\section{References for Table S1.}

[1] E.R. Werner, G. Bitterlich, D. Fuchs, A. Hausen, G. Reibnegger, G. Szabo, M.P. Dierich, H. Wachter, Human macrophages degrade tryptophan upon induction by interferon-gamma, Life Sci. 41 (1987) 273280.

[2] G. Werner-Felmayer, E.R. Werner, D. Fuchs, A. Hausen, G. Reibnegger, H. Wachter, Characteristics of interferon induced tryptophan metabolism in human cells in vitro, Biochim. Biophys. Acta Mol. Cell Res. 1012 (1989) 140-147.

[3] C. Hervé, P. Beyne, H. Jamault, E. Delacoux, Determination of tryptophan and its kynurenine pathway metabolites in human serum by high-performance liquid chromatography with simultaneous ultraviolet and fluorimetric detection, J. Chromatogr. B. Biomed. Sci. App. 675 (1996) 157-161.

[4] B. Widner, E.R. Werner, H. Schennach, H. Wachter, D. Fuchs, Simultaneous measurement of serum tryptophan and kynurenine by HPLC, Clin. Chem. 43 (1997) 2424-2426.

[5] H.Q. Wu, P. Guidetti, J.H. Goodman, M. Varasi, G. Ceresoli-Borroni, C. Speciale, H.E. Scharfman, R. Schwarcz, Kynurenergic manipulations influence excitatory synaptic function and excitotoxic vulnerability in the rat hippocampus in vivo, Neuroscience. 97 (2000) 243-251.

[6] C. Dazzi, G. Candiano, S. Massazza, A. Ponzetto, L. Varesio, New high-performance liquid chromatographic method for the detection of picolinic acid in biological fluids, J. Chromatogr. B. Biomed. Sci. App. 751 (2001) 61-68.

[7] D. Pawlak, A. Tankiewicz, W. Buczko, Kynurenine and its metabolites in the rat with experimental renal insufficiency, J. Physiol. Pharmacol. Off. J. Pol. Physiol. Soc. 52 (2001) 755-766.

[8] S. Fujigaki, K. Saito, M. Takemura, N. Maekawa, Y. Yamada, H. Wada, M. Seishima, 1-tryptophan-1kynurenine pathway metabolism accelerated by toxoplasma gondii infection is abolished in gamma interferon-gene-deficient mice: cross-regulation between inducible nitric oxide synthase and indoleamine-2,3-dioxygenase, Infection and Immunity. 70 (2002) 779-786.

[9] A. Laich, G. Neurauter, B. Widner, D. Fuchs, More rapid method for simultaneous measurement of tryptophan and kynurenine by HPLC, Clin. Chem. 48 (2002) 579-581.

[10] B. Widner, F. Leblhuber, D. Fuchs, Increased neopterin production and tryptophan degradation in advanced Parkinson's disease, J. Neural Transm. 109 (2002) 181-189.

[11] M. Pertovaara, A. Raitala, H. Uusitalo, J. Pukander, H. Helin, S.S. Oja, M. Hurme, Mechanisms dependent on tryptophan catabolism regulate immune responses in primary Sjögren's syndrome, Clin. Exp. Immunol. 142 (2005) 155-161. 
[12] J. Vignau, M.-C. Jacquemont, A. Lefort, M. Imbenotte, M. Lhermitte, Simultaneous determination of tryptophan and kynurenine in serum by HPLC with UV and fluorescence detection, Biomed. Chromatogr. 18 (2004) 872-874.

[13] N. Stoy, G.M. Mackay, C.M. Forrest, J. Christofides, M. Egerton, T.W. Stone, L.G. Darlington, Tryptophan metabolism and oxidative stress in patients with Huntington's disease, J. Neurochem. 93 (2005) 611-623.

[14] S.L. Hwang, N.P.-Y. Chung, J.K.-Y. Chan, C.-L.S. Lin, Indoleamine 2, 3-dioxygenase (IDO) is essential for dendritic cell activation and chemotactic responsiveness to chemokines, Cell Res. 15 (2005) 167175.

[15] G.M. Mackay, C.M. Forrest, N. Stoy, J. Christofides, M. Egerton, T.W. Stone, L.G. Darlington, Tryptophan metabolism and oxidative stress in patients with chronic brain injury, Eur. J. Neurol. 13 (2006) 30-42.

[16] K. Schröcksnadel, B. Wirleitner, C. Winkler, D. Fuchs, Monitoring tryptophan metabolism in chronic immune activation, Clin. Chim. Acta. 364 (2006) 82-90.

[17] C.M. Forrest, G.M. Mackay, N. Stoy, T.W. Stone, L.G. Darlington, Inflammatory status and kynurenine metabolism in rheumatoid arthritis treated with melatonin, Br. J. Clin. Pharmacol. 64 (2007) 517-526.

[18] G.J. Guillemin, K.M. Cullen, C.K. Lim, G.A. Smythe, B. Garner, V. Kapoor, O. Takikawa, B.J. Brew, Characterization of the kynurenine pathway in human neurons, J. Neurosci. 27 (2007) 12884-12892.

[19] A.-M. Myint, Y.K. Kim, R. Verkerk, S. Scharpé, H. Steinbusch, B. Leonard, Kynurenine pathway in major depression: evidence of impaired neuroprotection, J. Affect. Disord. 98 (2007) 143-151.

[20] A.A.B. Badawy, C.J. Morgan, Rapid isocratic liquid chromatographic separation and quantification of tryptophan and six kynurenine metabolites in biological samples with ultraviolet and fluorimetric detection, Int. J. Tryptophan Res. 3 (2010) 175-186.

[21] E. Gulaj, K. Pawlak, B. Bien, D. Pawlak, Kynurenine and its metabolites in Alzheimer's disease patients, Adv. Med. Sci. 55 (2010) 204-211.

[22] J. Zhao, P. Gao, D. Zhu, Optimization of Zn2+-containing mobile phase for simultaneous determination of kynurenine, kynurenic acid and tryptophan in human plasma by high performance liquid chromatography, J. Chromatogr. B Analyt. Technol. Biomed. Life. Sci. 878 (2010) 603-608.

[23] R.D. Oades, M.R. Dauvermann, B.G. Schimmelmann, M.J. Schwarz, A.M. Myint, Attention-deficit hyperactivity disorder (ADHD) and glial integrity: S100B, cytokines and kynurenine metabolism effects of medication, Behav. Brain Funct. 6 (2010) 29.

[24] L. Krcmova, D. Solichova, B. Melichar, M. Kasparova, J. Plisek, L. Sobotka, P. Solich, Determination of neopterin, kynurenine, tryptophan and creatinine in human serum by high throuput HPLC, Talanta. 85 (2011) 1466-1471. 
[25] H. Baran, J.A. Hainfellner, B. Kepplinger, Kynurenic acid metabolism in various types of brain pathology in HIV-1 infected patients, Int. J. Tryptophan Res. 5 (2012) 49-64.

[26] K.R. Linderholm, E. Skogh, S.K. Olsson, M.-L. Dahl, M. Holtze, G. Engberg, M. Samuelsson, S. Erhardt, Increased levels of kynurenine and kynurenic acid in the CSF of patients with schizophrenia, Schizophr. Bull. 38 (2012) 426-432.

[27] J. Zhao, H. Chen, P. Ni, B. Xu, X. Luo, Y. Zhan, P. Gao, D. Zhu, Simultaneous determination of urinary tryptophan, tryptophan-related metabolites and creatinine by high performance liquid chromatography with ultraviolet and fluorimetric detection, J. Chromatogr. B Analyt. Technol. Biomed. Life. Sci. 879 (2011) 2720-2725.

[28] J. Zhao, Plasma kynurenic acid/tryptophan ratio: a sensitive and reliable biomarker for the assessment of renal function, Ren. Fail. 35 (2013) 648-653.

[29] W.G. Lesniak, A. Jyoti, M.K. Mishra, N. Louissaint, R. Romero, D.C. Chugani, S. Kannan, R.M. Kannan, Concurrent quantification of tryptophan and its major metabolites, Anal. Biochem. 443 (2013) $222-231$.

[30] C.K. Lim, M.M.C. Yap, S.J. Kent, G. Gras, B. Samah, J.C. Batten, R. De Rose, B. Heng, B.J. Brew, G.J. Guillemin, Characterization of the kynurenine pathway and quinolinic acid production in macaque macrophages, Int. J. Tryptophan Res. 6 (2013) 7-19.

[31] M.J. Schwarz, G.J. Guillemin, S.J. Teipel, K. Buerger, H. Hampel, Increased 3-Hydroxykynurenine serum concentrations differentiate Alzheimer's disease patients from controls, Eur. Arch. Psychiatry Clin. Neurosci. 263 (2013) 345-352.

[32] S.M. Gibney, B. McGuinness, C. Prendergast, A. Harkin, T.J. Connor, Poly I:C-induced activation of the immune response is accompanied by depression and anxiety-like behaviours, kynurenine pathway activation and reduced BDNF expression, Brain, Behavior, and Immunity. 28 (2013)

[33] L.K. Krcmova, B. Cervinkova, D. Solichova, L. Sobotka, L. Hansmanova, B. Melichar, P. Solich, Fast and sensitive HPLC method for the determination of neopterin, kynurenine and tryptophan in amniotic fluid, malignant effusions and wound exudates, Bioanalysis. 7 (2015) 2751-2762.

[34] Y. Wang, J. Zhao, L. Tan, Y. Huang, D. Li, S. Quan, M. Li, J. Pan, Bone marrow mesenchymal stem cells alleviate extracellular kynurenine levels, as detected by high-performance liquid chromatography, Inflammation. 38 (2015) 1450-1457.

[35] D. Sheipouri, R. Grant, S. Bustamante, D. Lovejoy, G.J. Guillemin, N. Braidy, Characterisation of the kynurenine pathway in skin-derived fibroblasts and keratinocytes, J. Cell. Biochem. 116 (2015) 903922. 
[36] S. Guloksuz, B. Arts, S. Walter, M. Drukker, L. Rodriguez, A.-M. Myint, M.J. Schwarz, R. Ponds, J. van Os, G. Kenis, B.P.F. Rutten, The impact of electroconvulsive therapy on the tryptophan-kynurenine metabolic pathway, Brain. Behav. Immun. 48 (2015) 48-52.

[37] G. Veres, M. Molnár, D. Zádori, M. Szentirmai, L. Szalárdy, R. Török, E. Fazekas, I. Ilisz, L. Vécsei, P. Klivényi, Central nervous system-specific alterations in the tryptophan metabolism in the 3nitropropionic acid model of Huntington's disease, Pharmacol. Biochem. Behav. 132 (2015) 115-124.

[38] S. Eminel, N. Jin, M. Rostami, S. Dibbert, U. Mrowietz, I. Suhrkamp, Dimethyl- and monomethylfumarate regulate indoleamine 2,3-dioxygenase (IDO) activity in human immune cells, Exp. Dermatol. 26 (2017) 685-690.

[39] Bie Josien, Guest Jade, Guillemin Gilles J., Grant Ross, Central kynurenine pathway shift with age in women, J. Neurochem. 136 (2016) 995-1003.

[40] S. Comai, A. Bertazzo, J. Vachon, M. Daigle, J. Toupin, G. Côté, G. Turecki, G. Gobbi, Tryptophan via serotonin/kynurenine pathways abnormalities in a large cohort of aggressive inmates: markers for aggression, Prog. Neuro-Psychopharmacol. Biol. Psychiatry. 70 (2016) 8-16.

[41] M.R. Keegan, S. Chittiprol, S.L. Letendre, A. Winston, D. Fuchs, A. Boasso, J. Iudicello, R.J. Ellis, Tryptophan metabolism and its relationship with depression and cognitive impairment among HIVinfected individuals, Int. J. Tryptophan Res. 9 (2016) 79-88.

[42] A. Sekine, Y. Kuroki, T. Urata, N. Mori, T. Fukuwatari, Inhibition of Large Neutral Amino Acid Transporters Suppresses Kynurenic Acid Production Via Inhibition of Kynurenine Uptake in Rodent Brain, Neurochem. Res. 41 (2016) 2256-2266.

[43] F.F. Jusof, S.M. Bakmiwewa, S. Weiser, L.K. Too, R. Metz, G.C. Prendergast, S.T. Fraser, N.H. Hunt, H.J. Ball, Investigation of the tissue distribution and physiological roles of indoleamine 2,3dioxygenase-2, Int. J. Tryptophan Res. 10 (2017) 1-12.

[44] H. Kubo, M. Hoshi, A. Mouri, C. Tashita, Y. Yamamoto, T. Nabeshima, K. Saito, Absence of kynurenine 3-monooxygenase reduces mortality of acute viral myocarditis in mice, Immunol. Lett. 181 (2017) 94100.

[45] M.F. Dario, T.B. Freire, C.A.S. de O. Pinto, M.S.A. Prado, A.R. Baby, M.V.R. Velasco, Tryptophan and kynurenine determination in human hair by liquid chromatography, J. Chromatogr. B Analyt. Technol. Biomed. Life Sci. 1065-1066 (2017) 59-62.

[46] K. O'Farrell, E. Fagan, T.J. Connor, A. Harkin, Inhibition of the kynurenine pathway protects against reactive microglial-associated reductions in the complexity of primary cortical neurons, Eur. J. Pharmacol. 810 (2017) 163-173.

[47] A. Oliveros, K. Wininger, J. Sens, M.K. Larsson, X.C. Liu, S. Choi, A. Faka, L. Schwieler, G. Engberg, S. Erhardt, D.S. Choi, LPS-induced cortical kynurenic acid and neurogranin-NFAT signaling is 
associated with deficits in stimulus processing during Pavlovian conditioning, J. Neuroimmunol. 313 (2017) 1-9.

[48] J. Bartosiewicz, T. Kaminski, K. Pawlak, M. Karbowska, A. Tankiewicz-Kwedlo, D. Pawlak, The activation of the kynurenine pathway in a rat model with renovascular hypertension, Exp. Biol. Med. 242 (2017) 750-761.

[49] M. Tufvesson-Alm, L. Schwieler, R. Schwarcz, M. Goiny, S. Erhardt, G. Engberg, Importance of kynurenine 3-monooxygenase for spontaneous firing and pharmacological responses of midbrain dopamine neurons: Relevance for schizophrenia, Neuropharmacology. 138 (2018) 130-139.

[50] N. Michels, G. Clarke, L. Olavarria-Ramirez, S. Gómez-Martínez, L.E. Díaz, A. Marcos, K. Widhalm, L.A. Carvalho, Psychosocial stress and inflammation driving tryptophan breakdown in children and adolescents: A cross-sectional analysis of two cohorts, Psychoneuroendocrinology. 94 (2018) 104-111.

[51] M. Bochniarz, T. Kocki, R. Dąbrowski, M. Szczubiał, W. Wawron, W.A. Turski, Tryptophan, kynurenine, kynurenic acid concentrations and indoleamine 2,3-dioxygenase activity in serum and milk of dairy cows with subclinical mastitis caused by coagulase-negative staphylococci, Reprod. Domest. Anim. (2018).

[52] L. O’Rourke, G. Clarke, A. Nolan, C. Watkins, T.G. Dinan, C. Stanton, R.P. Ross, C.A. Ryan, Tryptophan metabolic profile in term and preterm breast milk: implications for health, J. Nutr. Sci. 7 (2018) e13.

[53] P. Giménez-Gómez, M. Pérez-Hernández, M.D. Gutiérrez-López, R. Vidal, C. Abuin-Martínez, E. O'Shea, M.I. Colado, Increasing kynurenine brain levels reduces ethanol consumption in mice by inhibiting dopamine release in nucleus accumbens, Neuropharmacology. 135 (2018) 581-591. 


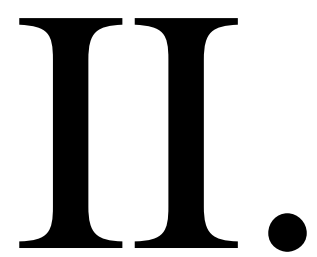




\title{
Neurotransmitter and tryptophan metabolite concentration changes in the complete Freund's adjuvant model of orofacial pain
}

Edina K. Cseh ${ }^{1 \dagger}$, Gábor Veres ${ }^{1,2+}$, Tamás Körtési ${ }^{1}$, Helga Polyák ${ }^{1}$, Nikolett Nánási ${ }^{1}$, János Tajti ${ }^{1}$, Árpád Párdutz ${ }^{1}$, Péter Klivényi ${ }^{1}$, László Vécsei ${ }^{1,2}$ and Dénes Zádori ${ }^{{ }^{*}}$

\begin{abstract}
Background: The neurochemical background of the evolution of headache disorders, still remains partially undiscovered. Accordingly, our aim was to further explore the neurochemical profile of Complete Freund's adjuvant (CFA)-induced orofacial pain, involving finding the shift point regarding small molecule neurotransmitter concentrations changes vs. that of the previously characterized headache-related neuropeptides. The investigated neurotransmitters consisted of glutamate, $\gamma$-aminobutyric acid, noradrenalin and serotonin. Furthermore, in light of its influence on glutamatergic neurotransmission, we measured the level of kynurenic acid (KYNA) and its precursors in the kynurenine (KYN) pathway (KP) of tryptophan metabolism.

Methods: The effect of CFA was evaluated in male Sprague Dawley rats. Animals were injected with CFA ( $1 \mathrm{mg} / \mathrm{ml}$, $50 \mu \mathrm{l} / \mathrm{animal}$ ) into the right whisker pad. We applied high-performance liquid chromatography to determine the concentrations of the above-mentioned compounds from the trigeminal nucleus caudalis (TNC) and somatosensory cortex ( $s \mathrm{SCX}$ ) of rats. Furthermore, we measured some of these metabolites from the cerebrospinal fluid and plasma as well. Afterwards, we carried out permutation t-tests as post hoc analysis for pairwise comparison.

Results: Our results demonstrated that $24 \mathrm{~h}$ after CFA treatment, the level of glutamate, KYNA and that of its precursor, KYN was still elevated in the TNC, all diminishing by $48 \mathrm{~h}$. In the $\mathrm{ssCX}$, significant concentration increases of KYNA and serotonin were found.

Conclusion: This is the first study assessing neurotransmitter changes in the TNC and ssCX following CFA treatment, confirming the dominant role of glutamate in early pain processing and a compensatory elevation of KYNA with anti-glutamatergic properties. Furthermore, the current findings draw attention to the limited time interval where medications can target the glutamatergic pathways.
\end{abstract}

Keywords: Migraine, CFA model, Orofacial pain, Glutamate, Kynurenic acid

\footnotetext{
* Correspondence: zadori.denes@med.u-szeged.hu

†Edina K. Cseh and Gábor Veres contributed equally to this work.

'Department of Neurology, Interdisciplinary Excellence Center, Faculty of

Medicine, Albert Szent-Györgyi Clinical Center, University of Szeged,

Semmelweis u. 6, Szeged H-6725, Hungary

Full list of author information is available at the end of the article
}

(c) The Author(s). 2020 Open Access This article is licensed under a Creative Commons Attribution 4.0 International License, which permits use, sharing, adaptation, distribution and reproduction in any medium or format, as long as you give appropriate credit to the original author(s) and the source, provide a link to the Creative Commons licence, and indicate if changes were made. The images or other third party material in this article are included in the article's Creative Commons licence, unless indicated otherwise in a credit line to the material. If material is not included in the article's Creative Commons licence and your intended use is not permitted by statutory regulation or exceeds the permitted use, you will need to obtain permission directly from the copyright holder. To view a copy of this licence, visit http://creativecommons.org/licenses/by/4.0/ The Creative Commons Public Domain Dedication waiver (http://creativecommons.org/publicdomain/zero/1.0/) applies to the data made available in this article, unless otherwise stated in a credit line to the data. 


\section{Background}

Although the pathomechanism of orofacial pain and headache disorders, is not fully understood [1], the activation and sensitization of the trigeminovascular system (TS) probably takes part in the evolution of symptoms [2-4]. The pathomechanism of these disorders may be further investigated by using animal models with the activation of nociceptive pathways of the TS $[1,3,5]$. The administration of inflammation-inducing substances to the orofacial area can evoke the above-described activation/sensitization of the primary and secondary trigeminal neurons during pain processing [6, 7]. For the induction of this peripheral inflammation, the application of Complete Freund's adjuvant (CFA) into the whisker pad or the dural parietal surface is a widely used method $[6,8,9]$ as it is able to enhance local reaction at the injection site and then to evoke the release of inflammatory cytokines, alongside with hyperalgesia/allodynia on the face via the activation/sensitization of the TS [7]. Regarding the delay of the development of peripheral and central sensitization, indirect data from studies with CFA injection to the paw demonstrated that pain hypersensitivities were observed $24 \mathrm{~h}$ after the injection [9-13], whereas data from studies with orofacial CFA model, more precisely from the temporomandibular joint induced inflammation model, suggest that both thermal and mechanical allodynia peak at $24 \mathrm{~h}$ as well [14]. The orofacial CFA model has been thoroughly studied regarding gene expression characteristics $[6,15-$ $20]$. Recently, in relation to two migraine-related biomarkers, the pituitary adenylate cyclase-activating peptide (PACAP) and calcitonin gene-related peptide (CGRP), their increasing levels were detected starting even $24 \mathrm{~h}$ after the administration of CFA in the trigeminal nucleus caudalis (TNC) [9]. However, there are no studies which aimed at the investigation of the small molecule neurotransmitters and neuromodulators and some of their precursors (glutamate (Glu), $\gamma$ aminobutyric acid (GABA), setotonin (5-hydroxy-tryptamine; 5-HT), noradrenaline (NA), tryptophan (TRP), kynurenine (KYN), kynurenic acid (KYNA)) in this model with established or presumed role in the development of peripheral and central sensitization during headache. Therefore, there are no data about how the concentration changes of these substances affect the evolution of peripheral and central sensitization. Accordingly, finding the transition point where the dominance of small molecule mediated neurotransmission shifts to that of the PACAP and CGRP mentioned earlier may have significant therapeutic consequences in view of the different targeted approaches.

The primary excitatory neurotransmitter Glu plays an important role in the primary sensory neurotransmission and trigeminal nociception $[15,21,22]$.
Accordingly, the alteration of Glu levels in migraine has been widely studied and data consistently show elevated Glu levels in the CSF samples of patients with chronic migraine [23], or migraine with and without aura [24], whereas in plasma samples, the results were not consistent across studies [25-27]. Moreover, similar importance has to be attributed to the changes of the concentration of GABA, the main inhibitory neurotransmitter of the central nervous system (CNS), which is capable of modulating the excitatory pathways [28]. Recently, mainly in light of its influence on glutamatergic neurotransmission, special attention was dedicated to the investigation of the effect of KYNA, a compound of the KYN pathway (KP) of the TRP metabolism [29-34]. KYNA can influence glutamatergic neurotransmission in a complex way [35], i.e., it acts as a competitive antagonist at the Nmethyl-D-aspartate (NMDA) receptor [36] and has weak antagonistic effects at the $\alpha$-amino-3-hydroxy-5methyl-4-isoxazolepropionic acid (AMPA) and kainate receptors as well [37]. 5-HT, another well-known TRP metabolite, released from serotonergic neurons of the raphe nuclei, exerts modulating effect on TS activation [38-40]. Noradrenaline (NA) may be of interest as well, as noradrenergic neurons project to TNC and may have a role in cluster headache, another primary headache disorder [41, 42].

Based on the observed gradually increasing levels of PACAP and CGRP from $24 \mathrm{~h}$ following CFA injection in our previous experiment [9], the aim of the current study was to find the shift point of concentration changes of small molecule neurotransmitters and neuromodulators and the above-mentioned peptides. This may yield substantial information for the selection between different therapeutic paradigms regarding diseases involving the activation of the TS, such as primary headache disorders, including migraine.

\section{Materials and methods \\ Animal experiments and sample collection}

Twenty-seven young adult (10-12 weeks old, 250-300 g) male Sprague-Dawley rats (Charles River Laboratories, Wilmington, MA, USA), were used for the experiments. The animals were bred and maintained under standard laboratory conditions with $12 \mathrm{~h}-12 \mathrm{~h}$ light/dark cycle at $24 \pm 1{ }^{\circ} \mathrm{C}$ and $50 \%$ relative humidity, 3 animals per each home cage in the Laboratory Animal House of the Department of Neurology, University of Szeged. The rats had free access to standard rat chow and water. The experiment was not pre-registered. All experimental procedures performed in this study complied fully with the guidelines of Act 1998/XXVIII of the Hungarian Parliament on Animal Experiments (243/1988) and with the recommendations of the International Association for 
the Study of Pain and European Communities Council (86/609/ECC). The studies were in harmony with the Ethical Codex of Animal Experiments and were approved by the Ethics Committee of the Faculty of Medicine, University of Szeged, with a permission number of XI./1102/2018. CFA (killed mycobacteria suspended in paraffin oil, $1 \mathrm{mg} / \mathrm{ml}$ ) was obtained from Sigma-Aldrich (product number: F5881; St. Louis, MO, USA), and $50 \mu \mathrm{l}$ was administered per animal. We tried to minimalize the use of animals by adopting the key aspects of the 3Rs (Replacement, Reduction and Refinement) [43]. Therefore, the experimental groups were added in a sequential manner, starting from $24 \mathrm{~h}$ following CFA administration with $24 \mathrm{~h}$ steps till the time point where the proposed alterations diminish. Therefore, no randomization was performed to allocate subjects in the study. By the end of the experiments we had three groups, one control $(\mathrm{CO})$ and two with CFA treatment (Fig. 1). Similar to the previous experiment on PACAP and CGRP in the same model [9], only sham-injected rats processed $24 \mathrm{~h}$ following the injection were used as $\mathrm{CO}$, as a pilot study conducted on naive and shaminjected (processed 24 and $48 \mathrm{~h}$ following injection) rats demonstrated that there is no difference in the level of the metabolites of interest, in neither TNC, nor ssCX ( $n=3$ in each group, data not shown). The rats were anesthetized with intraperitoneal $4 \%$ chloral hydrate solution mainly based on its safe application (CAS ID: 302-17-0, Sigma-Aldrich, St. Louis, MO, USA; $10 \mathrm{ml} / \mathrm{kg}$ body weight dose) in the morning and $50 \mu$ lof CFA was injected into the right whisker pad. No other analgesic was applied, otherwise the activation/sensitization phenomena during pain processing, an essential characteristic of the CFA model as well, would have been influenced. Control rats were injected with an equal volume of saline. Cerebrospinal fluid (CSF) was taken from the suboccipital cistern, including the control group $(n=9), 24(n=9)$ and $48 \mathrm{~h}(\mathrm{n}=9$ initially, finally $n=8$ as one animal died during the experiment) after injection applying the above-described anesthetic procedure, and following that the animals were perfused transcardially with $200 \mathrm{ml}$ phosphate-buffered saline (PBS). The spinal tap procedures were unsuccessful in 5 occasions and 7 of the CSF samples were excluded from analysis due to contamination with blood. Accordingly, 5-5 samples remained in the $\mathrm{CO}$ and CFA $24 \mathrm{~h}$ groups, and 4 in the CFA $48 \mathrm{~h}$ group for analysis. Therefore, this part of the study focusing at that secondary endpoint was only exploratory due to the low statistical power. Also as a secondary endpoint, blood samples were taken from the left ventricle into ice-cold glass tubes containing disodium ethylenediaminetetraacetate dihydrate $\left(\mathrm{Na}_{2} \mathrm{EDTA}\right.$; CAS ID: 194491-31-1 Lach-Ner s.r.o, Neratovice, Czech Republic) and the plasma was separated by centrifugation (1170 g for $10 \mathrm{~min}$ at $4^{\circ} \mathrm{C}$ ). Following decapitation two different brain structures, the TNC and the somatosensory cortex ( $\mathrm{ssCX}$ ) were dissected for the assessment of the targeted primary endpoints. In each case both right and left sided samples were separately removed on ice and stored at $-80^{\circ} \mathrm{C}$ until further use.

\section{Instruments and chromatographic conditions}

Validated high performance liquid chromatography (HPLC) measurements were performed by an Agilent 1100 HPLC system (Santa Clara, CA, USA), coupled with UV detector (UVD), fluorescence detector (FLD) and electrochemical detector (ECD). The chromatographic separations were carried out with validated methods comprehensively described elsewhere [44-46]. Prior to all measurements, during the tissue weighting or plasma/CSF precipitation process, all samples were relabeled, and a blind study was conducted, i.e., the experimenter who did the HPLC measurements was not aware of which samples were part of $\mathrm{CO}$ or $24 \mathrm{~h}$ groups. Moreover, Eppendorf tubes were randomly assigned for measurements and when the $48 \mathrm{~h}$ group was measured, the same systematic randomization was applied. The purity of all standards and solutions were analytical grade or HPLC grade and they were acquired from Sigma-Aldrich, St. Louis, MO, USA, except the

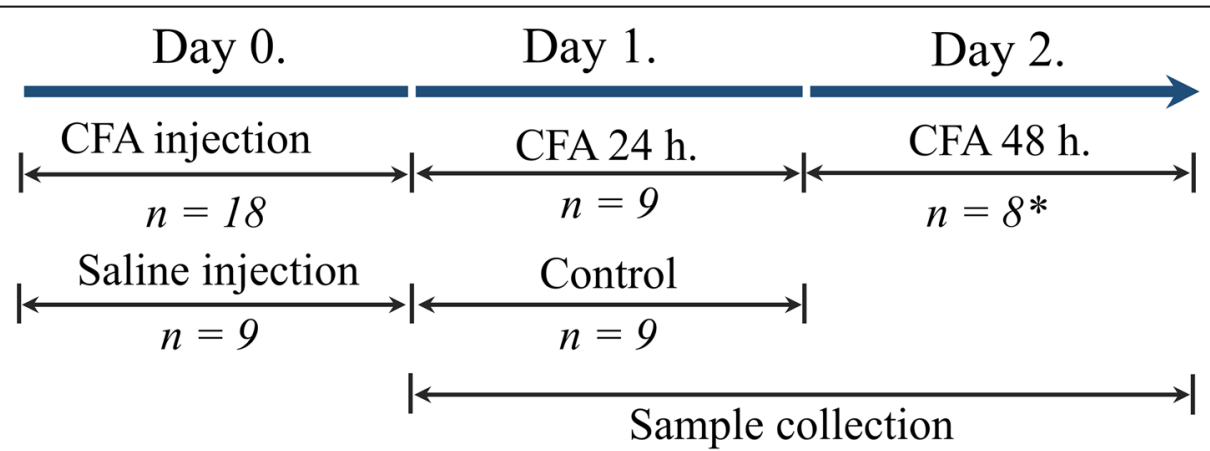

Fig. 1 Time-line of the experimental procedure applied in this study. CFA Complete Freund's adjuvant. $n$ number of the animals per group. ${ }^{*}$ One animal died in cage after CFA injection 
fluorescent internal standard used for the TRP method which was synthesized at the Department of Pharmaceutical Chemistry, University of Szeged, as detailed elsewhere [44]. Briefly, the brain regions were homogenized in $0.5 \mathrm{M}$ perchloric acid (PCA), at $1: 5 \mathrm{w} / \mathrm{v}$ containing internal standards (ISs, 3-nitro-L-tyrosine and 4-hydroxyquinazoline-2-carboxylic acid, the latter custom-made material will be shared upon reasonable request) applied in the measurement of TRP metabolites [44] utilizing both UVD and FLD. After centrifugation the supernatant was collected and first used for the TRP metabolite measurement. The remaining supernatant was aliquoted in two further parts and were kept at $80^{\circ} \mathrm{C}$ until further analyses. $150 \mu \mathrm{l}$ from it was applied for the determination of NA concentration by ECD [45], with addition of $10 \mu \mathrm{l}$ solution of the corresponding IS, 2,3-dihydroxybenzoic acid. For the measurement of Glu and GABA, another $100 \mu \mathrm{l}$ was diluted to $1: 100 \mathrm{v} / \mathrm{v}$ with distilled water and $100 \mu \mathrm{l}$ of this dilution was derivatized with $100 \mu \mathrm{l}$ solution containing o-phthaldialdehyde and 3 -mercaptopropionic acid in borate buffer and further diluted with $50 \mu \mathrm{l}$ distilled water containing the corresponding IS, homoserine, used for this method applying FLD [46].

For the measurement of the TRP metabolites from the CSF, the method described before [44] was applied, with a slight modification. Briefly, during sample preparation, we used a dilution of $5: 6 \mathrm{v} / \mathrm{v}$, with the final concentration of PCA at $0.5 \mathrm{M}$, with the above described ISs, but only $35 \mu \mathrm{L}$ of the sample was injected. Furthermore, a linearity study was conducted for rat CSF samples to determine limit of detection (LOD) and limit of quantitation (LOQ) values, because the cited article contains data only for human CSF. Accordingly, the LOD and LOQ values for rat CSF were 31.1 and $102 \mathrm{nM}$ for TRP, 107 and $702 \mathrm{nM}$ for $\mathrm{KYN}$ and 1.04 and $3.45 \mathrm{nM}$ for KYNA, respectively, whereas 5-HT was undetectable in each case. Regarding Glu and GABA, the initial amount of mobile phase A applied for the brain samples was $95 \%$, but for CSF samples it was changed to $93 \%$, as coelution was observed under the initial circumstances. The ratios applied for the CSF sample preparation (1:1:0.5= sample: derivatization solution: IS) remained the same, similar to brain supernatants [46]. Due to low sample amount we omitted the determination of NA levels from CSF.

With regard to plasma samples we measured the levels of TRP metabolites as described in [44]. Glu, GABA and NA concentrations from plasma samples were not assessed because we were only interested in their role as a neurotransmitter.

As for the plasma samples, the LOD and LOQ values were $0.102 \mu \mathrm{M}$ and $0.308 \mu \mathrm{M}$ for TRP, 0.027 and $0.083 \mu \mathrm{M}$ for $\mathrm{KYN}$ and 1.23 and $3.72 \mathrm{nM}$ for KYNA, respectively. In each case, the 5-HT levels from plasma samples were undetectable.

\section{Statistical analyses}

All statistical calculations were performed with the use of the freely available R software 3.5.3 (R Development

Table 1 Concentration levels of the measured metabolites in the analyzed brain regions

\begin{tabular}{|c|c|c|c|}
\hline & Control group $(n=9)$ & CFA $24 \mathrm{~h}(n=9)$ & CFA $48 \mathrm{~h}\left(n=8^{\dagger}\right)$ \\
\hline \multicolumn{4}{|c|}{ Trigeminal nucleus caudalis (TNC) } \\
\hline Glu ( $\mu g / g w w)$ & $684(644-746)$ & $772^{*}, \#(742-859)$ & $731(687-745)$ \\
\hline GABA ( $\mu g / g w w)$ & $167(154-187)$ & $180(174-235)$ & $167(164-171)$ \\
\hline TRP (nmol/g ww) & $20.3(19.2-22.4)$ & $20.3(18.2-24.5)$ & $19.4(17.7-20.8)$ \\
\hline $\mathrm{KYN}(\mathrm{nmol} / \mathrm{g} w w)$ & $0.656(0.428-0.671)$ & $0.876^{*}, \#(0.830-1.13)$ & $0.532(0.480-0.597)$ \\
\hline KYNA (pmol/g ww) & $22.8(21.2-24.2)$ & $52.6^{* * \# \#}(34.6-72.3)$ & $25.8(21.9-28.8)$ \\
\hline 5-HT (pmol/g ww) & 2991 (2917-3333) & $2841(2629-3425)$ & 3315 (3088-3438) \\
\hline$N A(\mu g / g w w)$ & $0.328(0.320-0.343)$ & $0.352(0.328-0.388)$ & $0.348(0.324-0.366)$ \\
\hline \multicolumn{4}{|c|}{ Somatosensory cortex (ssCX) } \\
\hline Glu ( $\mu \mathrm{g} / \mathrm{g} w w)$ & $1178(1082-1290)$ & 1269 (1206-1397) & $1152(1052-1287)$ \\
\hline GABA ( $\mu g / g w w)$ & $215(207-218)$ & $230(217-251)$ & $199(178-211)$ \\
\hline TRP (nmol/g ww) & $20.6(17.8-23.5)$ & $22.6(21.5-23.7)$ & $21.6(20.9-22.7)$ \\
\hline $\mathrm{KYN}(\mathrm{nmol} / \mathrm{g} w w)$ & $0.824(0.743-0.970)$ & $0.974(0.714-1.15)$ & $0.616(0.552-0.663)$ \\
\hline KYNA (pmol/g ww) & $16.2(9.70-18.8)$ & $27.3^{*}, \#(17.3-39.3)$ & $9.73(7.01-12.8)$ \\
\hline 5-HT (pmol/g ww) & $2547(1665-2677)$ & $2271^{\#}(2166-2527)$ & $2885(2653-3172)$ \\
\hline$N A(\mu g / g w w)$ & $0.840(0.192-0.853)$ & $0.754(0.142-0.934)$ & $0.886(0.556-0.974)$ \\
\hline
\end{tabular}

Results are shown as median (1st-3rd quartile). ${ }^{\dagger}$ One animal died in cage after CFA injection. ${ }^{*} p<0.05$ vs. CO, ${ }^{* *} p<0.01$ vs. CO, \# $p<0.05$ vs. $48 \mathrm{~h}, 5-H T$ serotonin, CFA Complete Freund's adjuvant, GABA gamma-aminobutyric acid, Glu glutamate, $K Y N$ kynurenine, KYNA kynurenic acid, $n$ number of the animals per group, NA noradrenaline, TRP tryptophan, wW wet weight 

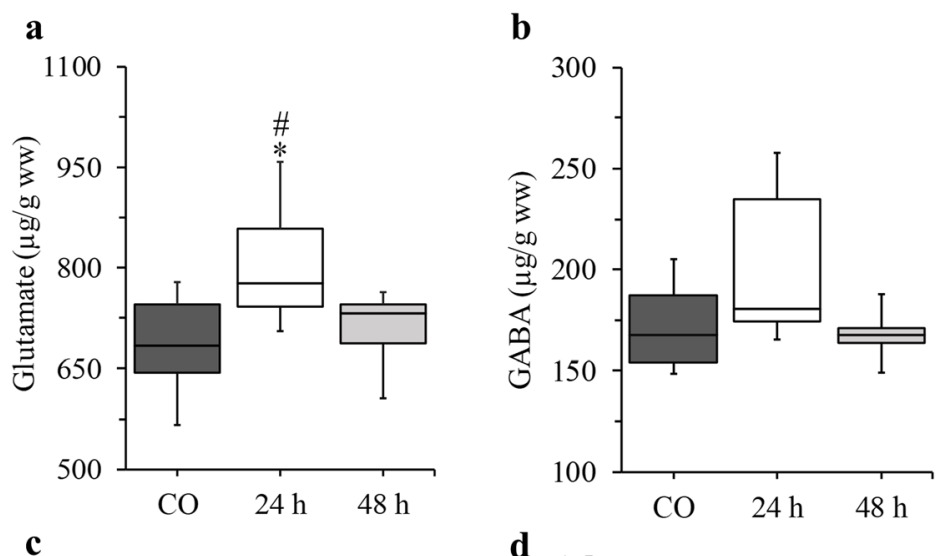

c

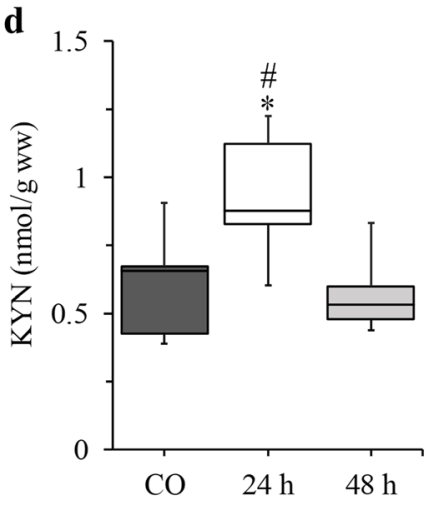

e

f
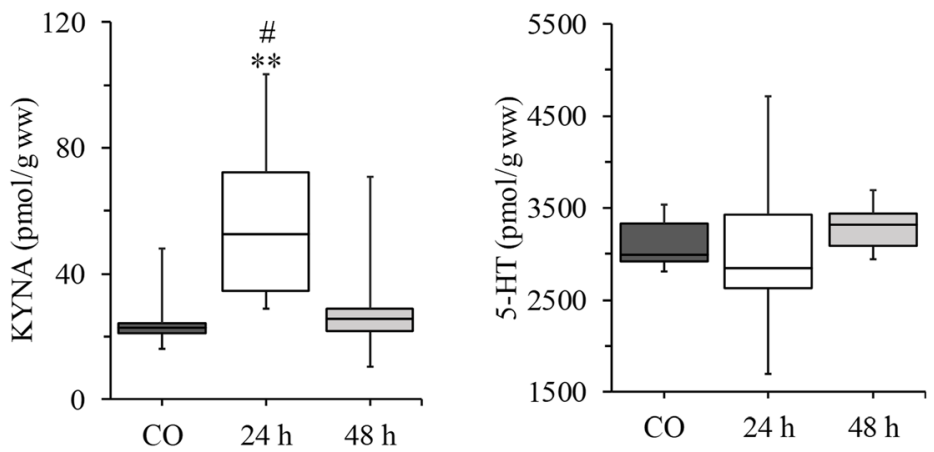

g

h
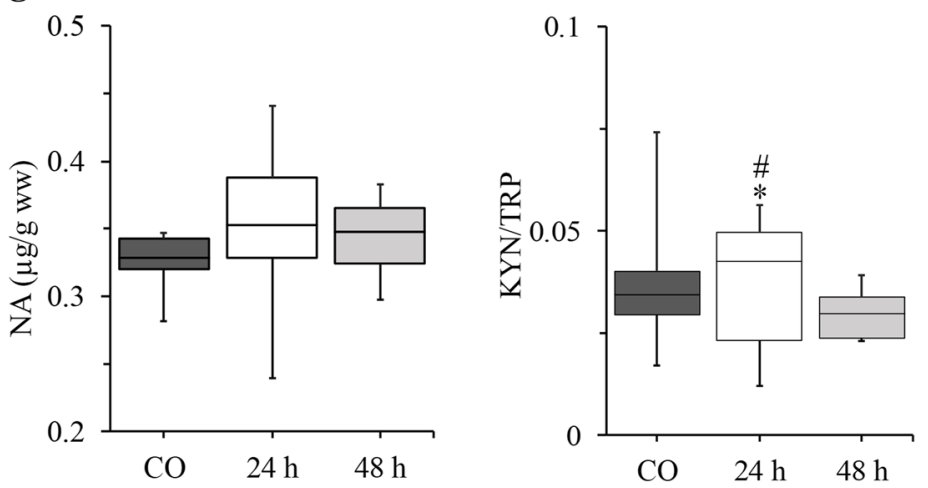

Fig. 2 (See legend on next page.) 
(See figure on previous page.)

Fig. 2 Concentration changes in glutamate $(\mathbf{a}), y$-aminobutyric acid (b), tryptophan (c), kynurenine $(\mathbf{d})$, kynurenic acid (e), serotonin (f), noradrenaline $(\mathbf{g})$ and changes in kynurenine/tryptophan ratio (h) in the TNC. ${ }^{*} p<0.05$ vs. CO, ${ }^{* *} p<0.01$ vs. CO, \# $p<0.05$ vs. 48 h. $n=9$ in the control and $24 \mathrm{~h}$ groups and $n=8$ in the $48 \mathrm{~h}$ group. The boxplots are displayed as the intervals between the 1 st and 3 rd quartiles presenting the median values as well. 24 and 48 h CFA treated groups, 5-HT serotonin, CO control, GABA $y$-aminobutyric acid, KYN kynurenine, KYNA kynurenic acid, $n$ number of the animals per group, NA noradrenaline, TRP tryptophan, TNC trigeminal nucleus caudalis, wW wet weight

Core Team). The distribution of our data population was not determined as the applied statistical tests do not need assumptions regarding the distribution of underlying data. Accordingly, first we performed the Levene test to assess the homogeneity of variances. As the variances were equal, we performed a general independence test for two sets of variables measured on arbitrary scales, where the reference distribution was approximative based on the Monte-Carlo method. Afterwards, we carried out permutation t-tests as post hoc analysis for pairwise comparison. Permutations were applied via the Monte-Carlo method (10,000 random permutations) and Type I errors from multiple comparisons were controlled with false discovery rate. No test for outliers was conducted. With the key aspects of 3 Rs in mind [43] we tried to keep the sample size as low as we can based on experiences from previous experiments ([47]: 8 and 12/ group; [48]: 6/group; [49]: 6/group; [50]: 6/group; [51]: 8/group; [52]: 6 and 7/group; [53]: 6/group). For every statistically significant result, we calculated the corresponding effect size (Cohen's $d$ in this case) and based on its value, we decided whether the increase of sample size is necessary or not. The manuscript contains the final effect sizes.

\section{Results}

\section{Concentration levels of the assessed compounds in the TNC and ssCX}

First of all, both contralateral and ipsilateral CNS regions were measured separately, but we did not find significant differences in concentrations of any of the metabolites between the two sides, so the coherent data were pooled for further analysis. Therefore, the concentration values presented in Table 1 demonstrate the mean values of the two analyzed sides of each CNS regions.

Regarding TNC, pairwise permutation t-tests following the independence tests revealed a significant elevation in the concentration of Glu ( $p=0.0319$, Cohen's $\mathrm{d}=1.49)$, KYN $(p=0.0123$, Cohen's $\mathrm{d}=1.58)$ and KYNA $(p=$ 0.0098, Cohen's $\mathrm{d}=1.92) 24 \mathrm{~h}$ following CFA injection compared to the controls and a significant decrease could be observed in Glu ( $p=0.0357$, Cohen's $d=1.29)$, KYN $(p=0.0123$, Cohen's $\mathrm{d}=1.85)$ and KYNA $(p=$ 0.0263 , Cohen's $d=1.39$ ) levels by $48 \mathrm{~h}$ compared to the $24 \mathrm{~h}$ group, whereas there was no difference between the control and $48 \mathrm{~h}$ groups (Table 1, Fig. 2).
Regarding ssCX samples, an elevation in KYNA concentration $(p=0.0237$, Cohen's $\mathrm{d}=1.36)$ could be observed $24 \mathrm{~h}$ following CFA administration, followed by a significant decrease by $48 \mathrm{~h}(p=0.0173$, Cohen's $\mathrm{d}=$ $1.80)$ and there was no difference between control and $48 \mathrm{~h}$ groups. Furthermore, in the ssCX, there was a significant increase in 5-HT levels in the $48 \mathrm{~h}$ group compared to the controls $(p=0.0479$, Cohen's $\mathrm{d}=1.21)$ and to the $24 \mathrm{~h}$ group ( $p=0.0479$, Cohen's $\mathrm{d}=1.20$; Table 1 , Fig. 3).

We calculated the KYN/TRP and KYNA/KYN ratios as well. The KYN/TRP ratio was significantly elevated in the $24 \mathrm{~h}$ group compared to the controls $(p=0.0419$, Cohen's $\mathrm{d}=1.19)$ or to the $48 \mathrm{~h}$ group $(p=0.0419$, Cohen's $\mathrm{d}=1.35$; Table 1, Fig. 2). With regard to the KYNA/KYN ratio, there was no difference in any of the investigated biological matrices (data no shown).

\section{CSF and plasma samples}

Regarding CSF samples, TRP metabolites, Glu and GABA were measured. We found no significant alterations in the CSF, however, the power of the statistical tests in this case is low due to low case number $(n=5,5$, 4 for control, $24 \mathrm{~h}$ and $48 \mathrm{~h}$ groups, respectively) and the concentration values of KYN in the control and CFA treated $48 \mathrm{~h}$ groups were below LOD $(0.107 \mu \mathrm{M})$, except one case from each group (for more details, see Additional file 1, Table S1; due to the low amount of 5-HT in the CSF samples, we could not quantify it, as the values were below $\mathrm{LOD}, \mathrm{LOD}=0.0274 \mu \mathrm{M}$ ). In case of plasma samples, only the TRP metabolites were measured, and no significant differences were observed (for more details, see Additional file 2, Table S2).

\section{Discussion}

Headache is one of the most common neurological disorders and it is one of the leading causes of healthrelated problems worldwide. In 2010, tension type headache and migraine were the second and third most prevalent conditions in the world, respectively, according to the Global Burden of Disease (GBD) study [54, 55]. Furthermore, the GBD study in 2015 established that headache is responsible [56] for more disability adjusted life years than all other neurological disorders in combination.

The treatment of primary headache disorders is challenging, requiring both acute and preventive therapeutic 

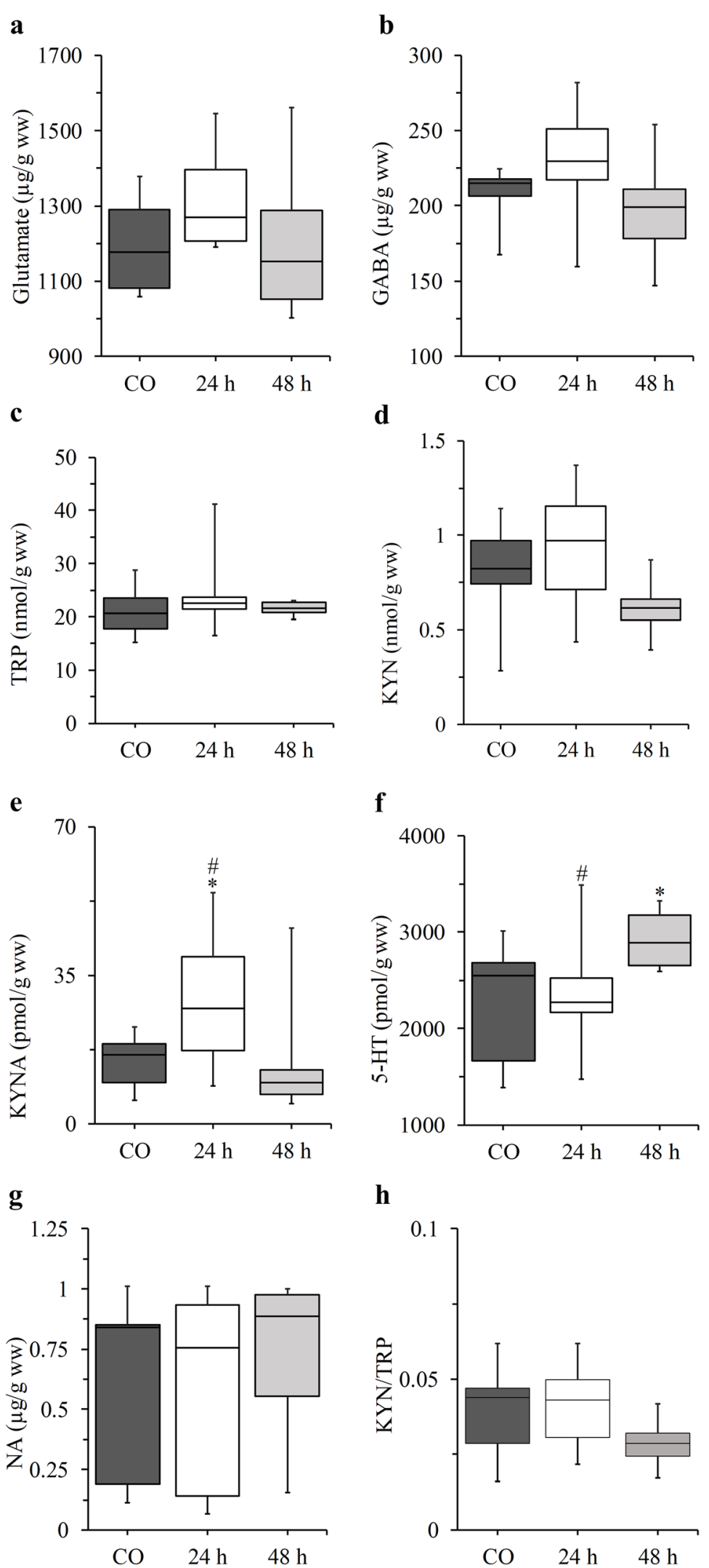

Fig. 3 (See legend on next page.) 
(See figure on previous page.)

Fig. 3 Concentration changes in glutamate $(\mathbf{a})$, $\gamma$-aminobutyric acid (b), tryptophan (c), kynurenine $(\mathbf{d})$, kynurenic acid (e), serotonin (f), noradrenaline $(\mathbf{g})$ and changes in kynurenine/tryptophan ratio $(\mathbf{h})$ in the somatosensory cortex. ${ }^{*} p<0.05$ vs. CO, $\# p<0.05$ vs. $48 \mathrm{~h}$. $\mathbf{n}=9$ in the control and $24 \mathrm{~h}$ groups and $n=8$ in the $48 \mathrm{~h}$ group. The boxplots are displayed as the intervals between the 1st and 3rd quartiles presenting the median values as well. 24 and 48 h CFA treated groups, 5-HT serotonin, CO control, GABA $\gamma$-aminobutyric acid, KYN kynurenine, KYNA kynurenic acid, $n$ number of the animals per group, NA noradrenaline, TRP tryptophan, TNC trigeminal nucleus caudalis, wW wet weight

measures $[57,58]$. The preventive treatment aims to reduce the frequency, severity and duration of headaches, and to avoid medication-overuse headache. The efficacy of the currently applied drugs is not always satisfactory and the contraindications and side-effects often limit the options of the physician $[59,60]$. Therefore, there is a constant need to study and develop new molecules.

\section{Glutamate and pain}

Peripheral and central sensitization manifest mainly in forms of hyperalgesia and allodynia. The activation of the peripheral terminals of the nociceptors is responsible for Glu release at central sites with the activation of ionotropic and metabotropic Glu receptors [61]. This process was demonstrated not only in preclinical studies [62-64], but in patients with headache as well [23, 24]. Accordingly, the role of glutamatergic pathways in association with different types of pain is well established [65] and several antagonists of ionotropic glutamate receptors were investigated and found to be effective to decrease nociceptive transmission [66]. However, they had severe side effects, and therefore, the interest in this direction of research diminished [67, 68]. Nevertheless, ketamine, an NMDA receptor antagonist, is so far the only promising option in the treatment of severe or long-lasting migraine aura [69], and tezampanel, which acts on the AMPA and kainate subtypes of ionotropic Glu receptors [70], has also shown promising results in acute migraine therapy [71].

\section{Tryptophan metabolism and pain}

It has been already demonstrated that the level of KYNA and some other KP metabolites are altered in migraine and cluster headache patients as well: there are significant reductions in the serum levels of KYN, KYNA, 3-hydroxy-kynurenine, 3-hydroxyanthranilic acid and quinolinic acid, whereas concentrations of TRP and anthranilic acid were significantly increased $[72,73]$. KYNA as an endogenous NMDA receptor antagonist, is a molecule of interest for CNS drug development in case of several neurological conditions [74], but due to its poor ability to cross the blood-brain barrier (BBB) and its rapid clearance from the body [75], its application for most CNS-related alterations is limited, and therefore several KYNA analogs were synthetized [76-79]. However, the first order neuron of pain processing is located outside the BBB [80], so KYNA itself may have therapeutic potential as well. Accordingly, the antinociceptive properties of KYNA were proved in animal models of pain [29, 81]. Furthermore, some of the developed analogs also displayed promising results in different animal models of headache [31, 82-85]. In an earlier study we investigated two KYNA analogs where both of them proved to be effective in the formalin model of trigeminal pain [84]. However, one of them was more effective than the other and according to our analyses the better performing compound caused a more pronounced elevation of KYNA concentration on the periphery, whereas in the CNS the concentrations of KYNA were similar. Based on these results we hypothesized that the peripheral elevation of KYNA may be enough to exert beneficial effects on pain processing and targeting this component could provide an option to pharmaceutical drug design without the obligation of good penetration through the BBB.

Elevated Glu concentration in the TNC of CFAtreated rats, demonstrated by the current study, is accompanied by increased KYN and KYNA levels, which may serve as a feedback mechanism to the sensitization process caused by Glu. This hypothesis is supported by the above-mentioned findings [72, 73] that decreased KP metabolite levels are associated with those headache disorders, where increased NMDA receptor activation may play a crucial role. These results may have a great importance especially in light of the finding that the slightly, but not significantly elevated GABA level may not be enough to counterbalance the effects of increased Glu levels. With regard to 5-HT, its cortical elevation by $48 \mathrm{~h}$ may serve as a feedback inhibitory response as well to ameliorate the activation of the trigeminovascular pathway [86].

The current study draws attention to the limited time interval for therapies targeting glutamatergic pathways as well, as based on our previous experiments, a clear shift to dominantly peptide-mediated pain processing can be seen even from $24 \mathrm{~h}$ after CFA application [9]. This time point corresponds to the onset of peripheral and central sensitization of the TS as well in this model [10, 11, 14]. At this stage, mainly novel antibody-based therapies may come into account [87-90]. With regard to these novel therapies, the focus of attention is on 
monoclonal antibodies targeting the CGRP pathway for the prophylactic treatment of migraine. Currently, four of these antibodies are in clinical trials (eptinezumab, galcanezumab, fremanezumab, erenumab) with promising results. However, the cost of these therapies is considerably higher than that of acute phase treatments.

\section{Conclusion}

This is the first study assessing small molecule neurotransmitter changes in the TNC and ssCX following CFA treatment, confirming a dominant role of glutamate in early pain processing and a compensatory elevation of KYNA with anti-glutamatergic properties. The time interval for the intervention targeting the glutamatergic system is presumed to be limited to the first $24 \mathrm{~h}$. The results of our previous therapeutic studies with KYNA or with its analogs strongly support this theory.

\section{Supplementary information}

Supplementary information accompanies this paper at https://doi.org/10. 1186/s10194-020-01105-6.

Additional file 1: Table S1. Concentration levels of the measured metabolites in the cerebrospinal fluid.

Additional file 2: Table S2. Concentration levels of the measured metabolites in the plasma samples.

\section{Abbreviations \\ 5-HT: 5-hydroxy tryptamine (serotonin); AMPA: a-amino-3-hydroxy-5-methyl- 4-isoxazolepropionic acid; CFA: Complete Freund's adjuvant; CNS: Central nervous system; CGRP: Calcitonin gene-related peptide; CO: Control group; CSF: Cerebrospinal fluid; ECD: Electrochemical detector; FLD: Fluorescence detector; ssCX: Somatosensory cortex; GABA: $\gamma$-aminobutyric acid; GBD: Global Burden of Disease; Glu: Glutamate; HPLC: High performance liquid chromatography; IS: Internal standard; KYN: Kynurenine; KYNA: Kynurenic acid; LOD: Limit of detection; LOQ: Limit of quantitation; NA: Noradrenaline; $\mathrm{Na}_{2}$ EDTA: Disodium ethylenediaminetetraacetic acid NMDA: N-methyl-D-aspartate; PACAP: Pituitary adenylate cyclase-activating peptide; PCA: Perchloric acid; TNC: Trigeminal nucleus caudalis; TRP: Tryptophan; TS: Trigeminovascular system; TTH: Tension-type headache; UVD: Ultraviolet detector}

\section{Acknowledgements}

We would like to thank István Szatmári, from the Department of Pharmaceutical Chemistry, University of Szeged, for synthesizing 4hydroxyquinazoline-2-carboxylic acid.

\section{Authors' contributions}

EKCS, GV and NN designed the study, performed the HPLC experiments and analyzed the data and wrote the manuscript. TK, HP performed the animal experiments. As a corresponding author, ZD designed the study, wrote the manuscript and had final responsibility for the decision to submit for publication. JT, AP, KP, LV made further critical manuscript revisions. All authors read and commented on the final manuscript, furthermore approved the final version to be published.

\section{Authors' information}

Edina K Cseh and Gábor Veres contributed equally to this work.

\section{Funding}

The research was supported by GINOP-2.3.2-15-2016-00034 ('Molecular Biological Fundamentals of Neurodegenerative and Immune Diseases:
Therapeutic Trials with Kynurenines'), EFOP-3.6.1-16-2016-00008 ('Development of Intelligent Life Science Technologies, Methods, Applications and Development of Innovative Processes and Services Based on the Knowledge Base of Szeged'), 20391-3/2018/FEKUSTRAT (Ministry of Human Capacities, Hungary grant) and 2017-1.2.1-NKP-2017-00002 NAP VI/4 (Hungarian Brain Research Program grant). Dénes Zádori was supported by the János Bolyai Research Scholarship of the Hungarian Academy of Sciences and by the UNKP-18-4 New National Excellence Program of the Ministry of Human Capacities. Edina K Cseh and Tamás Körtési were supported by UNKP-19-3 New National Excellence Program of the Ministry for Innovation and Technology. Edina K Cseh was supported by the EFOP-3.6.3-VEKOP-16-2017-00009. Open access funding was provided by the University of Szeged (SZTE; grant number: 4576).

\section{Availability of data and materials}

The authors made available all of their data and materials on request.

\section{Ethics approval and consent to participate}

Our experiments complied fully with the guidelines of Act 1998/XXVIII of the Hungarian Parliament on Animal Experiments (243/1988) and with the recommendations of the International Association for the Study of Pain and European Communities Council (86/609/ECC). The studies were in harmony with the Ethical Codex of Animal Experiments and were approved by the Ethics Committee of the Faculty of Medicine, University of Szeged, with a permission number of XI./1102/2018.

\section{Consent for publication}

Not applicable.

\section{Competing interests}

The authors declare that they have no competing interests.

\section{Author details}

'Department of Neurology, Interdisciplinary Excellence Center, Faculty of Medicine, Albert Szent-Györgyi Clinical Center, University of Szeged, Semmelweis u. 6, Szeged H-6725, Hungary. ${ }^{2}$ MTA-SZTE Neuroscience Research Group, Szeged, Hungary.

Received: 17 January 2020 Accepted: 8 April 2020

Published online: 21 April 2020

\section{References}

1. Brennan KC, Pietrobon D (2018) A systems neuroscience approach to migraine. Neuron 97:1004-1021. https://doi.org/10.1016/j.neuron.2018.01.029

2. Noseda R, Burstein R (2013) Migraine pathophysiology: anatomy of the trigeminovascular pathway and associated neurological symptoms, CSD, sensitization and modulation of pain. Pain 154:44-53. https://doi.org/10. 1016/j.pain.2013.07.021

3. Harriott AM, Strother LC, Vila-Pueyo M, Holland PR (2019) Animal models of migraine and experimental techniques used to examine trigeminal sensory processing. J Headache Pain 20:91-106. https://doi.org/10.1186/s10194-0191043-7

4. Romero-Reyes M, Uyanik JM (2014) Orofacial pain management: current perspectives. J Pain Res 7:99-115. https://doi.org/10.2147/JPR.S37593

5. Tajti J, Párdutz A, Vámos E, Tuka B, Kuris A, Bohár Z et al (2011) Migraine is a neuronal disease. J Neural Transm (Vienna) 118:511-524. https://doi.org/10. 1007/s00702-010-0515-3

6. Aczél T, Kun J, Szőke É, Rauch T, Junttila S, Gyenesei A et al (2018) Transcriptional alterations in the trigeminal ganglia, nucleus and peripheral blood mononuclear cells in a rat orofacial pain model. Front Mol Neurosci 11:219-232. https://doi.org/10.3389/fnmol.2018.00219

7. Iwata K, Takeda M, Oh SB, Shinoda M. Neurophysiology of orofacial pain. In: Farah CS, Balasubramaniam R, McCullough MJ, editors. Contemporary Oral Medicine, Springer International Publishing; 2017. p. 1-23. doi: https://doi. org/10.1007/978-3-319-28100-1_8-1.

8. Lukács M, Haanes KA, Majláth Z, Tajti J, Vécsei L, Warfvinge K et al (2015) Dural administration of inflammatory soup or complete Freund's adjuvant induces activation and inflammatory response in the rat trigeminal ganglion. J Headache Pain 16:564-575. https://doi.org/10.1186/s10194-0150564-y 
9. Körtési T, Tuka B, Nyári A, Vécsei L, Tajti J (2019) The effect of orofacial complete Freund's adjuvant treatment on the expression of migrainerelated molecules. J Headache Pain 20:43-52. https://doi.org/10.1186/ s10194-019-0999-7

10. Kopach O, Viatchenko-Karpinski V, Belan P, Voitenko N (2012) Development of inflammation-induced hyperalgesia and allodynia is associated with the upregulation of extrasynaptic AMPA receptors in tonically firing lamina II dorsal horn neurons. Front Physiol 3:391-399. https://doi.org/10.3389/fphys. 2012.00391

11. Park JS, Yaster M, Guan X, Xu JT, Shih MH, Guan Y et al (2008) Role of spinal cord alpha-amino-3-hydroxy-5-methyl-4-isoxazolepropionic acid receptors in complete Freund's adjuvant-induced inflammatory pain. Mol Pain 4:67-78. https://doi.org/10.1186/1744-8069-4-67

12. Park JS, Voitenko N, Petralia RS, Guan X, Xu JT, Steinberg JP et al (2009) Persistent inflammation induces GluR2 internalization via NMDA receptortriggered PKC activation in dorsal horn neurons. J Neurosci 29:3206-3219. https://doi.org/10.1523/JNEUROSCI.4514-08.2009

13. Zhang B, Tao F, Liaw WJ, Bredt DS, Johns RA, Tao YX (2003) Effect of knock down of spinal cord PSD-93/chapsin-110 on persistent pain induced by complete Freund's adjuvant and peripheral nerve injury. Pain 106:187-196. https://doi.org/10.1016/j.pain.2003.08.003

14. Imbe H, Iwata K, Zhou QQ, Zou S, Dubner R, Ren K (2001) Orofacial deep and cutaneous tissue inflammation and trigeminal neuronal activation. Implications for persistent temporomandibular pain. Cells Tissues Organs 169:238-247. https://doi.org/10.1159/000047887

15. Okumura M, Iwata K, Yasuda K, Inoue K, Shinoda M, Honda K et al (2010) Alternation of gene expression in trigeminal ganglion neurons following complete Freund's adjuvant or capsaicin injection into the rat face. J Mol Neurosci 42:200-209. https://doi.org/10.1007/s12031-010-9348-7

16. Chung MK, Park J, Asgar J, Ro JY (2016) Transcriptome analysis of trigeminal ganglia following masseter muscle inflammation in rats. Mol Pain 12:1-12. https://doi.org/10.1177/1744806916668526

17. Puehler W, Rittner HL, Mousa SA, Brack A, Krause H, Stein C et al (2006) Interleukin-1 beta contributes to the upregulation of kappa opioid receptor mrna in dorsal root ganglia in response to peripheral inflammation. Neuroscience 141:989-998. https://doi.org/10.1016/j.neuroscience.2006.03. 078

18. Wu SX, Zhu M, Wang W, Wang YY, Li YQ, Yew DT (2001) Changes of the expression of 5-HT receptor subtype mRNAs in rat dorsal root ganglion by complete Freund's adjuvant-induced inflammation. Neurosci Lett 307:183186. https://doi.org/10.1016/S0304-3940(01)01946-2

19. Luo H, Cheng J, Han JS, Wan Y (2004) Change of vanilloid receptor 1 expression in dorsal root ganglion and spinal dorsal horn during inflammatory nociception induced by complete Freund's adjuvant in rats. Neuroreport 15:655-658. https://doi.org/10.1097/00001756-200403220-00016

20. Demartini C, Tassorelli C, Zanaboni AM, Tonsi G, Francesconi O, Nativi C et al (2017) The role of the transient receptor potential ankyrin type-1 (TRPA1) channel in migraine pain: evaluation in an animal model. J Headache Pain 18:94-103. https://doi.org/10.1186/s10194-017-0804-4

21. Takeda M, Tanimoto T, Kadoi J, Nasu M, Takahashi M, Kitagawa J et al (2007) Enhanced excitability of nociceptive trigeminal ganglion neurons by satellite glial cytokine following peripheral inflammation. Pain 129:155-166. https://doi.org/10.1016/j.pain.2006.10.007

22. Krzyzanowska A, Avendaño C (2012) Behavioral testing in rodent models of orofacial neuropathic and inflammatory pain. Brain Behav 2:678-697. https://doi.org/10.1002/brb3.85

23. Peres MFP, Zukerman E, Senne Soares CA, Alonso EO, Santos BFC, Faulhaber MHW (2004) Cerebrospinal fluid glutamate levels in chronic migraine. Cephalalgia 24:735-739. https://doi.org/10.1111/j.1468-2982.2004.00750.X

24. Martínez F, Castillo J, Rodríguez JR, Leira R, Noya M (1993) Neuroexcitatory amino acid levels in plasma and cerebrospinal fluid during migraine attacks. Cephalalgia 13:89-93. https://doi.org/10.1046/j.1468-2982.1993.1302089.x

25. Ferrari MD, Odink J, Bos KD, Malessy MJ, Bruyn GW (1990) Neuroexcitatory plasma amino acids are elevated in migraine. Neurology 40:1582-1586. https://doi.org/10.1212/WNL.40.10.1582

26. Cananzi AR, D'Andrea G, Perini F, Zamberlan F, Welch KM (1995) Platelet and plasma levels of glutamate and glutamine in migraine with and without aura. Cephalalgia 15:132-135. https://doi.org/10.1046/j.1468-2982. 1995.015002132.x

27. Campos F, Sobrino T, Pérez-Mato M, Rodríguez-Osorio X, Leira R, Blanco M et al (2013) Glutamate oxaloacetate transaminase: a new key in the dysregulation of glutamate in migraine patients. Cephalalgia 33:1148-1154. https://doi.org/10.1177/0333102413487444

28. Guerriero RM, Giza CC, Rotenberg A (2015) Glutamate and GABA imbalance following traumatic brain injury. Curr Neurol Neurosci Rep 15:27-38. https:// doi.org/10.1007/s11910-015-0545-1

29. Knyihár-Csillik E, Chadaide Z, Okuno E, Krisztin-Péva B, Toldi J, Varga C et al (2004) Kynurenine aminotransferase in the supratentorial dura mater of the rat: effect of stimulation of the trigeminal ganglion. Exp Neurol 186:242247. https://doi.org/10.1016/j.expneurol.2003.12.001

30. Vámos E, Párdutz Á, Varga H, Bohár Z, Tajti J, Fülöp F et al (2009) Lkynurenine combined with probenecid and the novel synthetic kynurenic acid derivative attenuate nitroglycerin-induced nNOS in the rat caudal trigeminal nucleus. Neuropharmacology 57:425-429. https://doi.org/10. 1016/j.neuropharm.2009.06.033

31. Vámos E, Fejes A, Koch J, Tajti J, Fülöp F, Toldi J et al (2010) Kynurenate derivative attenuates the nitroglycerin-induced CamKIla and CGRP expression changes. Headache 50:834-843. https://doi.org/10.1111/j.15264610.2009.01574.x

32. Chauvel V, Vamos E, Pardutz A, Vecsei L, Schoenen J, Multon S (2012) Effect of systemic kynurenine on cortical spreading depression and its modulation by sex hormones in rat. Exp Neurol 236:207-214. https://doi.org/10.1016/j. expneurol.2012.05.002

33. Párdutz Á, Fejes A, Bohár Z, Tar L, Toldi J, Vécsei L (2012) Kynurenines and headache. J Neural Transm 119:285-296. https://doi.org/10.1007/s00702011-0665-y

34. Körtési T, Tuka B, Tajti J, Bagoly T, Fülöp F, Helyes Z et al (2017) Kynurenic acid inhibits the electrical stimulation induced elevated pituitary Adenylate Cyclase-activating polypeptide expression in the TNC. Front Neurol 8:745. https://doi.org/10.3389/fneur.2017.00745

35. Zádori D, Klivényi P, Plangár I, Toldi J, Vécsei L (2011) Endogenous neuroprotection in chronic neurodegenerative disorders: with particular regard to the kynurenines. J Cell Mol Med 15:701-717. https://doi.org/10. 1111/j.1582-4934.2010.01237.x

36. Kessler M, Terramani T, Lynch G, Baudry M (1989) A glycine site associated with N-methyl-D-aspartic acid receptors: characterization and identification of a new class of antagonists. J Neurochem 52:1319-1328

37. Birch PJ, Grossman CJ, Hayes AG (1988) Kynurenate and FG9041 have both competitive and non-competitive antagonist actions at excitatory amino acid receptors. Eur J Pharmacol 151:313-315. https://doi.org/10.1016/00142999(88)90814-x

38. Deen $M$, Hansen HD, Hougaard A, Nørgaard M, Eiberg $H$, Lehel $S$ et al (2018) High brain serotonin levels in migraine between attacks: a 5-HT4 receptor binding PET study. Neuroimage Clin 18:97-102. https://doi.org/10. 1016/j.nicl.2018.01.016

39. Aggarwal M, Puri V, Puri S (2012) Serotonin and CGRP in migraine. Ann Neurosci 19:88-94. https://doi.org/10.5214/ans.0972.7531.12190210

40. Varga H, Párdutz A, Tajti J, Vécsei L, Schoenen J (2006) The modulatory effect of estrogen on the caudal trigeminal nucleus of the rat in an animal model of migraine. Ideggyogy Sz 59:389-395

41. Bussone $\mathrm{G}$ (2008) Cluster headache: from treatment to pathophysiology. Neurol Sci 29:1-6. https://doi.org/10.1007/s10072-008-0875-8

42. Benarroch EE (2018) Locus coeruleus. Cell Tissue Res 373:221-232. https:// doi.org/10.1007/s00441-017-2649-1

43. Prescott MJ, Lidster K (2017) Improving quality of science through better animal welfare: the NC3Rs strategy. Lab Anim (NY) 46:152-156. https://doi. org/10.1038/laban.1217

44. Cseh EK, Veres G, Szentirmai M, Nánási N, Szatmári I, Fülöp F et al (2019) HPLC method for the assessment of tryptophan metabolism utilizing separate internal standard for each detector. Anal Biochem 574:7-14. https://doi.org/10.1016/j.ab.2019.03.005

45. Nánási N, Hadady L, Cseh E, Veres G, Klivényi P, Vécsei L et al (2018) Development and validation of high performance liquid chromatography method for the measurements of biogenic amines. In: Tünde A, István I (eds) Proceedings of the 24th International Symposium on Analytical and Environmental Problems. University of Szeged, Szeged, pp 368-372

46. Veres G, Tellér A, Martos D, Szatmari I, Kiss L, Vécsei L et al (2019) Determination of glutamate and GABA from rat central nervous system samples with HPLC utilizing fluorescent detection. In: Tünde A, István I (eds) Proceedings of the 25th International Symposium on Analytical and Environmental Problems, pp 427-431 
47. Pawlak D, Tankiewicz A, Buczko W (2001) Kynurenine and its metabolites in the rat with experimental renal insufficiency. J Physiol Pharmacol 52:755-766

48. Ceresoli-Borroni G, Rassoulpour A, Wu HQ, Guidetti P, Schwarcz R (2006) Chronic neuroleptic treatment reduces endogenous kynurenic acid levels in rat brain. J Neural Transm (Vienna) 113:1355-1365. https://doi.org/10.1007/ s00702-005-0432-z

49. Kucharewicz I, Kasacka I, Pawlak D, Tankiewicz-Kwedlo A, Mroczko B, Buczko W et al (2008) The concentration of kynurenine in rat model of asthma. Folia Histochem Cytobiol 46:199-203. https://doi.org/10.2478/v10042-0080030-7

50. Sultana N, Arayne MS, Khan MM, Saleem DM, Mirza AZ (2012) Determination of tryptophan in raw materials, rat brain and human plasma by RP-HPLC technique. J Chromatogr Sci 50:531-537. https://doi.org/10. 1093/chromsci/bms045

51. Zagajewski J, Drozdowicz D, Brzozowska I, Hubalewska-Mazgaj M, Stelmaszynska T, Laidler PM et al (2012) Conversion L-tryptophan to melatonin in the gastrointestinal tract: the new high performance liquid chromatography method enabling simultaneous determination of six metabolites of L-tryptophan by native fluorescence and UV-VIS detection. J Physiol Pharmacol 63:613-621

52. Samavati R, Zádor F, Szúcs E, Tuka B, Martos D, Veres G et al (2017) Kynurenic acid and its analogue can alter the opioid receptor G-protein signaling after acute treatment via NMDA receptor in rat cortex and striatum. J Neurol Sci 376:63-70. https://doi.org/10.1016/j.jns.2017.02.053

53. Wu H-Q, Guidetti P, Goodman JH, Varasi M, Ceresoli-Borroni G, Speciale C et al (2000) Kynurenergic manipulations influence excitatory synaptic function and excitotoxic vulnerability in the rat hippocampus in vivo. Neuroscience 97:243-251. https://doi.org/10.1016/S0306-4522(00)00030-0

54. Saylor D, Steiner TJ (2018) The global burden of headache. Semin Neurol 38:182-190. https://doi.org/10.1055/s-0038-1646946

55. Abraham J, Ackerman I, Aggarwal R, Ahn SY, Ali MK, Alvarado M et al (2012) Years lived with disability (YLDs) for 1160 sequelae of 289 diseases and injuries 1990-2010: a systematic analysis for the global burden of disease study 2010. Lancet 380:2163-2196. https://doi.org/10.1016/S01406736(12)61729-2

56. GBD 2015 DALYs and HALE Collaborators (2016) Global, regional, and national disability-adjusted life-years (DALYs) for 315 diseases and injuries and healthy life expectancy (HALE), 1990-2015: a systematic analysis for the Global Burden of Disease Study 2015. Lancet 388:1603-1658. https://doi. org/10.1016/S0140-6736(16)31460-X

57. Schuster NM, Rapoport AM (2016) New strategies for the treatment and prevention of primary headache disorders. Nat Rev Neurol 12:635-650. https://doi.org/10.1038/nrneurol.2016.143

58. American Headache Society (2019) The American headache society position statement on integrating new migraine treatments into clinical practice. Headache 59:1-18. https://doi.org/10.1111/head.13456

59. Diener HC, Charles A, Goadsby PJ, Holle D (2015) New therapeutic approaches for the prevention and treatment of migraine. Lancet Neurol 14:1010-1022. https://doi.org/10.1016/S1474-4422(15)00198-2

60. Obermann M, Holle D, Naegel S, Burmeister J, Diener HC (2015) Pharmacotherapy options for cluster headache. Expert Opin Pharmacother 16:1177-1184. https://doi.org/10.1517/14656566.2015.1040392

61. Sarchielli P, Di Filippo M, Nardi K, Calabresi P (2007) Sensitization, glutamate, and the link between migraine and fibromyalgia. Curr Pain Headache Rep 11:343-351. https://doi.org/10.1007/s11916-007-0216-2

62. Bereiter DA, Benetti AP (1996) Excitatory amino release within spinal trigeminal nucleus after mustard oil injection into the temporomandibular joint region of the rat. Pain 67:451-459. https://doi.org/10.1016/03043959(96)03156-9

63. Lukács M, Warfvinge K, Tajti J, Fülöp F, Toldi J, Vécsei L et al (2017) Topical dura mater application of CFA induces enhanced expression of c-fos and glutamate in rat trigeminal nucleus caudalis: attenuated by KYNA derivate (SZR72). J Headache Pain 18:39-46. https://doi.org/10.1186/s10194-0170746-X

64. Oshinsky ML, Luo J (2006) Neurochemistry of trigeminal activation in an animal model of migraine. Headache 46:39-44

65. Osikowicz M, Mika J, Przewlocka B (2013) The glutamatergic system as a target for neuropathic pain relief. Exp Physiol 98:372-384. https://doi.org/10. 1113/expphysiol.2012.069922

66. Bleakman D, Alt A, Nisenbaum ES (2006) Glutamate receptors and pain. Semin Cell Dev Biol 17:592-604. https://doi.org/10.1016/j.semcdb.2006.10.008
67. Eide K, Stubhaug A, Oye I, Breivik H (1995) Continuous subcutaneous administration of the $\mathrm{N}$-methyl-D-aspartic acid (NMDA) receptor antagonist ketamine in the treatment of post-herpetic neuralgia. Pain 61:221-228. https://doi.org/10.1016/0304-3959(94)00182-e

68. Jevtovic-Todorovic V, Wozniak DF, Powell S, Nardi A, Olney JW (1998) Clonidine potentiates the neuropathic pain-relieving action of MK-801 while preventing its neurotoxic and hyperactivity side effects. Brain Res 781:202211. https://doi.org/10.1016/s0006-8993(97)01247-x

69. Afridi SK, Giffin NJ, Kaube H, Goadsby PJ (2013) A randomized controlled trial of intranasal ketamine in migraine with prolonged aura. Neurology 80 : 642-647. https://doi.org/10.1212/WNL.0b013e3182824e66

70. Alt A, Weiss B, Ogden AM, Li X, Gleason SD, Calligaro DO et al (2006) In vitro and in vivo studies in rats with LY293558 suggest AMPA/kainate receptor blockade as a novel potential mechanism for the therapeutic treatment of anxiety disorders. Psychopharmacology 185:240-247. https:// doi.org/10.1007/s00213-005-0292-0

71. Sang CN, Ramadan NM, Wallihan RG, Chappell AS, Freitag FG, Smith TR et al (2004) LY293558, a novel AMPA/GluR5 antagonist, is efficacious and welltolerated in acute migraine. Cephalalgia 24:596-602. https://doi.org/10.1111/ j.1468-2982.2004.00723.x

72. Curto M, Lionetto L, Negro A, Capi M, Fazio F, Giamberardino MA et al (2015) Altered kynurenine pathway metabolites in serum of chronic migraine patients. J Headache Pain 17:47. https://doi.org/10.1186/s10194-016-0638-5

73. Curto M, Lionetto L, Negro A, Capi M, Perugino F, Fazio F et al (2015) Altered serum levels of kynurenine metabolites in patients affected by cluster headache. J Headache Pain 17:27. https://doi.org/10.1186/s10194016-0620-2

74. Schwarcz R (2004) The kynurenine pathway of tryptophan degradation as a drug target. Curr Opin Pharmacol 4:12-17. https://doi.org/10.1016/j.coph. 2003.10.006

75. Zádori D, llisz I, Klivényi P, Szatmári I, Fülöp F, Toldi J et al (2011) Timecourse of kynurenic acid concentration in mouse serum following the administration of a novel kynurenic acid analog. J Pharm Biomed Anal 55: 540-543. https://doi.org/10.1016/j.jpba.2011.02.014

76. Vécsei L, Szalárdy L, Fülöp F, Toldi J (2013) Kynurenines in the CNS: recent advances and new questions. Nat Rev Drug Discov 12:64-82. https://doi. org/10.1038/nrd3793

77. Szalardy L, Zadori D, Toldi J, Fulop F, Klivenyi P, Vecsei L (2012) Manipulating kynurenic acid levels in the brain - on the edge between neuroprotection and cognitive dysfunction. Curr Top Med Chem 12:17971806. https://doi.org/10.2174/156802612803989264

78. Bohár Z, Párdutz Á, Vécsei L (2016) Tryptophan catabolites and migraine. Curr Pharm Des 22:1013-1021

79. Vámos E (2012) Protective compounds in animal models of trigeminal activation and neurodegeneration. Ideggyogy Sz 65:64-70

80. Messlinger K, Russo AF (2019) Current understanding of trigeminal ganglion structure and function in headache. Cephalalgia 39:1661-1674. https://doi. org/10.1177/0333102418786261

81. Tuboly G, Tar L, Bohar Z, Safrany-Fark A, Petrovszki Z, Kekesi G et al (2015) The inimitable kynurenic acid: the roles of different ionotropic receptors in the action of kynurenic acid at a spinal level. Brain Res Bull 112:52-60. https://doi.org/10.1016/j.brainresbull.2015.02.001

82. Knyihar-Csillik E, Mihaly A, Krisztin-Peva B, Robotka H, Szatmari I, Fulop F et al (2008) The kynurenate analog SZR-72 prevents the nitroglycerolinduced increase of c-fos immunoreactivity in the rat caudal trigeminal nucleus: comparative studies of the effects of SZR-72 and kynurenic acid. Neurosci Res 61:429-432. https://doi.org/10.1016/j.neures.2008.04.009

83. Park MK, Lee JH, Yang GY, Won KA, Kim MJ, Park YY et al (2011) Peripheral administration of NR2 antagonists attenuates orofacial formalin-induced nociceptive behavior in rats. Prog Neuro-Psychopharmacol Biol Psychiatry 35:982-986. https://doi.org/10.1016/j.pnpbp.2011.01.018

84. Veres G, Fejes-Szabó A, Zádori D, Nagy-Grócz G, László AM, Bajtai A et al (2017) A comparative assessment of two kynurenic acid analogs in the formalin model of trigeminal activation: a behavioral, immunohistochemical and pharmacokinetic study. J Neural Transm (Vienna) 124:99-112. https:// doi.org/10.1007/s00702-016-1615-5

85. Fejes-Szabó A, Bohár Z, Vámos E, Nagy-Grócz G, Tar L, Veres G et al (2014) Pre-treatment with new kynurenic acid amide dose-dependently prevents the nitroglycerine-induced neuronal activation and sensitization in cervical part of trigemino-cervical complex. J Neural Transm (Vienna) 121:725-738. https://doi.org/10.1007/s00702-013-1146-2 
86. Noseda R, Borsook D, Burstein R (2017) Neuropeptides and neurotransmitters that modulate thalamo-cortical pathways relevant to migraine headache. Headache 57(Suppl 2):97-111. https:/doi.org/10.1111/head.13083

87. Castle D, Robertson NP (2018) Monoclonal antibodies for migraine: an update. J Neurol 265:1491-1492. https://doi.org/10.1007/s00415-018-8886-8

88. Bigal ME, Walter S, Rapoport AM (2015) Therapeutic antibodies against CGRP or its receptor. Br J Clin Pharmacol 79:886-895. https://doi.org/10. 1111/bcp.12591

89. Raffaelli B, Reuter U (2018) The biology of monoclonal antibodies: focus on calcitonin gene-related peptide for prophylactic migraine therapy. Neurotherapeutics 15:324-335. https://doi.org/10.1007/s13311-018-0622-7

90. Vollesen AL, Benemei S, Cortese F, Labastida-Ramírez A, Marchese F, Pellesi L et al (2018) Migraine and cluster headache - the common link. J Headache Pain 19. https://doi.org/10.1186/s10194-018-0909-4

\section{Publisher's Note}

Springer Nature remains neutral with regard to jurisdictional claims in published maps and institutional affiliations.

Ready to submit your research? Choose BMC and benefit from:

- fast, convenient online submission

- thorough peer review by experienced researchers in your field

- rapid publication on acceptance

- support for research data, including large and complex data types

- gold Open Access which fosters wider collaboration and increased citations

- maximum visibility for your research: over $100 \mathrm{M}$ website views per year

At $\mathrm{BMC}$, research is always in progress.

Learn more biomedcentral.com/submissions 


\section{Neurotransmitter and tryptophan metabolite concentration changes in the Complete Freund's adjuvant model of orofacial pain}

Edina K Cseh ${ }^{1, \#}$, Gábor Veres ${ }^{1,2, \#}$, Tamás Körtési ${ }^{1}$, Helga Polyák ${ }^{1}$, Nikolett Nánási ${ }^{1}$, János

Tajti $^{1}$, Árpád Párdutz ${ }^{1}$, Péter Klivényi ${ }^{1}$, László Vécsei ${ }^{1,2}$, Dénes Zádori ${ }^{1 *}$

${ }^{1}$ Department of Neurology, Interdisciplinary Excellence Center, Faculty of Medicine, Albert

Szent-Györgyi Clinical Center, University of Szeged, Szeged, Hungary;

${ }^{2}$ MTA-SZTE Neuroscience Research Group, Szeged, Hungary

\#These authors contributed equally to this work

Table S1 Concentration levels of the measured metabolites in the cerebrospinal fluid

\begin{tabular}{cccc}
\hline & $\begin{array}{c}\text { Control group } \\
(\mathrm{n}=5)\end{array}$ & $\begin{array}{c}\text { CFA 24 h } \\
(\mathrm{n}=5)\end{array}$ & $\begin{array}{c}\text { CFA 48 h } \\
(\mathrm{n}=4)\end{array}$ \\
\hline Glu & \multicolumn{2}{c}{ Cerebrospinal fluid } \\
$(\mu \mathrm{M})$ & 6.08 & 9.87 & 8.61 \\
$\mathrm{GABA}$ & $6.04-9.60$ & $4.90-16.5$ & $6.13-9.96$ \\
$(\mu \mathrm{M})$ & 1.38 & 1.35 & 1.54 \\
$\mathrm{TRP}$ & $1.04-1.49$ & $1.23-2.10$ & $1.40-1.73$ \\
$(\mu \mathrm{M})$ & 1.40 & 1.32 & 1.55 \\
$\mathrm{KYN}$ & $0.96-1.60$ & $1.24-3.77$ & $1.13-1.95$ \\
$(\mu \mathrm{M})$ & $<\mathrm{LOD}$ & 0.21 & $<\mathrm{LOD}$ \\
$\mathrm{KYNA}$ & 3.57 & $0.13-0.22$ & 4.10 \\
$(\mathrm{nM})$ & $1.61-11.0$ & 3.29 & $3.14-6.32$ \\
\hline
\end{tabular}

Results are shown as median $\left(1^{\text {st }}-3^{\text {rd }}\right.$ quartile). $C F A$ Complete Freund's adjuvant, $G A B A$ gamma-aminobutyric acid, Glu glutamate, $K Y N$ kynurenine, $K Y N A$ kynurenic acid, $n$ number of animals per group, TRP tryptophan 


\section{Neurotransmitter and tryptophan metabolite concentration changes in the Complete Freund's adjuvant model of orofacial pain}

Edina K Cseh ${ }^{1, \#}$, Gábor Veres ${ }^{1,2, \#}$, Tamás Körtési ${ }^{1}$, Helga Polyák ${ }^{1}$, Nikolett Nánási ${ }^{1}$, János

Tajti $^{1}$, Árpád Párdutz ${ }^{1}$, Péter Klivényi ${ }^{1}$, László Vécsei ${ }^{1,2}$, Dénes Zádori ${ }^{1 *}$

${ }^{1}$ Department of Neurology, Interdisciplinary Excellence Center, Faculty of Medicine, Albert

Szent-Györgyi Clinical Center, University of Szeged, Szeged, Hungary;

${ }^{2}$ MTA-SZTE Neuroscience Research Group, Szeged, Hungary

\#These authors contributed equally to this work

Table S2 Concentration levels of the measured metabolites in the plasma samples

\begin{tabular}{cccc}
\hline & $\begin{array}{c}\text { Control group } \\
(\mathrm{n}=9)\end{array}$ & $\begin{array}{c}\text { CFA 24 h } \\
(\mathrm{n}=9)\end{array}$ & $\begin{array}{c}\text { CFA 48 h } \\
(\mathrm{n}=8)\end{array}$ \\
\hline TRP & 63.9 & 81.4 & 56.4 \\
$(\mu \mathrm{M})$ & $(52.4-78.2)$ & $(54.3-88.1)$ & $(51.6-76.1)$ \\
$\mathrm{KYN}$ & 4.58 & 4.72 & 3.27 \\
$(\mu \mathrm{M})$ & $(3.29-4.98)$ & $(4.45-5.12)$ & $(2.83-4.79)$ \\
$\mathrm{KYNA}$ & 129 & 172 & 139 \\
$(\mathrm{nM})$ & $(120-184)$ & $(99.9-214)$ & $(95.0-173)$
\end{tabular}

Results are shown as median $\left(1^{\text {st }}-3^{\text {rd }}\right.$ quartile). CFA Complete Freund's adjuvant, $K Y N$ kynurenine, KYNA kynurenic acid, $n$ number of animals per group, TRP tryptophan 